QK203.8

. C3:

Carter, William R

A preliminary catalogue of the flora of Vancouver and. Queen Charlotte Islands 


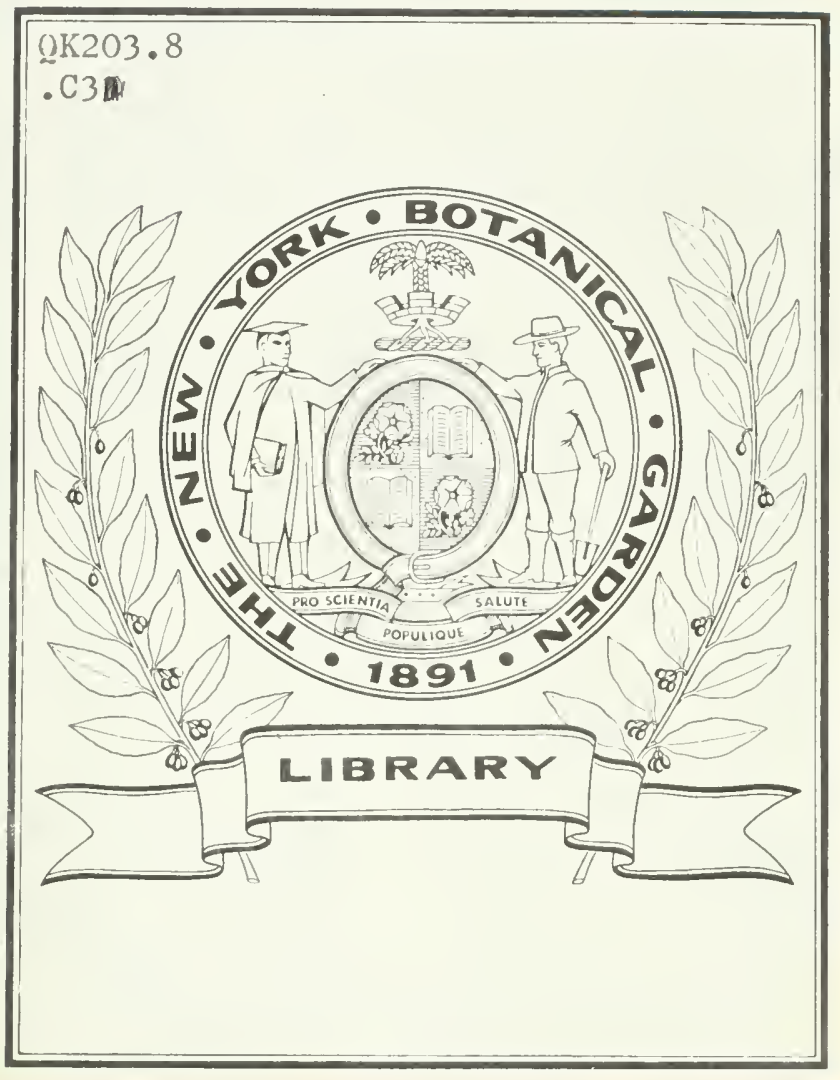




PROVINCIAL MUSEUM OF. NATURAL HISTORY VICTORIA, B.C.

yi

\section{A PRELIMINARY CATALOGUE}

OF THE

\section{Flora of Vancouver and Queen Charlotte Islands}

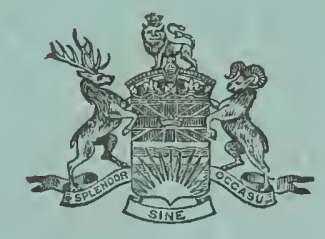





\section{PROVINCIAL MUSEUM OF NATURAL HISTORY}

VICTORIA, B.C.

出

\section{A PRELIMINARY CATALOGUE}

OF THE

Flora of Vancouver and Queen Charlotte Islands

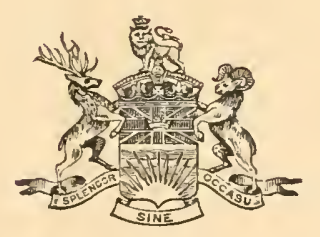

VICTORIA, B.C.:

Priuted by William H. Cullin, Printer to the King's Most Excellent Majesty. 1921. 

Propixcial Mesecal of Nateral History.

Victoria, B.C., March 7 th, 1921.

The Honomrable .I. D. JacLean, M.D.,

Prorincial secretary and Minister of Education.

Tictoria, B.C.

Sir.-I hare the honour, as Inirector of the Provincial Museum of Natural History, to submit a preliminary Catalogue of the Flora of Vanconver and gueen Charlotte Islands.

I have the honour to be,

Sir,

Your obedient servant,

FR.ANCIS FERMODE,

Director. 



\section{CATALOGUE}

\section{OF THE \\ Flora of Vancouver and Queen Charlotte Islands.}

\section{INTRODUCTION.}

A Floril is a botanical account of the plants of a country or district. with the orders or families systematically arranged moler the chasses, the genera under the orders, and the species (when there are more than one) under the genus to which they belong.

Of late years there has been a great awakening in the study of the flowering plants of this rovince; many incmiries are made as to the names of the more popular plants and as to where their descriptions can be fonme. To meet these demands the Provincial Muselim undertook to add greatly to the Herbarium, and receired unbounded support from many persons who were rery much interested in this branch of natural listory. More aud more people are beginning to realize the pleasure and profit that can be gained from the observation of the plants growing around us, observing their beats, and acruiring a knowledge which greatly increases an appreciation of nature.

Botanical biology teaches us the study of plants cammot be overlooked. The rely existence of the animal kingdom is solely dependent on regetable life; the great problems of geographical astribution; the theory of organic evolution or the immermost secrets of vital phenomena, whether in health or disease, are simplified hy the study of plants. Botany and zoology are dependent on each other, without one the other would cease to exist. It is a study which cannot be indefinitely postponed. as the old terrestrial order is fast passing awar before our eres; everywhere the primitive regetation is disalpearing as more and more of the earth's surface is brought under cultivation and our forests are denuted of their original growth. Much work has been done, and still greater is the work which has set to be accomplished.

This list will. withont donbt. give some idea of the many species which are to be found on Vancouver and Queen Charlotte Islands. wiving known locations in which they have been collected. The scientific or Latin name is given first, followed by the English or popular name, those plints that have been introduced being printed in italic letter's.

liecognizing the works and writings of our earliest botanists and biologists. the nomenclature is according to Griç's Manual wherever possible, and many other morks have been consulted in comnection with on westem species. such as the "Floria Borcali-Americana," by Hooker" "Flora of the North-west Coast," by Piper and Beattie; "Illustrated Flora of the Northeru States and Canada." by Britton and Brown; "Flora of Southern British Columlia." by J. K. Henry ; "Flora of the Rocky Mountains and Adjacent I'lains," ly I'. A. liyduerg. Ph.I), ; and other botanical works.

The late Mr. J. M. Macom amounced in the early part of the year 1913 that he had the manuscript for" a "Flora of Vancourer Island" almost ready for the lress, ${ }^{*}$ and that it would embody the results of several years" work by himself, Professor Macoun, and Mr. Spreadborough in various parts of Vancouver and the Queen Charlotte Islands. For reasous of ill-health and cramped quarter's after

* Ottawa Naturalist. Xxvi., Feb.. 1913. 
the fire at the Parlianent Buildings at Ottawa, his intentions were not fulfilled, although he often s]roke of the urgent necessity of such a work.

After the presentation to the Provincial Musem of the collection of Vanconver Island plants made br Irofessor Macoun, of which partial lists have been [rinted in our Ammul Rejorts, * both father and son pointed ont the desirability of publisbing a catalogue of the flowering plants of Vanconver Island and the Queen Charlotte Group, to be based upon Professor Macoun's "Catalogue of Canadian Ilants" and on Mr. J. M. Macom's notes on new species and additions as published from time to time in the "Canadian Record of Science" and the "Ottawa Naturalist."

They promised full co-operation in order to revise the old nomenclature and bring it into conformity with the usage of the Gray Ilerbarium of IJarvard Lniversity, In institution from which they had always had most valuable assistance in cases of doubtful identity of species.

MI. J. M. Macoun also undertook to supply full information as to unrecorded species collected during the last few years, so that the catalogue might he as full as possible.

Soon aftel the mork was under way the world lecame the poorer by the loss of both of the Macoms. It will be impossible, therefore, to make such a complete list as anticipated, and the compilers are fully aware that it must contain many errors both of omission and commission.

In spite of this, it is hoped that it may prove to be useful as a basis for future worker's to huild oul.

The bulls of this information las been compiled in the Department by Mr. W. R. Carter, who las done considerable collecting on Vancouver Island. He has been greatly assisted by $\mathrm{Dr}$. C. F. Tewcombe, who has supplied a great amount of infolmation in regard to the Flora of both Vancouver and the Queen Charlotte Islands, as his comnection with anthropological research in the Province has carried him to many points, especially on the west coast, that would not probably be visited by the ordinary botanist. Dr. Newcombe also put at the disposal of the Department his rare botanical library which has been the means of supplying much data and intormation not otherwise obtainable.

The thanks of the Defartment are also due, for information, records, and slecimens, to Dr. C. F. Newcombe; the late Professor Macom; the late J. M. Macoun, C.M.G., Chief of the Biological Division of the Geological Survey, Ottawa; Mr. W. B. Anderson; Mr. J. R. Anterson: I'rofessor J. K. Henry; Mr. W. A. Newcombe; and others, whose kindness is gratefully acknowledged. .

F. K.

Victoria, B.C., Warch $\approx t h, 1921$.

*Pr. Mus. Rep. for 1915, 1916, 1917, 1918. 


\section{POLYPODIACEZE (Fern Family).}

Adaxter (Maidenhair).

1. pedatum $\mathrm{L}$.

Generally dlistrilntel, Queen Charlotte Islands; Vancouver Island.

A. ledatmm aleuticum Rupr.

Locally distributed, Mount Lienson, V.I., Macoun.

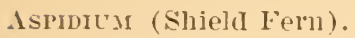

1. oreopteris Sw. (Mountain sinield Fern.)

rale. Shawnitan Latke. V.I., J.R. Anderson.

A. ristam Hoffum. (Rigris shield Fern.)

Mount Finlayson, Y.I.

A. spinulosum Swartz. (Nas'row Prickly-toothed Feru.)

Common. Vaneonver Island.

rar. (lilatatum (Hoffu.) Ilook. (Broad Prickly-toothed Ferm.)

Common. Queen Charlotte Islands; Vancouver Island.

Asplexily (Spleenwort).

A. Filix-femina (L.) Bermh. (Lady Fern.)

Common. Skidegate, Queen Charlotte Islands; Vanconver Island.

A. Trichomanes L. (Maidenhair Spleenwort.)

Generally distributed, Queen Charlotte Islands; Vanconver Island.

A. viride Huds. (Green sileenwort.)

Rale. Queen Charlote Islands, Dr. C. F. Tcueombe; Lchelet, V.I.

Cheilaxthes (Lip Feln).

C. gracillima I. C. Waton. (Lace Fern.)

Locally distributed, southern lalf of Vancouver Island-Cowichan; Mount Finlayson.

Cryptograma (Irock Brake).

C. acrostichoides Ii. Br. (I'arsley Fern.)

Common. Vanconver Island.

C. densa (Brack.) Diels. (Clift Brake.)

Locally distributed, sontleln half of Vanconver Island-Alberni; Sproat I.ake; Mount Finlayson.

Crstopteris (Bladder Feln).

C. fragilis L. (Brittle Bladder Fern.)

Generally distributed, Queen Charlotte Islands: Vancourer Island.

C. montana Bernh. (Mountain Bladder Ferm.)

Cowichan, V.I. (Glendemuing's List, 1918.)

GYMNOGRAMME.

G. triangularis Kaulf. (Silver-back Fel'n.)

Locally distributed, southern end of Vanconvel Island-Namaino; Mount Finlayson; Victoria.

Phegopteris (Beech Feru).

I'. alpestris (Hoppe.) Mett. (Alpine Beech Feru.)

Rare. Stratheona Park, V.I., J. M. Macoun.

I'. Dryopteris (I.) Fee. (Oak Fel'n.)

Generally distributed, Queen Charlotte Islands; Vancourer Island. 
PIIEgOPTERIS-Contillicel.

P. polypodioides Fee. (Common Beech Fern.)

Rare. Queen Cluarlotte Islands, C. DeB. Green. Strathcona Park, Y.I., J. M. Macomn.

Polspoduar (Polspody).

P. rulgare L. (Common Polypody; Licorice-root Fern.)

var. orcillentale IIook.

Common. Vancouver Island.

P. Scoulel' Hook, \& Gray, (Scouler's Polypody.)

Locally distributed. west coast of Vanconver Island; Queen Charlotte Islands; Triangle Island.

P. hesperimu Maxon.

Locally distributed, Qneen C'harlotte Islauds: Vancourer Island.

Polystichuar.

P. Andersonii Hopkins. (Anderson Shield Fern.)

Rare. Stratheona Park, V.I., IT. B. Anderson.

I'. Brannii (Spenner) Fee. (Prickly Shield Fern.)

Locally distributed, Nootka, V.I.

P. Lonchitis (L.) Roth. (Holly Fern.)

Generally distributed in momtains, Vanconver Islaud.

P. munitum (Kaulf.) Presl. (Chamisso's Shield Fern.)

Common. Queen C'harlotte Islands; Vanconver Island.

P. munitum var. imblicans (D. C. Eaton) Maxon.

Generally distributed, Tancourer Island.

P. munitum inciso-serratum (D. C. Eaton) Maxon.

Locally distribnted, Vanconver Island near Victoria.

P. scopulinum (D. C. Eaton) Maxon.

Texada Island. II. B. Anderson.

Pteris (Bracken).

P. aquilina L. (Pracken.)

Common. Queen Charlotte Islands; Vanconver Island.

\section{STRUTHIOPTERIS.}

S. spicant (L.) Weiss. (Deer Ferm.)

Common. Tancourer Islaud; Queen Charlotte Islands.

WOODSIA.

IV. oregana D. C. Eaton. (Oregon Woodsia.)

Rare. Cowichan River, V.I., Macomn.

W. scopulina 1). C. Eaton. (Rocky Mountain Woodsia.)

Locally distributed, sonthern half of Vancourer Island-Victoria: Sooke.

Woodwamda (Chain Feru).

W. spinulosa Mart. \& (ral. (Great Cluain Fern.)

Iiale. 'Texada Island, IT. B. Audersom; Saanich Inlet, V.I.

\section{OPHIOGLOSSACEZE (Adder's Tongue Family).}

Botrychium (Grilje leru; Moomwort).

B. virginianum (I.) S. W. (Rattlesnake Fern.)

Vancouver Island-Alberni; Cowichan.

B. silaifolium I'resl. (Leathery Grape Feru.)

Common. Vancouver Islaud.

B. simplex F. Hitch. (I.ittle (irape Fern.)

Iare. Momnt Ibenson, V.I., Macomn. 
EqLiSETU.

\section{EQUISETACE无 (Horsetail Family).}

E. arvense L. (Common IIolsetail.)

Generally distributed, Queen C'larlotte Islands: Vincouver lsband.

E. hyemile L. (S.ouring Rush.)

Locally (Iistributed, Vancourer Island-Goldstrean: Victoria.

E. limosmu L. (Swimu) Horsetail.)

Lorally distributed, west coist of Vancoures Island-Barkley Sound, Jucoun.

E. litorile Künl. (Slıore IIorsetail.)

Generally distributed, Vanconver Island.

E. palustre L. Vill. polystaclibum Hook. (Marsh Horsetail.)

Locally distributed, Fanconver Island-Shawnigan. Queen Charlotte lskinds.

E. ramosissimmm Desf.

Locally distributed, Shawnigan Lake, V.I., Maroun.

E. telmateia Ehrh. (Great Horsetail.)

Locally distributed, Vancourex Island-Sidney ; Deadman Iojer.

E. vilriegatum sirhleich.

Locally distributed, Vancouver Island-Horne Iake; Camelon Lake; Shawnigan Ialke, Wueoun.

\section{LYCOPODIACE $\mathbb{E}$ (Club Moss Family).}

Jacoponuar (Club Moss).

L. ammotimum L. vilr. pungens sipring. (Stiff ('lul, Moss.)

Locally distributed, Vancouver Island-Mount Arrowsmith. Queen Charlotte Islands.

L. clavatum L. (Common ('lub; Stagholu Moss.)

Common. Queen Charlotte Islinds; Vancouver Island.

L. complanatum L. (Ground Cedar.)

Locally distributed, Vaneourer Island-Mount Mark; Mount Arrowsmith, yacoull.

L. immdatum L. (Marsh Club Moss.)

Locally distributed, Ccluelet, V.I., Mucoun.

L. lucidulum Michx. (Nhining Clul, Moss.)

Locally distributed. Vanconvel Island-Cowichan Lake; Mayne Island.

L. Seligo L. (Fir ('lub Moss.)

Locally distributed, skidegate, Queen Charlotte Islands, I)r. r. Tr. Tr.

combe. Vanconrel Island-Nount Benson, Mucomn; Cowichall Iatie.

L. sitchense Rupr. (Alaskan ('lub Moss.)

Locally distributed, Vancouver Island-Mount Arowsmith.

\section{SELAGINELLACEÆ.}

SElaglatella.

S. densa RṬlb.

Locally distributed, Tancouver Island-Tcluelet.

S. Wallacei Hieron.

Vanconver Island-Victoria : Malahat; Alberni; Cowichan.

S. mupestris (L.) Suring. (Iock Selagrinella.)

Geuerally distributed, Queen Charlotte Islands; Vancouver.

S. selaginoides (L.) Link. (Low Selaginella.)

Locally distributed, Celuelet, Y.I., Macomn.

S. struthioloides (Presl.) Underw.

Locally distributed, west coast of Vancourer Island. First collected at Nootka Sound. 


\section{ISOETACEÆE (Quillwort Family).}

Isoetes (Quillwort).

I. echinospora rar. Braunii (Durieu) Engelm. (Braun's Quillwort.)

Locally distributed, Great Central Lake, V.I., W. R. Carter.

I. maritima Underw.

Locally distributed, Salt Marshes, Alberui, V.I., Macumn.

I. Nuttallii A. Br. (Nuttall's Quillwort.)

Locally distributed, Vancomer Island-Alberni; Wellington.

TAXT'S.

\section{TAXACE $\mathbb{E}$ (Yew Family).}

'T. brevifolia Nutt. (Yew.)

Generally distributed, Queen Charlotte Islands; Vancourer Islaud.

\section{PINACEæ (Pine Family).}

ABIES.

1. amabilis (Lond.) Forbes. (Lorely Fir.)

Generally distributed in mountains, Vancouver Island.

A. grandis Lindl. (White Fir.)

Generally distributed, Vancourer Island.

CilaM.ecYParis (Cypless).

C. nootkatensis (Lamb.) Spach. (Yellow or Alaska Cerłar.)

Locally distributed, Queen Cluarlotte Islands. Vancourer Island-Mount Arrowsuith; Port Renfrew; Clayoquot; and northern end of Vancouver Island.

JUNiperus (Jumiper).

J. communis rar. montana Ait.

Generally distributed, Queen Charlotte Islands; Vancourer Island.

J. scopulorum Sarg.

Locally distributed, Vancourer Island, on the islands and mainland about the Gulf of Georgia, and northern part of Puget Sound; not elsewhere known west of Cascade Mountains.

PICEA (Spruce).

P. sitchensis (Bong.) Trautr. \& Meyer. (Sitka Spruce.)

Generally distributed, Queen Charlotte Islands; Vanconver Island.

Prixus (Pine).

I'. contorta Dougl. (Scrub Pine; Lodge-pole Pine.)

Generally distributed, Queen Charlotte Islands; Vancouver Island.

P. monticola Dougl. (Westeru White Pine.)

Locally distributed, Vancouver Island-Goldstream; Cameron Lake;

Alberni; Henderson Lake.

PSEunotsiga.

P. mucromata (IRaf.) Sudw. (Donglas Fir.)

Common. Vancourer Island, with exception of the north end.

THuJA (Arbor Vitio). (c'edar.)

T. plicata Donn. (Giant Cedar.)

Common. Qneen Clarlotte Islands; Vancouver Island. 
Tstaid (Hemlock).

T. Leterophylla (Raf.) Sarg. (Western llemlock.)

(ommon. Queen Cliarlotte Islands; Vancouver Island,

Y. mertensiani (Bongr) ('ar'. (Mountain 1Iemlock.)

Generally distributed in the mountains, Qucen Cluarlotte Islands; Van(courer Island.

\section{TYPHACEÆ (Cat-tail Family).}

Tร̈на.

T. latifolia L. (Cat-tail.)

Generilly distributed, Vancourer Island.

\section{SPARGANIACEEE (Bur-reed Family).}

SPARGAXIUM.

S. americanum var. androcladum (Englm.) F. \& E. (Branching Bur-leed.) Generally distributed, sonthern half of Vancouver Island.

S. angustifolium Michx. (Narrow-leaved Lur-reed.)

Local!y distributed, Vancourer Island-Shamigan Lake.

S. (ireenei Morong.

Vanconver Island. (Henry's "Flora of Souther'n British Columbia,")

S. minimm Fries. (Small Bur-reed,)

Rare. Renfrew District, Rosendahl of Brand; Vancourer Island.

S. simplex Huds. (Simple-stemmed Bur-reed.)

Generally distribnted, Vancouver Island.

LIL.モA.

\section{NAJADACEAE (Pondweed Family).}

L. subulata HBK.

Rare. Alberni, V.I., Macoun; Hcmry.

NAJAS ( Naiad).

న. flexilis (W'illd.) Rostk. \& Schmidt.

Locally distributed, southern half of Vancouver Island-Sproat Lake and Jalies near Victoria.

Phyllospanix (False Eel Grass).

P. Scouleri Hook.

Locally distributed, Tancourer Island-Icluelet; Sidney; Victoria.

P. Torreyi Wats.

Locally distributed. Tancourer Island (Spreadborongh) ; Lcluelet, Macoun.

On rocky shores, Vancouver Island to California.

Potaliogeton (Pondweed).

P. alpinus Balbis.

Locally distributed, Vanconver Island-Nanaimo.

P. americauns C. \& S.

Somenos Lake, V.I. (Gleudeming"s List, 1918.)

P. epihylutus Raf.

Quamichan Lake, V.I. (Glendenning's List, 191S.)

P. filiformis Pers.

Courtenay, V.I., Macoun.

I. heterophyllus Schreb. (Various-leaved Pondweed.)

Generally distributed, Vancouver Island. 
Potanogeton-Continned.

P. natans I. (Common Floating Pondweed.) Generally distributed. Vancouver Island.

P. pectinatus L. (Fennel-leared Pondweed.)

Generally distributed, Vaucouver Island, Macom.

P. prielongus Wult.

Locally distributed, sonther'n end of Vancouver Island-Lost Lake; Langford lake; Shaw

P. pusillus L. (Small Pondweed.)

Generally distributed, Vanconver Island.

P. Richardsunil (Bemn.) Ryolb.

Quanichan Lake, V.I.

P. Robbinsii Oalies.

Locally distributed, southern half of Vancouver Island-Somass River; Alberni ; Lost Lake.

P. zosterifolius Schum.

Locally distributed. Vancouver Island-Langford Lalie, Macoun; Bearel Lake; Cowichan Lake.

Ruppla (Ditch Grissi).

R. maritima L.

Tanconver Island-Alberni; Yictoria.

Zostera (Eel Grass; ('rab Grass; Sea Wrack).

Z. marina I.

Generilly distributed. Queen C'Larlotte Islands; Vancouver Island.

Z. latifolia Morong.

Locally distributed. Tancourer 1sland-Esquimalt, Macomn.

\section{JUNCAGINACEEE (Arrow Grass Family).}

SCHEUCHZERTA

S. palustris L.

Iille. Cowichan Lake, T.I.

Triglochix (Arrow Grass).

T. maritimal L. (Seat-side Arrow Griss.)

Common. Queen ('harlotte Islands; Vancourer Island.

T. palustris L. (Marsh Arrow Grass.)

Queen Charlotte Islands; Mar'ses, Vancourer Island-Cameron Lalie.

\section{ALISMACE王 (Water-plantain Family).}

Alisira (Water-plantain).

A. Plantago-arputica L.

Very locally distributed, Vancourer Island-Courtenas; Cowichan.

Sagittaria (Alrow-lieid).

S. latifolia Willd. (Proad-leaved Alrow-head.)

Liare. Vanconver Island-Wellington, Wr. R. Carter.

\section{GRAMINE民 (Grass Family).}

Aira (Hair Grissi).

A. caryophyll'u L. (Silvery IJair Grass.)

Common. Queen Charlotte Islands; Vancouver Island.

A. precerer I. (Early Hair Grass.)

Cominon. Vilncourer Islaud. 
Agropyrox (Wheat Griss).

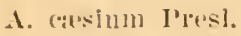

Iinle. Villeouver Islaurl-Yictoria.

1. divergens Nees.

Locially distributed, 'Lexitlal Is]and, Jourson; Vanconver Island.

1. oceidentale serib.

Vinconver Islancl. (Henry"s " I'loria of Sontheln British Columbia.")

1. repens (J.) Benur. (couclu Grass)

(imenelly distributed, Vancourel Island.

A. tenerum Vasey. (Slender Wheat Grass.)

L.ecally distributed, Vancouver Island-Nanaimo; Goldstream; Duncan;

victoria.

Agliostis (Bent Grass).

1. reguivalis Trin.

Locally distributed. Qneen Clarlotte Islands. Vancourel Island-Renfrew (Iosendilhl) : Toluelet : Vijotoria.

A. allua L. (IRed Top.)

Common. Vancouver Island.

var. milritima (Lam.) Meyer.

Locally distributed, Triangle Is]and, Dr. r. F. Teucrumb; Long Beach, V.I.

A. (anina I. (Brown Pent Glass.)

Common. Vancouver Island.

A. exaratil $\mathrm{x}$ alba Trin.

Locally distributed. Vanconver Island-Comox; Sidney. Texada Isiand.

A. slomerata (Presl.) Kinnth.

Rare along the sea-const of Tancouver Island to Northern California.

I'robalbly only a sub-splecies of (A. exalrata Trin.) (sre Piper \& Beattie's

"Floril of the Nortliwest (oast.")

A. hyemilis (Walt.) BSI'. (Rough Hair Grass.)

Locally distributed, Vanconver Island-Sidney. Texada Island.

A. inflata Scroibuer.

Locally distributed, Vanconver Island-Espuimalt.

1. longiligula Hitch.

Locally distributerI. Vancouver Island-Departure Bay; Bear Lake.

A. micropliylla Stend.

Locilly distributed, sonther'n half of Vancourel Island-Qualicum;

Naniluo: (owiclan; Victoria.

A. oregonensis Vaser.

Locally distributed, Vanconver Island-Renfrew (Rosendahl); rare at

Ecluelet.

A. stolonifera L.

Common. Vauconver Island.

var. malritima Lam.

Common. Vancouver Islank.

1. varians Trin.

Gneen Charlotte Islands, Dursou. (Macomn's "Catalogur of Plants.")

1. vulgaris $\mathrm{L}$.

Iocally Jistributer. Tancourer Island-Ÿictoria.

Alopectrus (Foxtail Grass).

A. aristulatus Moc. (Slunt-awed Foxtail.)

Vanconver Island-IBeaver Lake; Elk Lalie.

A. californicus Vasey.

Locally distributed, Vancouver Island-Head of Lcinelet Inlet. 
ALOPECLRUS-C'outinued.

A. geniculatus L. (Marsh Foxtail.)

Locally distributed, Vanconver Island-Sidney.

A. matensis L. (Meadow Foxtail.)

Locally distributed, Vancourer Island-Nanaimo; Victoria; Cowichan.

A. saceatus Vasey.

Vancouver Island. (Henry"s "Flora of Southern British Columbia.")

Axthoxantion (Sweet Ternal Grass).

A. odoratum L.

Generally distributed, Vancourer Island.

Arrexatherum (Oat Grass).

A. clatius (I.) Beauv.

Locally distributed, southern end of Vancouver Island-Beacon Hill Park.

rictoria; Cadbora Bay (Momnt Douglas. "Cedir Hill").

Avena (Oat).

1. fatur var. glabiata Peter. (Wild Oat.)

Generally distributed. Vanconver Island.

A. sativa I. (Cultivated Oat.)

common. Vancouver Island.

A. strigosa Schreb.

Locally distributed, Vanconvel Island-Sooke, Macoun.

Beckanaña (Tall slough Grass).

B. erucatormis (J.) IIost. Var. uniflora Scrib.

Locally distributed, Vancourer Island-Victoria, Macomn

BrizA (Quaking Grass).

B. mediu $\mathbf{L}$.

Rare. Nanaimo, V.I., Maconn; Alberni, V.I., W. R. carter.

Bromus (Brome Grass).

B. aleutensis Triu.

Locally distributed, Queen Charlotte Islands. (Osgood, "North American Fiuna," No. 21.)

B. carinatus H. \& 1 .

Locally distributed, Vaucouver lslaud-Cowichan.

B. commutatus Schrad.

Locally distributed. Tancouver Island-Cowichan Lake; Victoria.

B. eximius (Sliear.) Pijer.

Incilly distributed, Vancourer Island-Alberni.

B. horleacers I. (Soft Cliess.)

Locally tistributed, Vancourer Island-Sidney.

B. inermis Leyss. (Hungarian Brome.)

Locally distributed, Vaucourer Island-Cowichan.

B. Macomili Vasey.

Iocally distributel, Vanconver Islaud-Nanaimo; Victoria.

I3. Hal'orinatus Nees.

Iforlly distributed, Vancourer Island-Nanaimo.

li. marimlls lest.

Ifrally distributed, Vancouver Island-Sidney; Victoria.

I. Oreuttianus Vasey.

Locally distributed, sonthern end of Vancouver Island-Victoria. 
Bromes-C'ontinnerl.

I3. pacificus shear.

Locally distributed. Vancourer Island-Sidney, Macoun.

13. racemosus I. (Smootli Brone Grass.)

Locally distributed, Vancouver Island-Alberni; Sidney.

I. rubr'ns I.

Ioeally distributed, Vancourer Islant-Nanaimo.

B. scculinus I. (Chess.)

Generally distributed, southern half of Vancouver lsland-Alberni;

victoria.

I3. sitchensis Pong.

Locally distribnted. Vancourer Islaud-Siduey, Mucoun.

B. stcrilis L. (Barren Brome Grass.)

Locally distributed, Vancourer Island-Saanichton; Victoria.

13. tectorlum I. (Downy Brome Grass.)

Locally distributed, Tancourer Island-Saanichton, Macoun.

B. vulgaris Shear.

Generally distributed, sonthern half of Vancouver Island-Comox: Victoria.

b. vulgaris robustus Shear.

Generally distributed, sonthern half of Vanconver Island-Comox; Alberni ;

Cowichan.

\section{(alamagrostis (Reed Bent Grass).}

('. aleutica Trin.

Locally distributed, Triangle Island, $D r$. C. F. Icucombs. Fancourer

Island-Aberni; Victorja. Queen Charlotte Islands, Dawson.

(. canadensis var. acuminata Vasey. (Blue-joint Grass.)

Locally distributed, Vanconver Is]and-Comox; Departure Bay.

C. classiglumis 'liurb.

Locally distributet. Nimkish Lake, Dauson: Horne Lake, V.I. Mucoun.

c. hyperborea Lange. (Northern Reed Grass.)

Lowally distribnted, Tanconver Island-Tenfrew District, Roscnduhl of

Branel.

C. inexpansal Gray. (IBog Reed Grass.)

Vancouver Island-C'ameron Lake.

C. Langsdorffii (Link.) Trin.

Locally distributed, Queen Charlotte Islands, Douson. Fancourer Island-

Jount Arrowsmith; Cameron Lake.

C. stricta Beaur.

Locally distributed near Sooke, V.I., Damson; Nootka; Obserratory Inlet.

Crxya (Indian Reed Grass).

C. latifolia (Trev.) Griseb.

Locally distributed, Vanconver Island-Qualicum; Comox; Alberni; Nanaimo.

C'ronox ( Rermuda Grass).

C. Ductylon (L.) P'ers.

Iacally distributed, Vancomrer Island-Nanaimo; Victoria.

Crxosures (Dog's-tail Grass).

C. cristutus $\mathrm{L}$.

Generally distributed, Vanconver Island.

C. echinatus $\mathrm{L}$.

Locally distributed, Vanconver Island-Xanaimo; Victoria. 
Dactrus (Orchard Grass).

D. glomeratu L.

Common. Queen Charlotte Islands; Yancouver Island.

Dantionia (Wild Oat Grass).

D. americina Scribn.

Generally distributed, southern half of Vancouver Island-Alberni; Cowicluan ; Victoria.

D. californica Boland.

Generally distributed, sonthern half of vanconver Island-Alberni; Nanaimo; Sidney.

D. intermedial Vasey.

Locally distributed in mountains, Vanconver Island-Mount Arrowsmith.

D. pinetorim Piper.

Locally distributerl, Vancourer Island-Mount Benson.

D, unispicata Thurb. (AIountain Oat Grass.)

Tancourer Island. (Macoun's "Catalogue of Plants.")

Deschampsia (Tall Hair Grass).

D. atropur]urea (Wallu.) Scheele. (Monntain Hair Grass.)

Locally distributed, Vancouver Island-Mount Benson; Stratheona Park.

D. caespitosal (L.) Lieaur. (Tufted Hair Grass.)

Common and very variable. Queen Charlotte Islands; Vanconver Island.

D. cespitosa var, arctica.

Ginlf of Georgia and Queen Charlotte Islands, Dauson.

D. calycina P'resl.

Locally distributed, Vanconver Island—Victoria; Nanaimo.

D. elongata (Hook.) Munro.

Generally distributed, southern half of Vancouver Island-Alberni;

Victoriil.

Distichlis (Alkali; Salt Grass).

D. spicata (L.) Greene.

Common. Salt marshes of Vancouver Island.

Echinochloa (Barnyard Grass).

E. crus-gulli (Limn.) Beaur.

Locally distributed, Vancouver Island-Experimental Farm, Sidney, Iucoun.

Elyus (Wild Rive).

L. americanus Vasey \& Scribner.

('ommon, Yancouver Island. (Macoun's " Catalogue of I'lants.")

E. arenarius mollis l'iper.

Locally distributed, Queen CIrarlotte Islands. Vincourer Island-Nootka ;

Silluer:

E. arenatius var. villosus F. Mey.

Locilly distributed, Valleourer Island-Alberni.

E. borealis scrilut.

Locally distributed, Vanconver Island-Ienfrew, Roscnduhl; Ccluelet;

Alberni : Sillney.

E. condensatus Iresl.

Locally distributerl, Vanconver Is]anr.

E. hirsutus I'resl.

Iocally distrihuted, Vamouver Islamd-Tear Lalke; Cowichan Lake; Silney. 
Eliuts-f'ontinued.

E. glaucus Buckl. (Smooth Wild Rye.)

Common. Tancourer Island.

var. lobustus Dily.

Locally distributed, Vancouver Island-Lcluelet; Victoria.

E. vancouverensis Vasey.

Locally distributed, southern half of Vancouver Island-Comox; Sidney;

Victoria. Bare Island.

Festica (Fescue Grass).

F. brevifolia Watson.

Iiare. Mount Arrowsmith, Y.I., Marvmu.

var. polyphrila Vases.

Locally distributed, Vaucouver Island.

F. Uromoides $\mathrm{L}$.

Locally distributed, Vancouver Island-Alberni.

$F$. clatior L. (Tall Meadow Fescue.)

Locally distributed, Yancouver Island-Comox; Cowichan. Mayne Island, Iacoun.

F. idahoense Elmer.

Localls distributed, Mayne Island, Macoun.

F. megalura Nutt.

Locally distributed, southern half of Vancourer Island-Sidney; Comox.

Mayne Island.

F. Myuros Linn. (Rat's-tail Fescue Grass.)

Generally distributed, Vancourer Island.

F. occidentalis Hook. (Western Fescue Grass.)

Locally distributed, Vancouver Island-Cowichan; Sidney.

F. octoflora Walt. (Sleuder Fescue Grass.)

Locally distributed, Vancourer Fsland-Vanaimo, Macoun.

$F$. orina L. (Sheep's Fescue Grass.)

Generally distributed, Tancouver Island; Queen Charlotte Islands, Dauson.

F. pacifica Piper.

Common. Vancourer Island-Nanaimo; Victolia.

F. reflexa Bucli].

Tancourer Island. (Henry"s "Flora of Southeru British Columbia.")

F. rubra L. (Red Fescue Grass.)

Common. Queen Charlotte Islands; Triangle Island; Princess Royal

Island: Tancouver Island.

F. subulata Triu.

Locally distributed, Vancouver Island-Bear Lake; Nanaimo; Sidney;

Fenfrew. Queen Charlotte Islands.

F. subuliflora Scribn.

Locally distributed, Yancouver Island-Comox; Sidney.

Glyceria (Manna Grass).

G. borealis (Nash.) Batch.

Locally distributed. Yancourer Islaud-Cowichan; Beaver Lake.

G. canadensis (Michx.) Trin.

Locally distributed, Vanconrer Island-Somenos Lake, Macoun.

G. grandis Wats.

Generally distributed, southern half of Tancouver Island.

G. lejtostachya Buckl.

Rare. Alberni, V.I., Henry. 


\section{GLYCERIA-Continued.}

G. nervata (Willd.) var. elata Nash. (Fowl Meadow Grass.)

Vancouver Islaud-Alberni; Nanaimo.

G. pumila Vasey.

Rare. Barkley Sound, V.I., Mfacoun.

G. pauciflora Presl.

Locally distributed, southern half of Vaneouver Island-Nootka; Victoria; Cowichan.

G. septentrionalis Hitch.

Locally distributed, Vancouver Island-Cowichan; Victoria. (The western form of G. fluitans (L.) R. Br.)

\section{Hierochlä̈ (Holy Grass).}

H. odorata (L.) Wahlenb.

Locally distributed, southern half of Vanconver Island-Alberni; Comox; Cowichan.

Hor.cus (Velvet Grass).

H. lanatus $\mathrm{L}$.

Common. Triangle Island; Vancourer Island.

Hordeum (Barley).

II. boreale Scrib. \& Smith.

Rare. Renfrew District, V.I., Rosendahl.

H. Gussoneanum Parl.

Locally distributed, Vancouver Island-Victoria, Macoun.

II. jubatum L. (Squirrel-tailed Grass.)

Locally distributed, Vancouver Island-Cowichan.

$H$. maritimum With.

Locally distributed, Vancouver Island-Nanaimo, Macoum.

M. murinum L. (Wall Barley.)

Locally distributed, Vancouver Island-Nanaimo; Victoria.

H. nodosum L. (Meadow Barley.)

Generally distributed, Vancouver Island.

H. pusillum Nutt. (Little Barley.)

Locally distributed, Vancouver Island-Victoria.

Kócleria (Shining Spike Grass).

K. cristata (L.) Pers.

Generally distributed, southern half of Vancouver Island.

Lolium (Darnel; Rye Grass).

L. multiflorum Lam. (Italian Rye Grass.)

Common. Vancouver Island.

L. perenne L. (Rye Grass.)

Common. Yancouver Island.

L. temulentum (L.) Darnel. (Poison Darnel.)

Locally distributed, Vancouver Island-Duncin; Victoria.

Melica (Melic Grass).

I. Geyeri Mun'o.

Rare. Alberui, V.I., II enry.

M. Harfordii Boland.

Rather rare (Macoun). Vancourer Island-Alberni; Sidney. 
Merica-Continuca.

II. Smithii (Porter) Vasey.

liare. Alberni, V.I., IIcury.

M. subulata (Griseb.) seribn.

Vancouver lsland-Albelni; Cameron Liake.

JIBORA,

1. rerna Beaur.

Rare. Experimental Farm, Sidney, V.I., Macoun.

Paxicum (Panic Grias).

P. agrostoides spreng. (Red-top Panic.)

Iocally distributed, Vancouver Island-Sproat Lake, Macoun.

P. barbipulvinatum Nash. (Barbed Witch Grass.)

Locally distributed, Vancouver Island.

P. capillare L. (Witch Grass.)

Locally distributed, Vinconver Island-Stamp Falls, Alberni, Macoun;

Victoria,

P. pacificum Hitche. \& Chase.

Locally distributed. Vancouver Island-Renfrew district, Rosendahl; Bear

Lake; Strathcona Park, J. M. Macoun.

P. occidentale Scribn.

Locally distributed, Vancouver Island-Nootka; Alberni.

P. Scribnerianum Nash.

Locally distributed, Vancouver Islaud-Cowichan; Alberni; Horne Lake.

P. unciphyllum Trin.

On rocky beaches, crevices of slate rocks, Renfrew District, Roscndaht \& Butters.

Phalaris (Canary Grass).

P. arundinacea L. (Reed Camary Grass.)

Locally distributed, Vancouver Island-Courtenay River; Ash River;

Somenos Lake.

P. canaricusis L. Iocally distributed, Vancourer Island-Outer Wharf, Victoria, Macoun.

P. minor Retz.

Locally distributed, Vancouver Island-Namaimo, Macoun.

Phleum ('Timothy).

P. alpinum L. (Mountain Timothy.)

Generally distributed in mountains, Vancouver Island.

P. matense L. (Timothy.)

Common. Vancourer Island.

Phragaites (Tall leed grass).

P. communis Trin.

Vilncouver Island-Elk Lake.

Pos (Meadow Grass; Spear Grass).

P. alpina $\mathrm{L}$.

Generally distributed in mountaius, Vancouver Island-Mount Benson; Iount Arrowsmith.

P. annua L. (Low Spear Grass.)

Generally distributed. Vancouver Island; Dawson Harbour, Q.C.I., with erery indication of being a native plant. 
PoA-Continuce.

I'. Bolanderi Vasey.

Locally distributed, Vancourer Island-Sooke.

P. compressa L. (Canada Blue Grass.)

Locally distributed, Vancouver Island-Alberni ; Sidnes.

P. confinis Vasey.

Locally distributed in sand on sea-shore, Vancouver Island-Qualicum;

Cadboro Bay; Comox.

P. eminens Presl.

Locally distributed, Tancourer Island-Long Beach, J. R. Audcrson.

(Additions to "Flora of Vancouver Island," J. M. Macoun.)

P. Howellii Vasey.

Locally distributed, Vancouver Island-Goldstream; Sidney.

P. macrantha Vasey.

Locally distributed, Vancouver Island-Victoria.

P. neradensis Vasey.

Locally distributed, southern end of Vanconver Island-Cowichan.

P. nerrosa (IIook.) Vasey.

Locally distributed, Tancouver Island-Nootka, Scouler.

P. paddensis Williams.

Rare. Mount Arrowsmith, V.I., Mucoum; Strathcona Park, V.I., J. M. Mucoun.

P. pratensis L.

Common. Vancouver Island; Triangle Island.

P. Sandbergii Vasey.

Locally distributed. Vancouver Island-Sidnes.

P. trifiora Gilib. (False Red-top.)

Locally distributed, Triangle Island. Southern half of Vancourer Island-

Sidney; Cowichan; Renfrew. Queen Charlotte Islands.

P. tricialis L. var. filiculmis Scribner. "(Rough Meadow Grass.)

Locally distributed, Comox, V.I., Hecoun.

Polypogon (Beard Grass).

P. littoralis (With.) Smith.

Locally distributed, Vancouver Island-Victoria.

Puccinelia (Spear Grass).

P. angustati (12. Br.) Rand \& Redfield. (Arctic Meadow-Grass.)

Localls distributed in salt marshes on the coast of Tancouver Island.

I'. (listans (L.) I'arl. (Sweet Grass.)

Locally distributed, Queen Charlotte Islands. (Osgood, "North American

Fauna," No. 21.)

P. festucueformis (Host.) Parl.

Victoria, Oak Bay, V.I.

P. Lemmoni (Vasey) Scribn.

Locally distributed, coast of Vancouver Island.

SECAIE (Rye).

S. cercalc L.

Generilly distributed. (Escaped from cultivation.)

Setaria (Bristly Fox-tail Grass).

S. Italica (L.) lbeauv. (Millet.) (Escaped from cultivation.)

Locally distributed, Fancouver Island-Nlberni; Cowichan.

s. rivillis (L.) Ineauv. (Green Fox-tail Grass.)

Iocally Ristributed, Fancourer Island-Sidney. 
Stipl (Feather Grass).

S. comata 'Triu. \& Iiupr. (Needle Grass.)

Locally distributed, Vancourer Island-Victoria.

S. Lemmoni Seribu.

Locally distributed, Vilncourer Island. (Mount Douglas, "Cerlar Hill.")

S. viridula Trin. (Feather Bunch Grass.)

Locally distributed, southern end of Tancouver Island-Cowichan; Victoria.

Trisetum (False Oat).

T. canescens Buckl.

Loeally distributed, southern half of Vancourer Island-Horne Lake;

Nanaimo; Sooke; IIount Douglas ("Cedar Hill").

T. cernuum 'Trin.

Geuerally distributed, Vaucouver Island; Princess Royal Island; Banks

Island; Queen Cliarlotte Islauds.

T. spicatum (L.) Richter. (Narrow False Oat.)

Locally distributed in mountains, Vancouver Island--Mount Mark; Mount

Arrowsmith.

Tritices (Wheat).

T. vulgare I. (Cultivated Wheat.)

\section{CYPERACE无 (Sedge Family).}

CAREX (Sedge).

C. ablata Bailey.

Locally distributed. Tancourer Island-Mount Mark, Macoun.

C. abdita Bicknell.

Victoria. V.I. (Henry's "Flora of Southern British Columbia.")

C. acuta L. var. prolixa Hornem.

Locally distributed, Vancouver Island-Namaimo; Shawnigan Lake; Qual-

icum; Mount Douglas ("Cedar Hill").

C. acutina Bailey.

Locally distributed, Tancourer Island-Conichan River. (Glendenuiug's List, 1918.)

('. ienea Femald. (Fermald's Hay Secke.)

Locally distributed, Vancourer Island-Departure Bay; Alberui.

C. alpina Srrartz.

Queen Charlotte Lslands, Dauson.

C. atloxantha Presl.

Locally distributed, Queen Charlotte Islands. (Henry's "Flora of Soutlern British Columbia.")

C. aquatilis Wahl.

Locally distributed, Vancouver Island-Lost Lake, Macoun.

C. arcta Boott. (Northern-clustered Sedge.)

Common. rancouver Islaud.

C. anthrostachya Olnes.

Locally distributed, Vancouver Island-Nanaimo; Sidney. Macoun.

C. atrata Linn.

Queen Charlotte Islands, Dauson.

C. aurea Nutt. (Golden-fruited Sedge.)

Locally distributed, southeru half of Vancouver Island-Horne Lake;

rictoria.

C. bicolor All.

Locally distributed, Vancouver Island-Alberui; Shawnigan. 
Carex-Continucd.

C. Bolanderi Olney.

Generally distributed, Tancourer Island.

vax. Deweyana Schwein.

Generally distributed, Vancouver Island.

C. brevicaulis Mackenzie.

Locilly distributed, Vancouver Island-Elk Lake, Macoun.

C. brunnescens (Pers.) Poir.

Locally distributed, Vanconver Island-Cowichan. (Glendenning's List, 1918.)

C. canescens L. (IIoary Sedge.)

Locally distributed, Vancouver Island-Courtenay; Horne Lake; Alberni. C. circinata Meyer.

Tasu and Dawson Harbour, Queen Charlotte Islands.

C. cryptocarpa C. A. Meyer.

Common. Vanconver Island; Queen Charlotte Islands, Dauson.

C. densa Bailey.

Locally distributed, Vanconver Island-Nootka.

C. dives Holn.

Generally distributed, Vancouver Island.

C. exsiceata Bailey.

Generally distributed, Vancouver Island.

C. flava rectirostrata Bailey.

Locally distributed, Vancouver Island-Elk Lake, Macoun.

C. flcxilis Rudge.

Locally distributed, Vancouver Island-Celnelet. Introduced from the East with Cramberry plants.

C. feta Bailey.

Alberni, V.I., IIcmiy.

C. filiformis L. (Slender Sedge.)

Horne Lake, V.I., Macoun; Cowichan, V.I. (Glendenning's List, 191S.) (C. lasiocarpa Ehrh.)

C. globosa Boott.

Bear Lake, Cowichan, V.I.

C. Goodenowii Gay.

Queen Charlotte Islands. (Henry"s "Flora of Southern British Columbia.")

C. Hendersoni Bailey.

Generally distributed, Vanconver Island.

C. Hindsii Clarke.

Locally distributed. Vancouver Island. "(Pijer \& Beattie's "Flora of the Northwest Coast.")

C. illota Iailey.

Strathcona Park, Y.I., J. M. Macoun.

C. interrupta Boeckl.

Generally distributed, Vancouver Island.

C. invisa Bailey.

Iount Arrowsmith, V.I.; Princess Royal Island.

C. Ielloggii Boott.

Generally distributed, Vancouver Island.

C. laeviculmis Meinsch.

Locally distributed, Vanconver Island-Mount Mark, Macoun.

C. lanuginosa Michx.

Locally distributed, lakes, Vanconver Island, (Henry"s "Flora of Southern British Columbia.") 
CAREX-Continucl.

C. lejocurpa C. A. Meyer.

- Skidegate, Q.C.I., Dr. C. I. Neveombe.

C. Jeprrina L. (Hare's-foot Sedge.)

Locally distributed, Vanconver Island-Sidney; Alberui; Inut Arrowsmitl.

C. Ieptalea Wahl. (Bristle-stalked Sedge.)

Generally distributed, Vancourer Island-Nomnt Mark; Nanaimo; Malahat; Sidney.

C. Ifeersii Willd. (Little Prickly Sedge.)

Locally distributed, Vancouver Island-Teluelet; Wellington; Cowichan; Alberni.

C. Liddonii Boott.

Mount Douglas ("Cedar Hill"), T.I., Maroun.

C. limosa L.

Comox, V.I. ; Mount Mark, Y.I., Macoun.

C. livida (Wahlenb.) Willd. (Livid Sedge.)

Locally distributed, Tancouver Island-Renfrew District, Roscudahl of Brand.

C. macrocephala Willd.

Locally distributed on sandy sea-shore, Vancourer Island; Sarary Island; Queen Charlotte Islands.

C. macrochreta C. A. Meser.

Skidegate, Q.C.I.

C. magnifica Dewer.

Generally distributed, Yancouver Island; Tasu Harbour, Q.C.I.

C. Mertensii Prescott.

In mountains, Vancouver Island-Nount Mark; Mount Arrowsmith.

C. Novæ-Anglixe Schwein.

Mount Arrowsuith, Y.I., Macoun.

C. nigricans C. A. Meyer.

In mountains, Vanconver Island-Mount Arrowsuith.

C. obmupta Bailey.

Alberni, Y.I., Henry.

C. Ederi Retz. (Green Sedge.)

Generally distributed, margins of gravelly lakr-shores, Vancouver Island.

C. pachystachya Cham.

Generally distributed, Vancouver Island.

c. pauciflora Lightf. (Few-flowered Sedge.)

Langford Lake, V.I.

C. pampercula Michx.

Locally distributed, Vancourer Island-Mount Mark, Muromm. Ninstints,

Q.C.I. Specimen examined by Professor C. V. Pipel probubly this species.

C. phreocephala Piper.

Locally distributed, Vancouver Island-Comox: Silney. Mayne Island, Macoun.

C. physocarpa Presl.

Nootka, Kémedy Lilke, Y.I.

C. Pineri Mackenzie.

Sidney, V.I.. Macoun.

C. polygama Schkr.

Locally distributed, forme Lake, T.I.

C. praticola Rydb.

Locally distributed, Alberui, V.I., Henry. 


\section{Carex-Contimued.}

C. Preslii Steud.

Generally distributed, Vancouver Island.

C. pyrenaica Wabl.

In mountaius, Vancouver Island-Mount Arrowsmith.

C. rigida Good.

Vancouver Island. (Henry"s "Flora of Southern British Columbia.")

C. Rossii Boott.

Generally distributed, Vancourer Island.

C. rostrata Stokes. (Beaked Sedge.)

Generally distributed, Vancouver Island.

C. salina Wahl, var. robusta Bailey.

Locally distributed, Qualicum River, V.I.

C. scirpoides Michx. (Scirpus-like Sedge.)

Mount Arrowsmith, T.I., Macoun.

C. scoparia Schk.

Ueluelet, V.I. Introduced from the East with Cranberry plants.

C. sitchensis Prescott.

Generally distributed, Vancourer Island.

C. stygia Fries.

Canoe Pass, Skidegate, Q.C.I.

C. stellulata (var.) ?

Locally distributed, southeru half of Vancourer Is]and-Alberni; Mount

Mark; Goldstream; Mount Newton. Yakoun Lake, Q.C.I.

C. tenuirostris Olney.

Locally distributed, Vancouver Island-Comox; Victoria.

C. teretiuseula ampla var. ramosa Boott.

Generally distributed, southern half of Vancouver Island-Alberui ; Qualicum ; Victoria.

c. Tolmiei Boott.

Cowichan River, V.I. (Glendenning's List, 1918.)

c. utriculata Boott.

Generally distributed, Vauconver Island.

(. respertiua (Bailey) Howell.

Generally distributed, Yancouver Islaud.

C. Vesicaria var. major Boott.

Near Victoria. Wellington; Qualicum; sproat Lake. V.I.

Crperus (Galingale).

C. inflexus IInhl.

Rare. Alberni, V.I., Macoun.

\section{Dulichium.}

D. arundinaceum (L.) Brit.

Vancouver Islaud-Sproat Lake; Elk Lake.

Eleociraris (Spike Iiush).

E. acicularis (I.) R. \& S. (Ieast Spike Rush.)

Locally distributer, Alberui, Vancourer Island.

E. obtusa (Willd.) Schult. (Blunt Spike Rush.)

Locally distributed, Vancouver Island-Cameron Lake.

E. palustris (L.) Ii. \& S. (Creeping Spike Rush.)

Common. Vancouver Island.

E. rostellata 'Torr. (Beaked spike Rush.)

Locally distributed, silt marshes of Vancouver Island-Alberui, Macoun. 
Eriophorua (Cotton Griss).

E. Chamissonis C. A. Mey. (Russet Cotton Grass.)

Generally distributed, Queen Charlotte Islands; Vancouver Island.

E. polrstachyon L. (Broad-leaved Cotton Grass.)

Generally distributed, Queen Charlotte Islauds; Vanconver Island.

RrNchosponA.

R. alba (L.) Vahl. (White-topped Bog Rush.)

Locally distributed, Vancouver Island-Sproat Lake; Horne Lake, Macuin;

Reufrew District.

Scirpus (Club Rush; Bulrush).

S. americanus Pers. (Chair-maker's Rush.)

Generally distributed, Vaucouver Island.

S. ciespitosus Linu. (Tufted Club Rush.)

Locally distributed, Mount Arrowsmith. V.I., Macoun.

S. microcarpus Presl. (Small-fruited Bulrush.)

Generally distributed, Vancouver Island.

S. nanus Spreng.

Locally distributed, Vancourer Island-Alberni.

S. occidentalis (Wats.) Chase. (Viscid Great Bulrush.)

Locally distributed, Vancourer Islaud-Sidney, Muroun.

S. paucitlorus Lightf.

Locally distributed, Queen Charlotte Islands (Osgood, "North American

Fauna," No. 21); Vancouver Island.

S. riparius spreng.

Generally distributed, Vancouver Island-Alberni; Victoria.

S. robustus Pursh. (Salt-marsh Bulrush.)

Generally distributed, Vancouver Island.

S. subterminalis Torr.

Iare. Strathcona Park, T.I., J. M. Mucoun; Iount Mark.

S. validus Vahl. (American Great Bulrush.)

Generally distributed, Vancourer Islaud.

\section{ARACEÆ (Arum Family).}

Lrsichitor (Yellow Arum).

L. kamtschatcense (L.) Schott. (Slinnk Cabbage.)

Common. Vancourer Islaud.

\section{LEMNACE $巴$ (Duckweed Family).}

Lesisa (Duckweed).

L. minor L. (Lesser Duckweed.)

Generally distributed, Vancouver Islaud, Iacoun.

L. trisulca L. (Irs-leaved Duckweed.)

Locally distributed, Vaucouver Island-Alberni : Nanaimo: Cedar Hill.

SPIROdela.

S. polsrhiza (L.) Schleid. (Greater Duckweed.)

Locally distributed, Tancouver Island-Bearer Lake, Iucoun.

JUNCACE E (Rush Family).

Jux̃Cus (Rush).

J. acuminatus Michx. (Sharp-fruited Rush.)

Locally distributed, Shawnigan Lake, V.I., Wm. M. Canby. 
Juxcus-Continued.

J. alpinus var. fuscescens Fernald.

Locally distributed, Vancourer Island-Cameron Lake; Strathcona Park, J. II. Macoun.

J. alpinus rar. insignis Fries.

Common. Southem half of Vancourer Island, Macoun.

J. articulatus $\mathrm{L}$.

Locally distributed, Shawnigan Lake, V.I., Wm. MI. Canby.

J. balticus Willd. (Baltic Rush.)

Common. Vancourer Island; Queen Charlotte Islands.

J. Bolanderi Engelm.

Generally distributed, Vancourer Island.

J. brericaudatus (Engelm.) Fernald.

Ucluelet, V.I.

J. bufonius L. (Toad Rush.)

Generally distributed, Vancourer Island.

rar. fasiculiflorus Boiss.

Generally distributed, Vancouver Island.

J. Regelii Buck.

Locally distributed, Vancouver Island-Tictoria Al'm; Shawnigan; Bare lsland.

J. Covillei L'Per.

Vancouver Island-Comox; Nanaimo; Alberni.

J. effusus L. (Common Rush.)

Common. Tancourer Island.

rar. compactus L. \& C.

Lcluelet, V.I.

var. gracilis Hook.

Common. Vancourer Island.

var. hesperius Piper.

Vaucouver Island-Lcluelet; Departure Bay; Sidney, Macoun.

rar. pacificus Fernald \& Wiegand.

Generally distributed, Vancouver Island.

J. ensifolius Wiks.

Common. Vancouver Island; Queen Charlotte Islands.

var. major Hook.

Generally distributed, Vancouver lsland.

J. falcatus Meyer.

Vancouver Island-Alberni; Comox; Cowichan; Shamnigan. Mayne Island. var. alascensis Coville.

Locally distributed, Vanconver Island-Renfrew District, Rosendahl; Kennedy Lake; Strathcona Park.

J. Gerardi Loisel. (Black Grass.)

Vancouver lsland—Comox; Nanaimo.

J. Lescurii Boland.

Generally distributed, Vancouver Island.

J. Merteisianus Bong. (Alpine Bog Rush.)

Generally distributed in mountain marshes, Vancouver Island-Uclnelet;

Strathcona Park; Mount Arrowsmith.

J. Nevadensis Wats.

Locally distributed in marsles, Barkley Sound. Y.I.

J. nodosus L. (Kinotted Rush.)

Generally distributed, Vancourer Jsland.

J. occidentalis Wiegand.

Locally distributed, Vanconver Island-Sidney; Lllk Lake. 
Jexces-Coutinucd.

J. oreganus S. Wats.

Locally distributed, Vancourel Island-Cameron Lake; Shawnigan Lake.

J. orthophyllus Coville.

Locally distributed, Vancouver Island-Koksilah River.

J. oxymeris Engelm.

Locally distributed, Vancouver Island-Elk Lake.

J. Parryi Engeln.

Locally distributed in mountains, Vancourel Island-Ifount Benson;

Mount Millk; Mount Arrowsmith, Wacoun.

J. subtriflorus Coville.

Locally distributed in mountains, Vancourer Island-Mount Arrowsmith.

J. supiniformis Engelm.

Rarc. Alberui, V.I., Macoun.

J. tenuis Willd. (Slender Rush.)

Locally distributed, Vancouver Island-Elk Lake, Mucoun.

Lezela (Tood Rusli).

L. campestris (L.) DC. (Common Wood Rush.)

Common. Vancouver Island.

I. comosa E. Meyer.

Rare. Victoria, V.I., Macoun; Renfrew District, Roscndahl \& Brand.

L. divaricata S. Watson.

Vancourer Island. (Henry"s "Flora of Southern British Columbia.")

L. glabrata Desv.

Locally distributed, Mount Alrowsmith. Y.I., Ir. R. Carter.

L. parriflora (Ehrh.) Desv. (Small-flowered Mood Rush.)

Generally distributed, Vancouver Island; Mayne Island; Sisk, Skincuttle,

Skidegate Channel, Q.C.I. (Osgood. "North American Fauna," No. 21.)

L. spicata (L.) DC. (Spiked Wood Iiush.)

Locally distributed, Mount Alrowsmith; Mount Benson, V.I., Macoun.

L. subsessilis Wats.

Nootka, V.I.

\section{LILIACE $Æ$ (Lily Family).}

Asparagus.

A. officinalis. (Garden Asparagus.)

Escape from cultivation. Cowichan Bay, V.I.

Allucar (Onion).

A. acuminatum Hook.

Locally distributed, southern half of Vancouver Island.

A. cernuum Roth. (Nodding Wild Onion.)

Common. Vancouver Island.

A. cernuum Roth. forma alba.

Locally distributed, Cameron Lake, V.I., W. R. Curter.

A. crenulatum Wiegand. (Mountain Onion.)

Locally distributed, Mount Arowsmith, Y.I., Wacoun; mountains north of

Cowichan Lake.

A. Gejeri s. Wats.

Locally distributed. southern half of Vancouver Island-Victoria; Oak

Bay; Cadboro Lay; Barkley Sound; SIroat Lake; Sidney ; Maple Bay;

Cowichan. 
Camassia (Camass; Wild Hyacinth).

C. quamash (Pursh.) Greene.

Common. Southern half of Vaucouver Island.

C. Leichtliuii S. Watson.

Locally distributed, southern half of Vancourer Island.

C. Suksdorfii (Greenman) Piper.

Not common. Sidney, V.I., J. M. Macoun.

Clintonia.

C. uniflora (Schuit.) Kuntlı. (Queen Cup.)

Locally distributed. central portion of Vancouver Island-Alberni; Cowichats.

Disporuar (Fairy Bells).

D. oregallum (S. Wats.) B. \& H.

Conmon. Vancouver Island.

D. Smithii (IIook.) Piper.

Locally distributed, Vancouver Island-Nitinat River.

Erytironium (Adder's-tongue; Easter Lily).

E. giganteum Lindl. (White Easter Lily.)

Common. Vincouver Island.

E. Howellii Wats.

Rare. Cowichan Lake, J. R. Anderson; Barkley Sound, V.I., Hillier.

E. montanum Wats.

Rare. Great C'entral Lake, V.I., Frascr.

E. parviflorum (S. Wats.) Gooding. (Small Yellow Easter Lils.)

Rale. Mount Prevost, V.I.

E. Smitlii IIook. (Pink Adder's-tongue.)

Locally distributed, Vancouver Island-Nootka; Holberg; Alberui ; Cowichan; Nitinat.

Fritillaria (Fritillaria; Snake Lily).

F. camtschatcensis (L.) Ker-Gawl.

Locally distributed, Queen Charlotte Islands (Osgood, "North American

Fauna, No. 21). Tancourer Island-Alberni; Comox; Cowichan.

F. Janceolata Pursh.

Generally distributed, Vancouver Island.

Hookera (Wild IIyacinth).

H. coronaria Salisb.

Locally distributed, southern half of Vancouver Island-Albelni; Northfield; Cowichan ; Victoria.

II. Douglasii S. Wats. (WVild Hyacintlı.)

Locally distributed, near Victoria, V.I.; Quamichan.

H. hyacinthina (Lindl.) Kuntz. (Fool's Onion.)

Generally distributed, southern half of Vaneouver Island.

Liliua (Lily).

I. parviflorum (Hook.) Holtz. (Wild Tiger Lily.)

Common. Vancouver Island.

LLOTDIA.

L. serotina (L.) Sweet. (Mountain Spiderwort.)

Iiare. Strathcona Pirk, Y.I., J. M. Mucoun. 
MaIaxthencil (Trild Lily-of-the-Talley).

II. bifolium (L.) rar. kantschaticum Gmel.

Conmon. Queen Charlotte Islands; Vaneouver Island.

Nothoscondey (Yellow False Garlie).

r. bivalie (L.) Britton.

Plant collected by Jack Miller on Foul Bay Roar, Victor'ia, Y.I.

SMllacixa (False Solomon's Seal).

S. racenosa (I.) Desf. (False Spikenard.)

Generally distributcd, Vancouver Island. (S. brachypetala Rydb. and S. amplexicaulis Nutt. are referred to muter this species.)

S. sessilifolia Nutt.

Generally distributed, Vancourer Island.

S. stellata (L.) Desf. (Star-flowered Solomon's Seal.)

Common. Queen Charlotte Islands; Vancourer Island.

Stexaxthium.

$\therefore$. occidentale Gray. (Westeln Stenanthium: Bronze Bells.)

Locally distributed, Vancouver Island-Holberg; Beaufort Range, Alberni ; Iount Arrowsmith Trail.

STREPTOPL's (Twisted-stalk).

S. amplexifolius (L.) DC. (Clasping-leared Twisted-stalk.) Common. Queen Charlotte Islands; Vancouver Island.

S. roseus Michx. (Sessile-leared Twisted-stalk.)

Localls distributed, Queen Charlotte Islands (Osgood, "North American Fauna," No. 21.) Vancourer Island-Nitinat; Cowichan.

Tofaldia (False Asphodel).

T. intermedia Rydb.

Generally distributed, Queen Charlotte Islands; Vancourer Island.

T. occidentalis.

Yakoun Lake, Q.C.I.

Trulum (Wake Robin).

T. oratum Pursh.

Common. Vancourer Island.

Veratrum (False Hellebore).

V. viride Ait.

Generally distributed, Queen Charlotte Islands; Vanconver Island.

ZrGadexus (Zrgadene).

Z. venenosus Wats. (Poison Camass.)

Generally distributed, southerm half of Vancouver Island.

IRIDACE E (Iris Family).

HYDAsTrLes (Tellow Sisyrinchium).

H. borealis Bickn.

Locally distributed, Fancouver Island-Alberni; Caje Scott; Cameron

Lake; Shawnigan Lake; Cowichan.

Inis (Fleur-de-Lis; Flag).

I. versicolor L. (Large Blue Flag.)

Ecluelet, Y.I.. Масони. 
Sistrinchidu (Blue-ejed Grass).

S. birameum Piper.

Generally distributed, Vancouver Island.

S. grandiflorum Dougl,

Locally distributed, southeru end of Vancouver Island-Victoria.

S. idahoënse Beckn.

Generally distributed, Vancourer Island.

S. littorale Greene.

Locally distributed, Queen Charlotte Islands (Osgood, "North American

Fauna," No. 21). Vancouver Island-Nootka; Ucluelet; Victorla.

S. Macounii Bickn.

Locally distributed, Yancourer Island-Comox, Macoun; Ucluelet.

S. segetum Bickn.

Locally distributed, Sproat Lake Falls, V.I.; Cameron Lake, V.I., W. R. carter.

\section{ORCHIDACE E (Orchid Family).}

Calypso (False Lady's Slipper).

C. bulbosa (L.) Oakes.

Generally distributed, Queen Charlotte Islands; Vanconver Islaud.

Crpripedium (Lady's Slipper; Moccasin Flower).

C. montanum Dougl.

Rare. Apparently extinct on Vancouver Island; originally found near Victoria.

Corallorrhiza (Coral Root).

C. innata R. Br. (Early Coral Root.)

Mount Arrowsmith, V.I., Macoun.

C. maculata Raf. (Spotted Coral Root.)

Common. Vancouver Island.

C. Mertensiana Bong. (Spurred Coral Root.)

Locally distributed, Vancouver Island-Beaufort Range; Cowichan Lake; Queen Charlotte Islands, Dawson.

C. striata Lindl. (Striped Coral Root.)

Generally distributed, Vancouver Island.

LPIPActis (Rattlesnake Plantain).

E. decipiens (Hook.) Ames. (Menzie's Rattlesuake Plantain.)

Common. Queen Charlotte Islands; Vancouver Island.

Habenaria (Rein Orchid).

II. bracteata (Willd.) R. Br. (Long-bracted Orchis.)

Locally distributed, Goldstream, V.I.

H. dilatata (Pursh.) Gray. (Tall White Bog Orchis.)

Generilly distributed, Queen Charlotte Islands; Vancouver IsIand.

II. elegans Lindl.

Generally distributed, Vancouver Island.

II. graminifolia liydb.

Locally distributed, Cameron Lake, V.I.

II. leucostachys (Iindl.) Wats. (Giant Orchis.)

Common. Queen Charlotte Islands; Vancouver Island.

H. Michreli Greene.

locally distributed, Victoria, V.I. 
IIABEXARIA-Continucd.

II. orbiculata (Pursh.) 'Torr. (Large Round-leaved Orchis.)

Rare. Strathcona Park. V.I.; W. B. Anderson.

II. stricta Lindley. (Green Bog Orchis.)

Locally distributed, Tancouver Island-Beaufort Range; Mount Arrowsmith; Cowichan Lake. Queen Charlotte Islands.

II. unalaschensis (Spreng.) Wats. (Alaska Orchis.)

Generally distributed, Queen Charlotte Islands; Vanconver Island.

H. viridiflora (Cham.) Rydb. (Tall Leafy Green Orchis.)

Locally distributed, Iount Brenton, Y.I.

Listera (Twayblade).

L. caurina Piper. (Testern-wood Twayblade.)

Generally distributed, Vancouver Island.

L. convallarioides (Sw.) Torr. (Broad-lipped Twayblade.)

Locally distributed, Queen Charlotte Islands-Tasu Harbour, $D r, C . F$.

Tencombe. Vancourer Island-Alberni; Mount Prevost.

L. cordata (L.) R. Br. (Ieart-leaved Twayblade.)

Generally distributed, Queen Charlotte Islands; Vancourer Island.

Spiraxtmes (Ladies Tresses).

S. Romanzoffiana Cham.

Generally distributed, Queen Charlote Islands; Tancouver Island.

Salix (Willow).

\section{SALICACE正 (Willow Family).}

S. Geyeriana Anders.

Locally distributed, Vanconver Island-Saanich, Macoun.

S. Hookeriana Barr.

Common. Vancouver Island.

var. laurifolia Henry.

Tocally distributed, Alberni, V.I.

S. lasiandra Benth. (Black Willow.)

Common. Vancouver Island.

S. Mackenziana (Hook.) Barr.

Not common. Vancouver Island-Victoria.

S. macrostachya Nutt.

Locally distributed, Koksilah River, V.I.

S. melanopsis Nutt.

Locally distributed, Cowichan River, T.I.

S. prolixa Auders.

Victoria, Y.I., Macoun.

S. sitchensis (Sanson) Bong. (Sitka Wrillow.)

Common. Queen Charlotte Islands; Vancouver Island.

S. Scouleriana (Hook.) Barr.

Common. Queen Charlotte Islands; Vancouver Island.

Populus (Poplar).

P. tremuloides Michx. (Aspen.)

Locally distributed, Vancourer Island-Alberni; Cowichan; Victoria.

P. trichocarpa T. \& G. (Black Cottonwood.)

Generally distributed, Vancouver Island.

P. Tancouveriana Trelease.

Locally distributed, Sidney, V.I., Macoun. 


\section{MYRICACE王 (Bay-berry Family).}

Mrrica (Bay-berry).

M. Gale L. (Sweet Gale.)

Common. Queen Charlotte Islands: Vancourer Island.

\section{BETULACE正 (Birch Family).}

Alnus (Alder).

A. rubra Bong. (Red Alder.)

Common. Queen Charlotte Islands; Vancourer Island.

A. sitchensis (Regel.) Sarg. (Green Alder.)

Genelally distributed in higher elevations on Vancourer Island; Queen

Charlotte Islands, Dr. C. F. Neucombc.

Betula (Birch).

R. Hallii Howell.

Locally distributed, Lost Lake, V.I., C. C. Pcmberton; Macoun.

B. glandulosa Michx. (Dwarf Birch.)

Locally distributed, Mount Douglas ("Cedar Hill"), J. R. Anderson.

B. occidentalis IInok. (Western Birch.)

Locally distributed, near Victoria, V.I.

Coryus (Hazel).

C. rostrata Ait. var. californica A. DC.

Locally distributed, Goldstream, V.I., Ifacoun.

\section{FAGACEE (Beech Family).}

Quercis (Oak).

Q. Garlyana Dongl. (Garry Oak.)

Locally distributed. Victoria, and southern end of Vanconver Island.

HuMulus (Hop).

\section{URTICACE正 (Nettle Family).}

H. Lupulus L. (Common Hop.)

Generally distributed, Vancouver Island. (Escaped from cultivation.)

CuMUs (EIm). (English Elm.)

$\tau$. campestris $L$.

Locally distributed, Tancouver Island-Saanichton; Sidney.

Lrtica (Nettle).

L. Lyallii Wats. (Stinging Nettle.)

Common. Tancouver Island; Queen Charlotte Islands.

U. urens. L. (Small Nettle.)

Locally distributed, Victoria, V.T.

\section{SANTALACEÆE (Sandalwood Family).}

l'omanlli (Tastalel Toad-flax).

C. Iilchardsiana Fernald.

Locally distributed, Laumford Lake, V.I., Macoun.

LORANTHACEE (Mistletoe Family).

ARCEUTHOBUM (Mistletoe).

A. americanmm Nutt.

Locally distributed, Vietoria. V.I., Iracoun. 
ArCelthobila-comtinucl.

A. tsugensis Rosendaht.

Locally distributed, Vancouver Island, west coast.

\section{ARISTOLOCHIACEE (Birthwort Family),}

Asarem (Wilel Ginger).

A. caudatum Lindl.

Vancourer Island-Comox: Alberni; Sahtlam.

OXYRIA.

\section{POLYGONACE无 (Buckwheat Family).}

O. digrna (L.) Hill. (Mountain Sorrel.)

Rare. Mount Arrowsmith, V.I., TV. R. rarter.

Polygoxu (Knotreed).

P. amphibium L. (Willow Weed.)

Generally distributed, Vancourer Island.

P. amphibium var. Hartwrightii (Gray) Bissell.

Locally distributed. Vancouver Island-Colquitz River.

I'. ariculare L. (Door Ireed.)

Common. Tancourer Island.

var. regetum Ledeb. (Ḱnot Grass.)

Generally distributed, Vaucourer Island.

P. buxiforme small.

Locally distributed, Victoria, V.I., Macoun.

P. Convolvulus L. (Bindweed.)

Common. Vancourel Island.

I'. Douglasii Greene. (Douglas' Knotweed.)

Locally distribnted, southern half of Vancouver Island-Comox; Alberni;

Cameron Lake.

P. emersum (Michx.) Britt.

Locally distributed, Tancourer Island-Comox.

I'. Fowleri Robinson. (Fowler's Knotweed.)

Locally distributed, Vancouver Island-Tictoria.

P. Hydropiper L. (Smartweed.)

Common. Tancouver Island.

P. hydropiperoides Michx. (Wild Water-pepper.)

Vancourer Island-Beaver Lake.

I'. lapathifolium L. (Pale Persicaria.)

Locally distributed, Tasu Harbour, Queen Charlotte Islands. Vancouver Island-Comox; Cowichan River; Sidney.

P. minimum S. Wats.

Rare. Mount Benson, V.I., Macoun.

P. Nuttallii Small.

Rare. Mount Mark, V.I., Mucoun; Cowichan Lake, V.I.

P. paronychia C. \& S.

Locally distributed, southern half of Vancouver Island-Comox; Victoria.

P. Persicaria L. (Lady's Thumb.)

Generally distributed, Vancourer Island.

P. rubescens Small.

Locally distributed, Victoria, V.I., Macoun.

P. sachalinense S. Schmitt.

Locally distributed, Táncouver Island-Siduey; Mayne Island.

P. sjergulariaforme Meisn.

Common. Vancouver Island. 
Porrgonua-Continucl.

P. tomentosum Schrauk.

Locally distributed, Vanconver Island, near Victoria.

Rustex (Dock).

R. Acetosa L. (Sour Dock.)

Not common. Vancouver Island-Victoria.

R. Acctosclla L. (Sheep Sorrel.)

Common. Tancourer Island.

R. conglomeratus Murr. (Clustered Dock.)

Common. Vancourer Islaud.

R. crispus L. (Curled Dock; Yellow Dock.)

Generally distributed, Vancourer Islaud.

R. hesperius Greene.

Locally distributed, Vancouver Island-Deep Cove, Macoun.

R. obtusifolius L. (Broad-leaved Dock.)

Generally distributed. Vancouver Island.

R. occidentalis S. Wats. (Westerm Dock.)

Common. Vaucourer Islaud.

R. persicarioides L. (Golden Dock.)

Generally distributed. Vancouver Island.

R. pulcher L. (Fiddle Dock.)

Locally distributed, Mayne Island, Macoun.

R. mexicanus Meisn.

Common. Queen Clarlotte Islands. Vancourer Island-Nanaimo; Lcluelet; Cowichan Lake.

\section{CHENOPODIACEE (Goosefoot Family).}

Atriplex (Orach).

A. Gmelini C. A. Meyer.

Locally distributed, Queen Charlotte Islands. (Osgood, "North American Fauna," No. 21.)

1. hastata L,

Locally distributed, Skidegate, Queen Charlotte Islands. Vanconver Island-Comox; Victoria.

A. patula I. var. littoralis Gray.

Generally distributed, Vancouver Island.

A. zoster'solia (Hook.) Wats.

Locally distributed, Knnox Harbonr, Dawson.

Chenoponium (Goosefoot).

C. album I. (Lamb's Quarters; Pigweed.)

Common. Vancouver Island.

C. Iybridum I. (Maple-leaved Goosefoot.)

Generally distributed, Vancouver Island.

(. murale I. (Nettle-leared Goosefoot.)

Generally distributed, Vancouver Island.

C. urbicum L. (City Goosefoot.)

Iocilly distributed, Vinconver Island-Victoria; Nanaimo.

Saliconxia (Glasswort).

S. ambigua Nichx.

Generally distributed, Queen Cluarlotte Islands. Tidal Flats, Vancourer lsland. 


\section{SALICORXIA-Contilluce.}

S. herbacea $\mathbf{L}$.

Locally distributed, Queen Charlotte Islands. (Osgood, "North American Fama," No. 21.)

SALsol. (Saltwolt; Russian Thistle).

S. Kinli L. rar. tenuifolia G. F. W. Mer. (Saltwort.)

Locally distributed, Nanaimo, Y.I.. Macoun.

ṠUAEDA (Sea 13lite).

s. maritima (I.) Dumont.

Locally distributed, Vancourer Island-C'omox. Macoun.

\section{AMARANTHACEE (Amaranth Family).}

AMaraxtiles (Amalanth).

1. blitoides Wats. (Prostrate Amarantlu.)

Generally distributed, Tancouver Island-Departure Bar.

A. getaceisaus 1. (Tmmble Weed.)

Locally distributed, Vancouver Islaur-Departure Bay.

1. retroflexus $\mathrm{I}$.

Victoria, V.I., Fletcher.

Aeroxia.

\section{NYCTAGINACE王 (Four-o'clock Family).}

A. latifolia Esch. (Yellow Sand Terbena.)

Common. Sindy beaches, Masset, Q.C.I. Vancouver Island-Victuria;

Long Beach.

A. umbellata Lam. (Pink Sand Verbena.)

Locally distributed, Pachena Bar, T.I., Jas. Fraser; Ahouset, V.I., Dr. C. F.

Nexeombe.

\section{CARYOPHYLLACE正 (Pink Family).}

Arevaria (Sandwort).

A. capillaris Poir. var. nardifolia Rydb. (Hair-leaved Sandwort.)

Locally distributed, Carmanah, V.I., J. R. Anderson.

A. lateriflora L. (BIunt-leaved Sandwort.)

Rare. Queen Charlotte Fslands, Dr. C. F. Newcombe; Alberni, T.I.

A. macrophylla Hook. (Large-leaved Sandwort.)

Common. Vancouver Island.

A. peploides L. (Sea-beach Sandwort.)

Commou. Vancouver Island.

A. peploides rar. oblongifolia Trats.

Locally distributed, coast of Queen Charlotte Islands. Vancourer Island-

Qualicum; Long Beach; Victoria.

A. serp!llifolia I. (Thyme-leared sandwort.)

Victoria, Y.I., Fletcher.

A. tenella Nutt. (Slender Sandwort.)

Locally distributed, sonthern end of Vancouver Island, near Victoria.

A. verna L. (Yernal Sandwort.)

Locally distributed in mountains, Vancourer Island.

var. propinqua (Rich.) Fernald.

Locally distributed. Vancouver Island-Mount Arowsmith. 
Agrostemia.

A. Githago L. (Corn Cockle.)

Locally distributed, Cowichan River. V.I. (Glendemning's List, 1918.)

Cerastium (Mouse-ear Chickrweed).

C. alpinum var. Fischerianum (Alpine Chickweed.)

Locally distributed, Queen Charlotte Islands, Dr. C. F. Neweombe.

C. arrense L. (Field Chickweed.)

Common. Tancourer Island.

c. campestre Greene.

Locally distributed, Vancouver Island-Tidal Flats, Parksville.

C. graminifolium (Greene Rydb).

Generally distributed, Vancourer Island.

c. glomerutum Thuill.

Locally distributed, Vancouver Island-Victoria.

C. nutans Raf. (Nodding Chickweed.)

Locally distributed, southern end of Vancourer Island-Kyuquot; Victoria.

C. semidecandrum L.

Locally distributed, Yancouver Island-Cadboro Bay; Yictoria.

C. strictum L.

Locally distributed, Queen Charlotte Islands, Dr. C. F. Neueombe.

C. riscosum L. (Mouse-ear C'hickweed.)

Common. Vancouver Island.

C. vulgatum L. (Larger Mouse-eared Chickweed.)

Generally distributed, Tancouver Island.

DinNthus (Pink).

D. Armeria L. (Deptford I'ink.)

Locally distributed, Victoria, Y.I.

D. deltoides L. (Meadow Pink.)

Locally distributed, Victoria. V.I.

LYCHNIS.

L. eoronaria (L.) Desv. (Mullein Pink.)

Locally distributed, Nanaimo, V.I.

L. vespcrtince sibtl. (White Campion.)

Locally distributed, Mayne Island, IIacoun.

Sigiva (learlwort).

s. crassicalulis Wats. (Fleshy Pearlwort.)

(Gencrally distributed, Queen Charlotte Islands; Tancourer Island.

S. occidentalis (ireene. (Testern Pearlwort.)

Generally distributed, Queeu Charlotte Islands; Vaucouver Island.

S. stricta Fries.

Locally distributed, Vancouver Island-Langford Lake.

SAPONARIA (SOapTOI't).

S. officinalis L. (Bouncing Ret.)

Locally distributed, Vancouvel Island-Sidney.

s. Vaccaria L. (Cowherb.)

Locally distributed, Vancouver Island-Victoria; Alberni; Cowichan.

Sipergula (Slurity).

S. arrensis L. (I'overty Weed.)

Common. Vancouver Island.

s. satila lioenus.

fiencrally distributed. Vancoured Island. 
Spergulatia (Simd Sphry

S. macrothecal Hornem.

Locally distributed. Vancourer Island-Victorial; Barkley Sound, Macoun.

S. marina (I.) Griseb. (Salt-marsh Sand Spurry.)

Locally distributed, Queen Charlotte Islands (Osgood. "Fortlı American

Fauna," No, 21). Vanconver Island-Comox; Victoria.

S. rubra (L.) J. \& C. Presl. (Sand Spurry.)

Common. Vanconver Island.

S. salinal var. leiosperma Kindb.

Loeally distributed, Vancouver Island-Sidney.

Sillexe (Catchfḷ; Campion; Wild Pink).

S. acaulis L. (Moss Campion.)

Rare. Mount Arrowsmith, T.I., Fermode and Carter.

S. antirrhina Linn. (Sleepy Catchfly.)

Locally distributed, Vancouver Island-Malahat; Troulalem.

S. Armeria L. (Sweet William Catchfly.)

Locally distributed, Victoria, T.I.

S. Douglasii Hook.

Locally distributed, Momnt Benson, V.I.

S. gallica L.

Locally distributed, sontlern half of Vanconver Islaud-Departure Bay;

Cowichan; Victoria.

S. latifolia (Mill.) Brit. \& Ren.

Cowichan, T.I. (Glendemning's List, 191S.)

S. Menziesii Hook. (Menzie's Pink.)

Generally distributed, Vancourer Island.

$\therefore$ noetiflora L. (Night-flowering Catchfly.)

Locally distributed, Vancouver Island-Comox; Victoria.

S. Scouleri Hook. (Scouler's Pink.)

Locally distributed, sonthern end of Tancouver Island-rictorial.

Stellaria (Chickweed).

S. aruatica (L.) Scop. (Water Chickweed.)

Locally distributed, Nanaimo, T.I.

S. borealis Bigel. (Northern Stitchwort.)

Queen Charlotte Islands. Tancourer Island-Beaver Lake: Aberni;

Thetis Lake.

var. alpestris Brit.

Vancourer Island-Duncan.

var. Bongardiana Fernald.

Tancourer Island-Sidney.

var. sitchana (Steud.) Feruald.

Vancourer Island-Sidney.

S. crispa Cham. \& Schlecht.

Tancourer Island-Victoria; Sidnes; Alberui; Clayoquot.

s. hrmmifusa Rottb.

Locally distributed, Vancourer Island-Ccluelet; Newcastle Island.

S. humifusa oblongifolia Fenzl. (Marsh Stitchwort.)

Blenkinsop Bay.

S. longifolia Muhl. (Long-leared Stitchmort.)

Generally distributed, Vancourer Island.

S. media (L.) Cyrill. (Common Chickweed.)

Common. Vancouver Island. 
STELLARIA-Continued.

S. nitens Nutt.

Very locally distributed, southern end of Vancouver Island, around Victoria, IIill and Macoun.

S. strictifiora Rydb.

Locally distributed, Vanconver Island-Beaver Lake.

S. stricta Richards var, maritima Fries.

Locally distributed, Langford Lake, V.I.

\section{PORTULACACEÆ (Purslane Family).}

CALANDRINIA.

C. caulescens Menziesii (Hook.) A. Gray.

Locally distributed, southern end of Vancouver Island-Tzouhalem; Victoria.

LEWISIA.

L. columbiana (Howell) Rob.

Locally distributed, IIomnt Arrowsmith and mountains north of Cowichan

Lake, V.I.

L. pjgmaa (Gray) Rob.

Rare. Mount Arrowsmith, V.I., Kermode and Carter.

Montia (Blinks).

M. asarifolia (Bong.) Howell.

Common. Masset, Queen Charlotte Islands; Vancouver Island. (Henry"s

"Flora of Southern Irritish Columbia.")

M. Chamissoi (Ledeb.) Dur, \& Jackson.

Locally distributed, Comox, Y.I. ; Alberni. V.I.

II. dichotoma (Nutt.) Howell.

Locally distributed. southern half of Vancourel Island-Victoria; Wellington.

II. diffusa (Nutt.) Greene.

Locally distributed, Vancouver Island-Cameron Lake, J. R. Anderson; Alberni, J. K. Henry.

II. fontana L. (Blinking Chickweed.)

Common, Queen Charlotte Islands; Tancouver Island.

II. Howellii Wats.

Locally distributed, Vancouver Island-Victoria ; Triangle Island, Dr: C. $F$. Tercombe.

II. linearis (Dougl.) IIowell.

Common. Vancouver Island.

II. parviflora Dougl.

Common. Vancouver Island.

Ir. parviflora rar. depressa (Rob.) Gray.

Locally distributed, Vancouver Island--Alberni. Roberts Bay.

II. parrifolia IIoc. (Small-leaved Spring Beauty.)

Common. Queen Charlotte Islands; Vancouver Island.

I. perfoliata (Domn.) Howell. (Spanish Lettuce.)

Commou. Vancouver Island.

M. sibirica (I.) IJowell. (Spring-beauty; Miner's Lettnce.)

Common. Vanconver Island; Queen Charlotte Islands.

I. spathulata (Dougl.) Iowell.

Generally distriluted, southern half of Tancouver Island.

Portulaca.

$P$. aleraced L. (Purslane).

Locally distributed, cowichan, V.I. 


\section{CERATOPHYLLACERE (Hornwort Family).}

Ceratopitilet.

C. demersum 1 .

Rare. comox, V.I., Mecomul.

\section{NYMPHAECE无 (Water Lily Family).}

BRASENIA.

F. Schreberi Gmel. (Watel Shield.)

Locally distributed. Vaucomer Island-Loon Iake, Alberni ; Fourth Lalie, near Victoria. Texada Island.

YYurir.eA (Water Lily).

․ polysepala Englem. (Yellow Pond Lily.)

Common. Queen Charlotte Islands; Tancourer Island.

\section{RANUNCULACEE (Crowfoot or Buttercup Family).}

Acovitum (Monkshood).

A. delphinifolium DC. (Wild Monkshood.)

Rare. Queen Charlotte Islands, C. deB. Green.

Act.en (Baneberry).

A. arguta Nutt. (Red Baneberry.)

Generally distributed, Vancourer Island.

A. eburnea Rydb. (Thite Baneberry.)

Rare. Tancouver Island-Sproat Lake; Henderson Lake.

ANEMONE

A. Lyallii Britt.

Vancouver Island-Wootka; Comox; Alberni; Qualicum; Mount Benson;

Henderson Lake: Cowichan; Nitinat; Malahat: Sidney; Esquimalt.

1. multifida Poir. (Trind Flower.)

Rare. Koksilah, V.I., Worsfold. Limestone Islands, Q.C.I., Dr. C. F. Tercombe.

A. narcissiflora $\mathrm{I}$.

Locally distributed, Qneen Charlotte Islands. (Osgood, "North Ameriman Fauna," No. 21.)

1. occidentalis Freyu. (Pasque Flower.)

Hare. Stratbeona Park, V.I., J. M. Macomm.

Aqurlegia (Columbine).

A. formosa Fischer. (Wild Columbine.)

Common. Queen Charlotte Islands; Vanconver Island.

4. vulgaris L. (European Columbine.)

Locally distributed, Siclnes. V.I., Macoun.

CALTHA.

C. asarifolia DC.

Rare. Comox, V.I., J. R. Anderson; Gawi, Q.C.I., Dr: C. F. Nencombc.

C. biflora DC.

Queen Charlotte Islands, Dr. C. F. Tevcombc.

C. leptosepala DC. (Alpine Marigold. )

Common in mountain marshes, Queen Charlotte Islands: Vancouver Island.

C. palnstris L. (Marsh Marigold.)

Locally distributed, Tancouver Island-Comox: Mount Arrowsmith, Macoun. Queen Charlotte Islands. (Osgood, "North American Fauna," No. 21.) 


\section{Clematis.}

\section{C. ligusticifolia Nutt.}

Rare. Running wild at Departure Bay, V.I.

\section{Coptis (Gold Thread).}

C. asplenifolia Salisb.

Generally distributed, Queen Charlotte Islands. Vancouver Island-Cum-

berland; Francis Lake; Celuelet.

C. trifolia (L.) Salisb.

Rare. Mount Mark, Y.I., Macoun.

Delphiniua (Larkspur).

D. Ajucis I. (Rocket Larksiur).

Gallden escape, Vancourer Island-Victoria: Sidney.

D. Menziesii DC. (Menzie's Larkspur.)

Common. Tanconver Island.

\section{Raxunculus (Buttelemp).}

\section{R. acris L. (Tall Meadow Buttercup.)}

Locally distributed, Vancourer Island-Comrtenay ; Comox.

I. alismaefolius Geyer.

Locally distributed, near Victoria, V.I., Iucoun.

R. aquatilis I. (Water Buttercup or Crowfoot.)

Common. Vinconver Island.

var. capillaceus DC.

Generally distributed, Tancouver Island.

Ii. Bongardi Greene. (Small-flowered Buttereup.)

Common. Vanconver Island.

val. Douglasii Howell.

Common. Vancouver Islaud.

R. circinatus Sibth.

Rare. Comox, V.I., J. R. Anderson.

R. (ymbalaria I'ursh. (Seaside Crowfoot or Buttercup.)

Locally distributed. Vancouver Island-Parksville.

var. saximontanus Fermald.

Locally distributed, Vancourer Island-Siduey.

Ii. Exchscholtzii Schlecht. (Alpine Buttercup.)

Common in mountains, Vancouver Island.

I. Flammula var, reptans (L.) Mey, (Creeping Spearwort.)

Common. Vancouver Island.

var. unalaschensis (Bess.) Ledeb.

Generally distributed, Vancourer Island.

R. hebecarpus Hook \& Alu.

Locally distributerl, Tancourer Island-Nanaimo, Macoun.

R. Lobbii A. Gray. (Watel Buttercup.)

Rare. Lost Lake, V.I.

Ii. Macounii Britton. (Macomns Buttercup.).

Iiare. Victria, V.I., , R. Auderson.

R. occidentalis Nutt. (Westeru Buttercup.)

Common. Queen Charlotte Islands; Vaucourer Island.

R. orthorhynchus 110ok. (Magnificent Iiuttercup.)

Vanronver Island-Comox; Aberni ; Wellington; Cowichan; Sidney; Victoria.

var. platyphyllus A. Gray.

Generally distributed, Vancouver Islanol. 
Raxuxculs-Continued.

R. pennsclranicus L. (Bristly Buttercup.)

Locally distributed, Vancourer Island-Celuelet.

R. repcus L. (Creeping Buttercup.)

Generally distributed, Vancourer Island.

R. sceleratus L. (Celery-leaved Buttercup); Cursed Buttercul).)

Iiare. Ibare Island, W. B. Aulcrsom.

Mrosures.

II. major Greene. (Monse-tail.)

Locally distributed, near Victoria, V.I., Hucoun.

Trialctrua (Meadow Rue).

T. occidentale 1. Gray. (Western Meadow Iine.)

Generally distributed, Queen Charlotte Islauds: Vancourer Island.

Trautvetteria.

T. grandis Nutt. (False Bugbane.)

Locally distributed, Vancouver Island-Hardy Iay; Comox; Alberui;

Cowichan: San Juan.

Trollies.

T. laxus Salish. (Globe Flower.)

Iare. Stratheona Park, Y.I.: Cowichan Lake. Y.I.

BERRERIS.

\section{BERBERIDACE正 (Barberry Family).}

I3. aquifolium Purslı. (IIolly-leared Iahonia.)

Common. Vancouver Island.

B. nerrosa Pursh, (Oregon Grape.)

Common. Vancourer Island.

B. repens Lindl.

Vaucourer Island (Fletcher). (Macoun's "Catalogue of Plants.")

ACIILYS.

A. triphylla (Smith) DC. (May Leaves.)

Tancourer Island-Alberni: Comox; Nanamo: Qualicum: Cowichan;

Shawnigan; Goldstream; Sidney: Esquimalt.

\section{Eschischoltzia.}

\section{PAPAVERACE $\mathbb{E}$ (Poppy Family).}

E. reeta.

Garden escape, Cameron Lake, V.I.

E. culifornica Cham.

Garden escape. Victoria, I.I.: Siduer, V.I.

P'Latístigara.

P. oresanum (Nutt.) I. \& H.

Locally distributed, Cedar Hill, V.I.; Mount Finlarson, V.I

\section{FUMARIACE无 (Fumewort Family).}

Corydalis (Fumitory).

C. Scouleri Hook. (Scouler's Corydalis.)

Rare. Nitinat River, Y.I., W. R. Carter.

C. aurea Willd. var, occidentalis Engelm. (Golden Coryitilis.)

Found on Vancourer Island. (Macoun's "Catalogue of Plants.") 
DICENTRA.

D. formosa (Andr.) DC. (Bleeding IIeart.)

Common. Tancouver Island.

\section{CRUCIFERE (Mustard Family).}

ARABIS (Rock C'ress).

A. Breweri Wats.

Locally distributed, Tzouhalem, V.I. ; Strathcona Park, V.I.

A. Drummondii Gray. (Drummond’s Rock Gress.)

Locally distributed morth of Cowichan Lake, V.I.

A. glabra (L.) Bernh. (Tower Mustard.)

Generally distributed, Vancouver Island.

A. hirsuta (L.) Scop. (Hairy Rock Cress.)

Generally distributed, Vancouver Island; Queen Charlotte Islands.

A. holbœllii Hornem. (Holboll's Rock Cress.)

Victoria, V.I., Macoun.

A. IIookeri Lange.

Iare. Cowichan Lake, V.I.

A. lyrata L. rar. occidentalis Wats. (Lyre-leaved Rock Cress.)

Locally distributed, Vancourer Island-Cowichan Lake.

Athysanus.

A. jusillus (Hook.) Greene.

Localls distributed, southern end of Vancouver Island-Mount Finlayson;

Obselvatory Hill.

Barbarea (Winter Cress).

B. americana Rydb.

Locally distributed, Cowichan River, V.I.

B. orthoceras dolichocarpa Fernald. (Bitter Cress.)

Generally distributed, Queen Charlotte Islands; Vancouver Island.

B. rulgaris R. Br. (Yellow Rocket.)

Generally distributed, Queen Charlotte Islands; Tancouver Island; Triangle Island.

Brassica (Mustard; Turnip).

B. alba Gray. (White Mustard.)

Common. Vancouver Island.

B. arcusis (L.) Ktze. (Charlock.)

Common. Vancouver Island.

B. campestiv L. (Turnip.)

Common. Tancouver Island.

B. nigra (L.) Koch. (Plack Mustard.)

Common. Vancouver Island.

B. Rapa L. (Turnip.)

Common. Escaped from cultivation, Sidney, V.I.

B. sinapistrum Boiss. (Tild Mustard.)

Jocally distributed, Victoria, V.I.

\section{Cakile.}

C. edentular (Bigel.) Mook. (Sea Rocket.)

Locally distributed, coast of Vancourel Island-Tictoria; Long Beach;

Ccluelet; Aliousat. 


\section{CAMELINA}

C. satiru (L.) Crantz. (False or Dutch Flax.)

Locally distributed, Vancouver Island-Victoria.

Capsella

C. Bursu-pastoris (L.) Medic. (Shepherd's Purse.)

Common. Tamcourer Island.

Cardamise (Bitter Cress).

C. angulata Hook.

Rare. Queen Charlotte Islands, Dr. C.F. Tcucombc; Lake San Juan, T.I., Rosculahl.

C. Breweri Watson.

Locally distributed, Vancouver Island-Chemainus, J. R. Anderson.

C. intermedia IInlm.

Locally distributed, Vancouver Island-Victoria; Nanaimo; Tellington; Toluelet.

C. kamtschatica (Regel) Schultz.

C. umbellata Greene (according to Piper).

Slidegate, Q.C.I.; Victoria, V.I.

C. occidentalis (S. Wats.) Howell.

Locally distributed, Vanconver Island-Kennedy Lake; Uchelet.

C. oligosperma Nutt.

Common. Queen Charlotte Islands; Vanconver Island.

C. pemsylranica Muhl, (Pemsylvania Bitter Cress.)

Generally distributed, Vancouver Island.

C. pratensis L. (Meadow Bitter Cress.)

Locally distributed, skidegate, Q.C.I., Dr. C. F. Teucombe.

var. occidentalis.

Nanaimo, V.I., Macoun.

COCIILEARIA.

C. officinalis Lim. (Spoonwort.)

linre. Queen Charlotte Islands; Triangle and Solander Islands, Dr. C. F.

Icucombe; Cchucklesit, V'.I.

Conoxopes (Wart Cress).

C. didymus (L.) Sm.

Generally distributed, Nanaimo, V.I.

DEXTARIA (TOOthWOIt).

I). macrocarpa Nutt.

Locally distributed. Koksilah, V.I.; Burnside Foad, Victoria, V.I.

1). tenella Pursh.

Generally distributed, Vancourer Island.

Draba (Whitlow Grass).

I) Intea Gilib.

Victoria, T.I. (Henry"s "Flor"a of Soutbern British Columbia.")

D. nemorosa I. var. hebecarpa Lindblad. (Wood Whitlow Grass.)

Locally distributed, southern end of Vancouver Island-Tictoria.

D. nivalis Lilj. (Yellow Aretic Whitlow Grass.)

Rare. Mount Arrowsmith, Y.I., W. R. carter.

D. verma Linn. (Vernal Whitlow Grass.)

Locally distributed, Vanconver Island-Cowichan; Victoria; Cadboro Bay. 
Hesperis ( Rocket).

II. matronalis L. (Dame's Violet.)

Garden escape, Victoria, V.I.; Sidney, V.I.

\section{Hutchinsia.}

I. procumbens Desr. (Prostrate Hutchiusia.)

Locally distributed, Vaucouver Island-Beacon Hill. Victoria.

Erysiandu.

E. cheiranthoides L. (Treacle Mustard.)

Locally distributed, Vanconver Island--Hilliers; Cameron Lake.

E. elatum Nutt. (Western Wall-flower.)

Locally distribnted in momtains, Vancouver Island-Mount Arrowsmith and mountains north of Cameron Lake.

L. orientale $\mathbf{R} . \mathbf{B r}$.

Locally distributed, Sidney, V.I., MLacoun.

E. torulosum Piper.

Mount Arrowsmith, V.I., IF. R. Carter.

This plant must be segregated from E. asperm (Nutt.) DC on account of its sreen lrerbage and very different torulose pods. Perhaps all the specinlens from high altitudes in the Cascade and Olympic Mountains are to be referred here, lut until the present, mature pods seem not to have been collected. (C. V. I'iper.)

\section{LePIdium (Peppergrass).}

L. apetulum Willd.

Locally distributed, Bare Island, W. B. Anderson.

L. densiflorum Schw. (Wrild Tongue I'eppermlass.)

Locally distributed, Vancouver Island-Namaimo.

L. Drabu L. (Hoiry Cress.)

Locally distributed, Vanconver Island-Victoria, J. R. Anderson; Alberni.

L. medium Greene.

Locally distributed, Vanconver Island-Victoria.

L. Menziesii DC.

Locally distributed, Tancourer Island-Albelni; Parksville; Victoria.

I. oxycalpum T. \& $\mathrm{r}$.

Locally distributed, Tancouver Island, Macoun. (Piper and Beattie's

"Flora of the Northwest Coast.")

L. sativum L. (Garden Peppergrass.)

Generally distributed, Vancouver Island.

L. strictum Irattan.

Locally distributed, Vaneouver Island-Cadboro Bay.

Lopularia (Sweet Alyssum).

\section{I. maritima (L.) Dest.}

Locally distributed, Vancourer Island-Tictorial.

\section{Neslia.}

N. panirulata (L.) Dest.

Locally distributed, Vancourer Island-Sidney: Namimo; Courtenay; Alberni.

Nesodraba.

X. megalocirpa Greene.

Karc. 'Iriangle Island; Queen Charlotte Islands, Dr. C. F. Neweombe. 


\section{Platysieringar.}

I'. scrilujgerum Hook.

Rare. Mount Finlayson, V.I., Macom; Observatory Hill, V.I., Cumingham and $C . F$. Texeombe.

RADICLLA (Thatereress).

R. Armoracia (L.) Robinson, (Horseradish.)

Common. Garden escape, Vancouver Island.

R. curvisiliqua Hook.

Generally distributed, Vancouver Island.

R. indica L.

Locally distributed, Vancouver Island-Nanaimo.

R. Nasturtium-aquaticum (T.) B. \& R. (Watereress.)

Locally distributed, Vancourer Island-Nanaimo; Wellington; Sidney.

R. Nuttallii Rydb.

Iocally distributed. Vanconver Island-Beaver Lakc; Iost Lake.

R. obtusa (Nutt.) Greene. (Bilunt-leaved Ycllow Cress.)

Locally distributed, Vancouver Island-Namaimo.

R. pacifica (Howell) Greene.

Locally distributed, Vancourer Island-Victoria.

R. palustris (L.) Moench. (Marsh Cress.)

Locally distributed, Vancourer Island-Victoria.

Raphaxus (Radish).

R. satirus L. (Garden Radish.)

Common. Garden escape.

Sisyarbiua (Hedge Mustard).

S. altissimum. I. (Tumble Mustard.)

Common. Vancouver Island.

S. officinale (L.) Scopr. var. lelocaryum DC. (Hedge Mustard.)

Generally distributed, Vancouver Island.

Subularia (Awiwort).

S. aquatica L. (Water Awlwort.)

Rare. Sproat Lake, Great Central Lake. Quamichan Lake, V.I.

Thlaspi.

T. arrense I. (French Weed: Stinkweed.)

Locally distributed, Vancouver Island-Cowichan; Victoria.

'TiYsanocarpus (Lace I'od).

T. curvipes Hook.

Locally distributed, near Victoria. V.I.-Momnt Finlayson.

DroserA.

\section{DROSERACE正 (Sundew Family).}

D. anglica Huds. (Oblong-leared sundew.)

Locally distributed, Iorne Lake; Alberni, V.I.

D. rotundifolia L. (Round-leaved Sundew.)

Generally distributed, Queen Charlotte Islands; Vanconver Island.

\section{CRASSULACE正 (Orpine Family).}

RHODIOLA.

R. Alaskana Rose.

Queen Charlotte Islands-Dawson Harbour. IN. C. F. Nercombe; Chatla Village, west coast. 
Sents (Stonecrop): Orpine).

s. divergens Wats.

G(rucrally distributed in mountains, Luruaby Island: Queen Charlotte Isliutus ; Vancourer Island.

S. Domglasil Hook.

Iate. Mount Finlayson, V.I., Macoun; Shawnigan Lake, V.I., J. R. Anderson.

S. Oreginum Nintt.

locally distributed, Vancourer Island-Mount Mark; Mount Arrowsmith.

S. frigionm liviberg.

Lowally distributed. Skidegate, Yakoun Lake, Queen Charlotte Islands.

s. spathulifolium Ilook.

(ienerally distributed. Queen Charlotte Islands; Vanconver Island..

S. stenopretalum L'ursh. (Narrow-petalled Stonecrop.)

Locally distributed, Vantouver Island-Victoria. Queen Charlotte Islands.

\section{SAXIFRAGACE正 (Saxifrage Family).}

Borminia (Aconite Saxifrage).

B. cinciunatal Rosendahl \& R̦̣̂tlb.

Locally" distributed, Vinconver Island-Goldstrant : Toluelet.

B. occidentalis Torr \& Gray. (Western Saxifrage.)

Generally distributed, Vaneourer Island.

IIEcemeri (Alum Root).

H. chlorantha Piper.

I,ocally distributed, Queen Charlotte Islands, Dr. C. F. Neucombc; Alberni, Y.I.

H. glabra Willd. (Smooth Alum Root.)

Common. Queen Charlotte Islands; Vancourer Island.

II. mierantha Dougl.

Common. Queen Charlotte Islands; Vaneourer Island.

LePTarmhena (Pear Leaf).

I. amplexifolia (Sternb.) Ser.

Generally distributed in mountains, Vaneouver Island.

Mrtelia (Bishop's Cap; Mitrewort).

M. Breweri Wats.

Locally distributed, Tancouver Island-mountains north of Cowichan Lake.

M. caulescens Nutt.

Rare. Albelni, V.I.. W. R. Carter; Cowichan, V.I. (Glendenning's List, 1918.)

II. ovalis Greene.

Locally distributed, Vaneouver Island-Alberni ; Cowiehan ; Comox; Mount

IBenson; Iienfrew District.

M. pentanchra Ilook.

I. ocally distributed in mountains, Vancourer Island-Momnt Arrowsmith.

PARNASSIA (Grass of Parnassus).

P. fiubriata Koenier. (Fringed Grass of Parnassus.)

Joeally distributed, Queen Charlotte Islands. Vaneourel Island-Mount Arowsmith 'Trail; Clemainus and Koksilah livers. 
[Pundurtures (Mock Orange; Sirringa).

I'. Gordoniaums Liuml.

Locally distributed. southern portion of Vancourer Island-C'amemon Lake. south.

Iines (Currant; Gooseberry).

R. bracteosum I)ougl. (sitiuk Curiant).

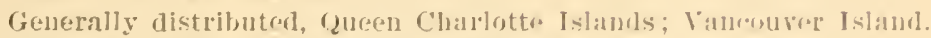

I. divarioatum Dougl. (rommon Gorseluerey).

ficnerally distributed, Vaucouver Island.

I. lacustre (I'ers.) Ioir. (Swamp Gooseberry).

Genrally distributed, Vancouver Island; Queon Charlotte Islands.

R. laxiflorum Pursh.

Iocally distributed, Queren Charlotte Islands (Osgorr, "Nortl Amorican Fauna," No. 21). Vancouver Island-Jiarkley Sound; Lcluelet.

R. Lobbii A. Gray. (Fed-flowrerl (joselorry.)

Common. Southern half of Vancouver Jsland.

R. Lobbii $x$ divaricata.

Mill Hill, Victoria, T.I., Geroge Knight. The first natural bylorid in Jibres discovered in North America. ("Cinadian Field Vaturalist," XXXII., Nov., 1919, 1. 94.)

R. rubrum I. (Iied Currant.)

Common garden currant.

R. sanguineum Pursh. (Red-flowered Currant.)

Generally distributed, southern half of Vancouver Island.

R. setosum Lindl. (Bristly Gonseberry.)

Liare. Strathcona Lark, V.I., J. M. Macoun.

Saxifraga (Saxifrage).

S. odontoloma l'iper.

Locally distributed, west coast Queen Charlotte Islands, Dr. C. F. Veucombe. Vancouver Islaud--Mount Arrowsmith Trail.

S. Bongardi (I'resl.) Pursh.

Locally distributed, Queen Charlotte Islands (Osgord, "North American Fauna," No. 21). Vancouver Island-IIount Arrowsinith Trail; Iount Prevost.

S. bronchialis L. (Common IIonntain Saxifrage.)

Gelerally distributed, mountains, Vancouver Island.

var. austromontana (Wiegand) Pirer.

Generally distributed, mountains, Vancouver Jsland.

var. vespertina (Small) Losendahl.

Iocally distributed, Quren Charlotte Islands, Canoe Pass, W. Spread孔rough.

S. cespitusa L. ('Tufterl Saxifrage.)

Locally ristributed, Queen Charlotte Islands. Vancouver Island- Tellington; Iount Finlayon; T\%ouhalem; Siduey.

S. integrifolia Hrosk.

Generally distributed, southern half of Vancourer Island.

S. Lyallii Engeler. (Iyall's Saxifrage.)

Rare. Mount Arrowsmith, V.I., Kermode \& Carter.

S. Mertensiana Bong. (Syrotted Saxifrage,)

Locally distributed, Queen Charrotte Islands (0sgoror, "North Amerionu Fauna," No, 21). Vaurouver Island-Cameron Lake; Mount Arrowsnith ; Cowichan Iiver.

S. Telsoniana D. Urn.

Locally distributed, Cowichan Lake. T.I. 
SAXIfraga-continuel.

S. Newcombei Small.

Rare. Triangle Island; Queen Charlotte Islands, Dr. C. F. Newcombe.

s. occidentalis Wats.

Generally distributed, Vancourer Island.

S. rufidulal Small.

Generally distributed, Vancourer Island.

S. Tolmiei T. \& G.

Locally distributed in mountains, Vancouver Island-Strathcona Park;

Mount Arrowsmith; Cowichan Lake.

Telmia (Fringe Cup).

T. grandiflora Dougl.

Common. Vancouver Island.

T. parviflora Hook.

Common. Southern half of Vancouver Island.

T. tenella Walp.

Common. Southern end of Vancouver Islaud.

Tiarella (False Mitrewort).

T. laciniata Hook. (Cut-leaved Mitrewort.)

Common. Queen Charlotte Islands. Vancouver Island-Beaufort Range;

Cowicluan; Alberui. Not south of Shawnigan Lake.

T. trifoliatia L. ('Three-leared Mitrewort.)

Common. Queen Charlotte Islands; Tauconver Island.

T. unifoliata Hook. (Simple-leaved Mitrewort.)

Rare. Victoria, Departure Bay, and Goldstream, V.I.; Queen Charlotte Islands, $D r . C . F$. Ycucombe.

TOLMIEA.

T. Menziesii (Pursh.) T. \& G.

Generally distributed, Queen Charlotte Islands; Vancouver Island.

\section{ROSACEE (Rose Family).}

Alchemilla.

A. macrosepala Rydb. ((Lady's Mantle.)

Locally distributed, sonther'n half of Vancourer Island-Sproat Lake Falls, Alberni ; Victoria.

Amelanchuer (June Berry; Service Berry).

A. Cusickii Fernald. (Thick-leaved Service Berry.)

Locally distributed, Cowichan River, V.I.

A. florida Lindl.

Locally distributed, Queen Charlotte Islauds, Dausom. Common, Vanconrel Island.

Aruncus.

A. sylvester Kost. (Goat's Beard.)

Generally distributed, Queen Challotte Islands: Vancouver Island.

Cotoneaster.

r. I'yranconthe (I.) Spach. (Evererees Fire Thorn.)

Cultivated, saauich, Y.I. 
('IAT Eges (Thorn).

('. brevispina Dougl. (Black IIawthoru.)

Locally distributcd, Qucen C'barlotte Islands (Osgood, "Nolth American Fauna." No. 21). Vancouver Island-Comox; Cchucklesit; Victoria ; Alberni.

(:. Oryncentha L. (Euglish Hawthorn.)

Sidney, Y.I., Macoun.

Fragaria (Strawberry).

F. bracteata Heller. (Wood Strawberry.)

Common. Vancourer Island.

F. chiloünsis (L.) Duch.

Common. Queen Charlotte Islands; Vancouver Islanu.

F. crinita Rydb.

Iocally distributed, Sidney, V.I., Macoun.

F. cuneifolia Nutt.

Locally distributed, Vancouver Island-Cowichan. (Glendenning's List, 1918.)

F. Helleri Holzinger.

Locally distributed, Sidney, V.I., Macoun.

Geum (Arens).

G. calthifolium Menzies.

Locally distributed, Queen Charlotte Islauds. (Osgood, "Nortll American Fauna," No. 21).

(r. olegonense Scheutz.

Locally distributed, Sidnes, V.I., Macoun.

G. macrophyllum Willd. (Large-leaved Iellow Arens.)

Common. Queen Charlotte Islands; Tanconver Island.

G. strictum Ait. (Yellow Arens.)

Locally distributed, Vancourer Island-Alberni.

LUTKEA.

L. pectinata (Pursh) Kuntz. (Alpine Spiræa.)

Common. Queen Charlotte Islands; Vancouver Island-in mountaius.

Nuttallia.

N. cerasiformis T. \& G. (Bird Cherry.)

Generally distributed, Vancourel Island.

PHySOCARPUS.

P. opulifolius (L.) Maxim. (Nine-bark.)

Common. Vancourer Island.

I'otextilla (Cinquefoil).

P. albida Rrdb.

Locally distributed, Saanich, V.I., Macoun.

I'. Anserina L. (Silver-weed.)

Common. (Queen Charlotte Islands; Vancourer Island.

I'. biennis Greene.

Vancouvel Island. (Henry's "Flora of Southern British Columbia.")

P. achroa Ricdb.

Locally distributed, Victoria, V.I., Macoun.

P. dissecta I'urch. var. Glaucophylla Wats. (Smooth Cinquefoil.)

Generally distributed in mountains, Vancouver Island. 
POTENTILLA-COntinucd.

['. flabellifolia.

Rare. Strathcona Lark, V.I., J. M. Macoun.

P. glandulosa LindI.

Generally distributed, sonthern half of Vanconrer Island.

P. qraeilis Dougl. (Small ('inquefoil.)

Locally distributed, sonthern end of Vancourer Island-Victoria.

I. monspeliensis L. (Rough (inquefoil.)

Generally distributed, Vancourer Island.

[?. nivea L. (Snows Cinquefoil.)

Rare in mountains, Vancourer Island-IIount Arrowsmith, Flctcher and Anderson.

I'. palustris (L.) Scop. (Min ssh or Purple Cinquefoil.)

Generally distributed, Vancourer Island.

I. recta Linn.

Rare. Union Bay, V.I., Macoun.

P. valida Greene.

Locally distributed. Victoria, V.I.

P. rillosa Pall.

Locally distributed, Queen Charlotte Islands (Osgood, "North American Fauna," No. 21). Vancouver Island-Nootka ; Ucluelet; Mount Arrowsmith; Reufrew District.

I'. Wrangeliana Fisc. \& Meyer.

Mount McDonald and Oik Bas, Victoria, Y.I., Dr. C. F. Newcombe.

Prunuts (Plum or Cherry).

I. Iemissa Nutt. (Choke Cherry.)

Rare. Sonthern end of Vancourer Island.

I. emarginata Dongl. (Wild Cherry.)

Generally distributed, Vancourer Island.

I'rros (Apple; Pear).

I'. diversifolia Bong. (Crab-apple.)

Common. Queen Charlotte Islands; Vancouver Island.

I. occidentalis Wats. (Western Momtain Ash.)

Generally distributed, Vancouver Island.

P. sitchensis (Roem.) Piper. (Alaskan Mountain Ash.)

Generally distributed, Queen Charlotte Islands; Vanconver Island

liosa (Rose).

I. gymuocarpa Nutt.

Common. Vancouver Island.

Ii. nutkana Presl.

Conmon. Queen Charlotte Islands; Vancourer Island.

IR. pisocarpa Gray.

Locally distributed, Vancourer Island-Victoria; Cowichan.

li. rubiginosa L. (Sweetbrier.)

Locally distributed, southern end of Vancouver Island-Victoria.

litib's (Raspberry; Blackberry).

li. arcticus I. (Aretic Bramble.)

Rare. Strathcona Park, V.I., J. JI. Macoun.

R. Chamarmorus Lim. (Cloudberry.)

Locally distributed, Masset, Queen Charlotte Islands, Dr. C. F. Nencombe;

Vancouver Island, near Comox Lake, Iucoun.

R. Id $\alpha u s \mathrm{~L}$.

Locally distributed, Sidnes, Y.I., Iucoun. 
Rubes-Continued,

R. laciniatus Willu. (Erergreen Blacliberry.)

Common. Garden eseape, Vancourer Island.

R. lasiococcus Gray.

Rare. Cowichan Lalke, T.I.

I. leucodermis Dougl. (Black Raspberry.)

Generally distributed, Vancourer Island.

R. macropetalus Dongl. (Trailing Blackberry.)

Common. Vancourel Island.

R. nivalis Doutri.

Rare. Stratheona Park, V.I., J. M. Macom, Comox, V.I.. II. B. Anderson.

I. parvillorus Nitt. ('Thimbleberry.)

Common. Queen Charlotte Islands; Vancouver Islaud.

var. Fraserianus $J$. K. H $\mathrm{enry}$.

Locally distributed, Teluelet, V.I., Geo. Fraser.

I. pedatus suith. (Creeping Raspberiy.)

Generally distrlluted. Queen Charlotte Islands; Vancourer Island.

li. spertabilis I'ursh. (Nialmonberry.)

Common. Queen Charlotte Islands; Vancouver Island.

SANGTISOREA (Burnet).

s. latifolia (Hook.) Coville.

Common. Queen Charlotte Islands. Tanconver Island-Henderson Lake.

S. microcepluala Presl.

Locally distributed, Vancouver Island-Alberni. Yakoun Lake, Q.C.I.

S. occidentalis Nutt.

Locally distributed, Vancourer Island-Quamichan; Sanich.

SPIR玉A.

S. discolor Pursh. (Ocean Spray.)

Common. Vancourer Island.

S. Douglasii Hook. (Hardhack.)

Common. Vancourer Island.

S. Douglasii forma alba.

Locally distributed, Vancouver Island-Cplands, Victoria, Dr. C. F. Tetcombe.

S. Menziesii Hook.

Locally distributed, Vancouver Island-Nootka; Alberni; Renfrew District; Saanieh.

S. salicifolia L.

riare. Coluelet, I.I.

SibBaldia.

S. procumbens L.

Generally distributed in mountains, Vancouver Island.

\section{LEGUMINOSÆ.}

Astragales (Milk Veteh).

1. leucoprsis Torr.

Locally distributed, Nanaimo, T.I., Macoun.

Crtises (Broom).

('. scoparius Link. (Broom.)

Generally distribnted, and spreading where sown, Vancouver Island. 
HosACKiA (Bircl-foot Clover).

H. americana (Nutt.) Piper. (Spanish Clover.)

Locally distributed, southeln end of Vancouvel Island-Esquimalt, Macoun.

H. bicolor Dougl.

Very locally distributed, Harewood, Northfield, Wellington, V.I., Macoun; II. R. Carter.

H. denticulata Drew.

Locally distributed, southern half of Vancouver Island-Alberni; Victoria.

II. gracilis Benth.

Locally distributed, Victoria, V.I., Niss Burris. Now quite plentiful, Oak

Bay, William Head, V.I.

H. parvifiora Benth.

Common. Vancouver Island.

Lathyos (Pea).

L. latifolius L. (Perennial Pea.)

A garden escape, Victoria, V.I.

L. littoralis (Nutt.) Endl. (Shore Pea.)

Locally distributed, west coast, Vancouver Island, sandy shores, Ahousat.

L. maritimus (L.) Bigel. (Beach Pea.)

Common. Queen Charlotte Islands; Vancouver Island.

L. nevadensis Wats.

Rare. Cowichan Lake, T.I., J.R. Anderson.

L. Nuttallii Wats. (Purple I'ea.)

Common. Vancouver Island.

L. palustris L. (Marsh Pea.)

Locally distributed, Vancouver Island-Alberni; Cowichan.

L. panciflorns Fernald.

Locally distributed, Courtenas, T.I., Macoun; Sooke, T.I.

Lepines (Lupine).

L. arboreus Sims.

Garlen escape, Victoria, V.I.

L. bicolor Lindl.

Common, southern end of Tancouver Island.

I. rolumbianus Heller.

Generally distributed, Vancouver Island; Skidegate Sands])it, Q.C.I.

L. laxiflorus Dougl.

Vincouver Islind, Wratson. (Macoun's "Catalogne of Plants.")

L. lepidus Dougl.

Locally distributed, southern end of Vanconver Island-Victoria; Goldstream.

I. littoralis Dougl. (Chinook Licorice.)

Locally distributed, Vanconver Island-Alberni; Comox, Macoun. Rose

Spit, Skidegate, Queen Charlotte Islands, Mr. C. F. Teucombe.

L. micranthus Dougl.

Locally distributed, Vancouver Island-Nanoose; Nanaino.

I. microcarpus Sims.

Iocally distributed, sonthern end of Vaneuver Island-Victoria.

L. nootkatensis Donn.

Common. Queen Charlotte Islands; Vancouver Island.

J. nootkatensis malaskensis Wats.

Jocally distributed, (gueen Charlotte Islands. (Osgood, "North Americrin F'ı una," No. 21.) 
LATIIYRUS-Continued.

L. polyphyllus Lindl.

Generally distributed, southern half of Vancouver Island-Alberni; Vic. toria.

L. sulphureus Dougl.

Locally distributed, Victoria, V.I., Macoun.

Lores.

L. corniculatus L. (Ground Honeysuckle.)

Rare. Vancouver Island-Victoria, Rer. E. L. Grecue.

Medicago (Medic).

M. arabica Huds. (Spotted Medic.)

Locally distributed, Vancouver Island-Victoria.

M. orbicularis (L.) All.

Locally distributed. Vancouver Island-Esquimalt.

II. denticulata Willd.

Locally distributed, Nimaimo, T.I., Macoun.

11. hispida Gaertn. (Bur Clover.)

Localls distributer, Nanaimo, V.I.; Victoria, V.I.

M. lupulina L. (Black Medic.)

Locally distributed, Vancouver Islaud-Victoria; Nanaimo.

M. sativa L. (Alfalfa.)

Common. Escape from eultivation, Vancouver Islanrl.

Melilotus (Sweet Clover; Mililot).

M. albu Desr. (White Sweet Clover.)

Locally distributed, Vancourer Island-Tictoria.

M. Inelica (L.) All.

Locally distributed, Vancouver Island-Nanaimo.

11. officinalis Lam. (Yellow Sweet Clover.)

Locally distributed, Vanconver Island-Victoria; Nanaimo.

Psoralea (P'soralea).

P. physodes Dougl.

Locally distributed, southern half of Vancouver Island-Alberni ; Parks-

ville; Cowichan; Sanich.

Rogixia.

R. Pseudo-Acacia L. (Locust Tree.)

Saanichton, V.I., Macomn.

Trifomua (Clorer; 'Trefoil).

T. agrarium I. (Hop Clovel.)

Generally distributed, Vancouver Island.

T. albopurpureum Torr \& Gray.

Locally distributed, southern end of Vancouver Island-Oak Bay. MI acoun;

William Head.

T. arvense L. (Rabbit-foot Clover.)

Rare. Englishman's River, V.I., I. R. Carter.

T. cyathiferum Lindl. (Fringed Clorel'.)

Rare. Vancouver Island-Mount Benson, Macoun: also reported from Chemainus and Cowichan Rivers.

T. depauperatum Desv. (Low Clorer.)

Generally distributed, southeru half of Vancouver Island. 
TrIFOLIII-C'ontinner.

T. dubium Sibth. (Hop, Trefoil.)

Common. Vancouver Island.

T. fimbriatum Lindl. (Perennial Clover.)

Common. Tancourer Island; Queen Charlotte Islands.

T. fucatum Lindl.

Locally distributed, Cedar Hill, V.I., Fletcher.

T. gracilentum $\mathbf{T}$. \& $\mathbf{G}$.

Locally distributed, Cedar IIill, V.I., Macoun.

T. hybridum L. (Alsilie Clover.)

Locally distributed, Vancourer Island; an escape from cultivation.

T. incaruatum L. (Crimson Clover.)

Locally distributed, Vancouver Island-Victoria; Alberni.

T. medium I. (Mammoth Clover.)

Locally distributed, Vancourer Island. (Hemry's "Flora of Southern British Columbia.")

T. microcephalum Pursh, (Saucer Clover.)

Generally distributed, southern half of Vancouver Island.

T. miclodon H. \& A. (Cup Clover.)

Generally distributed, southern half of Vancourer Island.

T. oliganthum Steud. (Few-flowered Clorer.)

Generally distributed, Vancourer Island.

T. pratense I. (Red Clover.)

Generally distributed. Vancourer Island.

I. procumbens L. (Low Hop Clover.)

Common. Tancouver Island.

T. repens L. (White Clover.)

Common. Tancouver Island.

T. tridentatum Lindl. (Lance Clover.)

Locally distributed, Vancourer Islant-Sidney, Macoun; Victoria.

T. rariegatum Nutt. (Variegated Clover.)

Locally distributed, southern half of Tancourel Island-Sidney ; Nanaimo. Masne Island.

ILEx (Fulze; Gorse).

U. curoparus L.

Locally distributed, southern half of Vancourer Islant-introduced in Victoria.

Vicia (Tetch).

V. americana Muhl, (American Vetch; Pea Vine.)

common. Vancourel Island.

Vir. truncata (Nutt.) Brewer.

Common. Vancouver Island-Albelni; Sidney; Victoria.

$V$. angustifotiu Reich. (Common Vetch.)

C'ommon. Vancourel' Island.

V. Cracer L. (Cow Vetch.)

Locally distributed, southern half of Vancouver Island-Alberni; Telliligton.

V. gigantea Hook. (Till Vetch.)

(Generally distributed, Queen Charlotte Islands; Vancouver Island.

V. hirsuta (L.) S. F. Gray. (Hairy Vetch.)

Locally distributed, southern end of Vancourer Island-Victoria.

V. lathyroikes $\mathrm{L}$.

Iocally distributed, Langford Plains, V.I. 
Vicia-continucl.

T. sutive I. (Spring Vetell.)

Locally distributed, Vancourer Island-Victoria; Nanilino; Sidney.

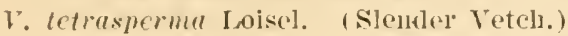

Locally distributed, Sidney, V.I., Ilacoun.

Lixta (Flax).

\section{LINACE IE (Flax Family).}

L. Usitatissimum L. (('ommon Flax.)

Found as an escalle, Vancourer Island.

\section{OXALIDACEE (Wood Sorrel Family).}

Oxalis (Wood Solmel).

O. comiculutu L.

Rare. Nanaimo, V.I.. Macoun.

\section{GERANIACEFE (Geranium Family).}

Erodiua (Storksbill).

E. ricutarium (L.) L'Her. (Hemlork Storlisbill.)

Common. Vancourer Island.

E. moschatum (L.) LiHer.

Locally distributed. Esquimalt Village, V.I., Jucoun.

Geraxium (Geranium; Cranesbill).

G. Bicknellii Britton. (Bicknell's Cranesbill.)

Generally distributed, southern half of Tancouver Island-Alberni;

Nanoose; Sluawigan.

G. carolinianum L. (Carolina Cranesbill.)

Generally distributed, Vancouver Island.

G. dissectum L. (Cut-leaved Cranesbill.)

Generally distributed, Vancouver Island.

G. molle L. (Dove's-foot Cranesbill.)

Common. Vancouver Island.

G. pusillum Burm. f. (Small-flowered Geranium.) Generally distributed, Vancouver Island.

G. Richardsonii F. \& T. (White Geranium.)

Skidegate \& Limestone Island, Q.C.I., I)r. (. F. Tevcombe.

G. Robertianum L. (Red Robin:)

Rare. Vancouver Islank-Comox, Jucoun.

\section{EUPHORBIACEÆ (Spurge Family).}

Elphorbia (Spurge).

E. hirsuta ('Torr.) Wiegand.

Locally distributed, Sproat Lake, V.I., W. R. Carter.

E. Peplus L. (Petty Spurge.)

Locally distributed, Vancourer Island-Yictoria.

E. serpyllifulia Pers.

Locally distributed, Vancouver Island-Victoria.

\section{CALLITRICHACEÆ (Water Starwort Family).}

Callitriche (Water Starwort).

C. Bolanderi Hegelm.

Common. Vancourel Island. 
Callitriche-Continued.

C. hamulata Kuntz.

Vancouver Island. (Henry's "Flora of Southern British Columbia.")

C. heterophylla Pursh.

Generally distributed, Vancouver Island.

\section{EMPETRACER (Crowberry Family).}

EMpetrua (Crowberty).

E. nigrum L.

Common. Queen Charlotte Islands. Vancouver Island, except the southern end--Holberg; Ucluelet; Mount Arrowsmith.

Liminanties.

\section{LIMNANTHACEÆ (False Mermaid Family).}

L. Macounii Trelease.

Rare. Victoria, V.I., Macoun, 1Si5. Not known from any other locality.

Rints (Sumac).

\section{ANACARDIACEÆE (Sumac Family).}

R. diversiloba T. \& (t. (Poison Oak.)

Locally distributed, Sanich Arm, V.I. J. R. Anderson.

\section{CELASTRACE正 (Staff Tree Family).}

Pachistima.

I. myrsinites Raf. (False Box.)

Generally distributed, southern balf of Vancouver Island-Cameron Lake; Saanich.

Acer (Maple).

ACERACEÆ (Maple Family).

A. glabrum Torr. (Smooth IIaple.)

Generally distributed, Vancouver Island.

A. macrophyllum Pursh. (Broad-leaved Maple.)

Common. Vancouver Island.

A. Negundo L. (Cut-leaved Maple.)

Locally distributed, Streets of Nanaimo, V.I.

RHAMNACEÆ (Buckthorn Family).

CEanothus (Red-root; Snowbrush).

C. sanguineus Pursh.

Locally distributed, Vancouver Island-C'ameron Lake: MIalahat.

C. velutinus Dougl. var. levigatus $\mathrm{T}$. \& $\mathrm{G}$.

Locally distributed, IIorne Lake, Y.I., Macoun; Cameron Lake, V.I.

Rimasines (Buckthorn).

Ii. Purshiana DC: (Cascara Sagrada.)

Generally distributed, Vancourer Island, with the exception of the north end.

MALVACEE (Mallow Family).

Malya (Mallow).

31. borealis Wallm.

Locally distributed, Vilncouver Island-Nanaimo, Macoun. 
Malva-Continued.

M. moschata L. (Musk Mallow.)

Locally distributed, Vancouver Island-Siduey.

M. neglecta Wallr.

Locally distributed, Vancouver lsland-Beacon Hil!, Victoria.

11. partifloru I.

Locilly distributed, Foul Bay, Victoria, V.I., Macoun.

M. rotumdifoliu L. (Common Mallow; Cheeses.)

Locally distributed. Vancourer Island-Victoria ; Nanaimo; Sidney ; Tzon-

h:lem; Nootka.

Malvastruar (False Mallow).

M. syliestre (L.)

Locally distributed, Nanaimo. V.I.. Macoun.

Sidalcea (Marslı Hollyhock).

S. IIendersonii Wats.

Generally distributed. sonthern half of Vancouver Island.

HYPERICACEÆ (St. John's-wort. Family).

ELODEA.
E. campanulata Pursh.
Ucluelet, V.I.

HÝPERICU M (St. John's-Wort).

H. anagalloides C. \& S.

Common. Vancouver Island.

I1. canadense L. (Canadian St. John's-mort.)

Locally distributed, Sproat Lake, V.I.

II. perforatum L. (Common St. John's-wort.)

Rare. Koksilah, V.I.; Alberni, V.I., MIs. Cory Wood.

H. Scouleri Ilook. (Sconler's St. John's Wort.)

Common. Vancourer Island.

\section{ELANTINACEE (Waterwort Family).}

Elatise.

E. americana (Pursh) Arn. (Waterwort; Mud Purslane.)

Rare. Alberni, V.I., Jucoun.

ViOLA.

\section{VIOLACEZE (Violet Family).}

V. adunca Smith. (IIooked Violet.)

Common. Queen Charlotte Islands; Vancourer Island.

r. Andersonil Greene.

Locally distributed, Esquimalt, V.I., J. R. Anderson.

r. blanda Willd. (Swett White Violet.)

Bearer Lake. T.I.

V. compacta Greene.

Locally distributed. Shawnigan, Y.I., J. R. Anderson.

r. fulcrata Greene.

Vancouver Island-Cowichan River,J. R. Anderson.

V. glabella Nutt. (Yellow Violet.)

Common. Queen Charlotte Islands; Vancouver Islani.

V. Howellii Gras.

Locally distributed. southern portion of Taneourer Island. 
VHola-remtimed.

V. Langstortlii Fischer.

Locally distributed, Vancourer Island-Shawnigan; Ucluelet; Saanich Peninsula.

v. Macloskeyi Llogd.

Vancouver Island-Prospect Lake.

r. montanensis Rydb.

Localls distributed, Vancourer Island-Saanichtou, Maconn.

V. Nuttallii Pursh. var. priemorsa (Dongl.) Wats. (Nuttall's Violet.)

Locally distributed, southern end of Vancouver Island-Cowichan; Saanich; Victoria.

V. orbiculata Geyer. (Round-leaved Yellow Violet.)

Rare. Strathcona Park, V.I., J. M. Macoun; Mount Benson, V.I., J. R. Anderson.

V. pallens (Banks.) Brainerd. (Northern White Violet.)

Locally distributed, Alberni, V.I., Hemry; Beaver Lake, V.I., J. $R$. Anderson.

V. palustris L. (Marsh Violet.)

Generally distributed, Vancouver Island.

var. leimonia. J. K. Henry.

Vancouver Island. (Henry's "Flora of Sonthern British Columbia.")

v. sempervirens Greene. (Evergreen Yellow Violet.)

Common. Vancouver Island.

$V$. tricolor L. iar. arcensis L. (Lady's-delight.)

Experimental Farm, Sidney, V.I., Macoun.

\section{CACTACE E (Cactus Family).}

Opextia (Ciactus).

O. polyacantha IIaw. rar, borealis Coulter. (Many-spined Opuntia.)

Locally distributed, southern end of Vancourer Island-Cowichan; Gordon Head; Cadboro Bay; Hernando Island, Gult of Georgia.

\section{ELAEAGNACE FE (Oleaster Family).}

SHEPIERDIA.

S. canadeusis (L.) Nitt. (Cauadian Buffalo Berry.)

Generally distributed, Vincouver Island.

\section{LYTHRACE无 (Loosestrife Family).}

LYTHRUM (Loosestrife).

L. Salicaria L. car. tomentosum (IIill) DC. (Spiked or Purple Loosestrife.)

Iare. Alberni, V.I., W. R. Curter.

\section{ONAGRACE E (Evening Primrose Family).}

Boisuevalia.

B. densiflora (Lindl.) Wats.

Locally distributed, Victoria, Wellington, and Qualicum, r.I.

C'ircas (Enchanter's Niglitslade).

C. alpina $\mathrm{L}$.

Common. Quecu Cliarlotte Islands.

C. pacifica Ascols.

Common. Vancouver Island. 
CLARKIA.

('. pulchella l'nush.

Rare. Somenos, Y.I.

Erilomicat (Willow-herb).

i. adenocaulon Haussk. (Northern Willow-herb.)

Common. Vaucouver Island; Triangle Island, Dr. C. F. Neucombe; Queen Charlotte Islands, Dauson.

var. occidentale Trelease.

Common. Vancouver Island.

L. alpinum L. (Apine Willow-herb.)

Locally distributed, Vancourer Island-mountains north of Cowichan

Lake; Strathcona Park; Mount Arrowsmith.

E. anagallidifolium Lam. (I'impernel Willow-herb.)

Locally distributed, Mount Benson and Mount Arrowsmitl, V.I.

E. angustifolium L. (Willow-herb; Fireweed.)

Common. Vancouver Island; Queen Charlotte Islands, Dauson.

E. Franciseanum Barb.

Locally distributed, Vancouver Island-Qualicum, Macoun; Cowjchan. (Glendeming's List, 191S.)

E. glandulosum Lelım.

Locally distributed, Queen Charlotte Islands. (Osgood, "North American Fauna," No. 21.)

E. Halleanum Haussk.

Locally distributed, Nanaimo, V.I.; Mount Donglas ("Cedar Hill"), T.I.

E. Hornemannii Iieich.

Queen Charlotte Islancls, Osgood: Vancourer Island, Macoun.

E. latifolium L. (Water Willow-herb.)

Locally distributed, Renfrew, Y.I., Rosendahl; Cameron Lake, V.I.

E. lutenm Pursh. (Yellow-flowered Willow-herb.)

Iare. Between Buttle and Great Central Lakes, Capt. J. G. Cory Wood.

E. minutum Lindl.

Locally distributed, Iount Benson, V.I., Macoun; Queen Charlotte Islands.

E. palustre $\mathrm{L}$.

Locally distributed, cameron Lake, Y.I.

E. paniculatum Nutt. (I'anicled Willow-herb.)

Common. Vancouver Island.

Godetia.

G. caurina Abrams.

Locally distributed, southern end of Fancouver Island-Thetis Lake; Cowichan.

G. epilubioides Wats.

Locally distributed, southern end of Vancouver Island-Nount Finlasson;

Colwood.

(i. gracilis Piper.

Locally distributed. sonthern end of Vancouver Island.

G. Hispielula Wats.

Locally distributed, Vancouver Island-Nanaimo, Macoun.

LuDVIGIA.

L. palustris (I.) Ell. (Water Purslane.)

Locally distributed, Slroat Lake, Y.I., Macoun; Qnamichan Lake. 
dEnothera (Erening Primrose).

O. biemis L. (Common Evening Primrose.)

Locally distributed, southern portion of Vaucouver Island-Alberni; Victoria.

O. micrantha Hol'n.

Locally distributed, Namaimo, V.I., Macoun.

Spharostigma.

S. contortum (Dougl.) Walp.

Rare. Near Victoria, V.I.

\section{HALORAGIDACEEE (Water Milfoil Family).}

Hirpuris (Bottle Brush).

H. montana Ledeb.

Locally distributed, Strathcona Park, Y.I., J. M. Macom.

H. vulgaris L.

Locally distributed, southern portion of Vancouver Island-Somenos Lake.

Mrriopirluear (Water Milfoil).

M. spicatum I. (Spiked Water Milfoil.)

Generally distributed, Vanconver Island.

M. verticillatum L. (Whorled Water Milfoil.)

Locally distributed, Alberni, V.I.: Shawnigan Lake. V.l.

\section{ARALIACEÆE (Gingseng Family).}

Hedera.

II. helir L. (Common. Iry.)

Common. Escape from cultivation.

Fatsia.

F. horrida (Sm.) B. \& II. (Devil's Club.)

Generally distributed, Queen Charlotte Islands; Vaneouver Island. with the exception of the area around Victoria.

UMBELLIFER王 (Parsley Family).

ANeThuM.

A. gratcolens L. (I)ill.)

Rare. Comox, V.I., Macoun.

ANGELICA.

A. grenuflexa Nutt.

Generally distributed, Vancourer Island.

Antrimiscus (Chervil).

A. sativa $\mathrm{L}$.

Jocally distributed, Nanaimo, V.I.

Caucalis (IIedge Parsley).

C. microcarpa $H$. \& $\Lambda$.

Locally distributed. Mayne Island; Nanaimo, Y.I.; Mount Tzouhalem, Glendenuing.

Carum (Caraway).

C. Carri I.

Locally distributed, Victoria, V.I. 
Carem-rontinlled.

C. Gaindueri Gray.

Common. Southeru half of Vancourer Island-Comox; Alberni; Victoria.

C. oregallum Wats.

Iocally distributed, Vancouver Island-Victoria.

C'icuta (Water Ifemlock).

C. Dunglasii (DC) C. \& R.

Common. Vancouver Island.

C. occidentalis Greene.

Swan Lake, V.I.

C. vagans Greene.

Common. Vancouver Island.

\section{Cellopleurum.}

C. Gmelini (DC) Ledeb.

Locally distributed, Queen Charlotte Islands; Vancouver Island. (Thickets near tidal waters, Tancourer Island to Alaska.)

C. longipes C. \& R.

Locally distributed, Vancourer Island. (Henry"s "Flora of Sonthern British Columbia.")

Coxioselixum (Hemlock Parsley).

C. Gmelini C. \& R.

Locally distributed, Queen Charlotte Islands. Vancouver Island-Alberni; Sidney.

C. pacificum Wats.

Locally distributed, Barkley Sound, Y.I., Macoun.

Conien.

C. muculutum I. (Poison Hemlock.)

Lucally distributed, southern end of Vancouver Island-Victoria.

DAicl's (Carrot).

D. carota L. (Cultivated Carrot.)

Locally distributed, Vancouver Island. Escape from cultivation.

D. pusillus Michx. (IVild Carrot.)

Locally distributed, southern half of Vancouver Island-Alberni; Strathcona Park; Parksville.

Erymgiual (Ersngo).

E. articulatum Hook.

Locally distributed, Victoria, V.I.

Fexiculum.

F. vulgare Hill.

Garden escape. Locally distributed, Tancouver Island.

Glehxia.

G. littoralis (Gray) Schmidt.

Locally distributed, Queen Charlotte Islands. Vancouver Island-Tictoria, Macoun.

Heracleua (Cow Parsnip).

H. lanatum Michx.

Common. Queen Charlotte Islands; Vancouver Island. 
Hydocotyle (Water Pennyort).

H. ramunculoides L.f.

Iare. Vancourer Island-Nanaimo, Macom.

LEPTOTENIA.

L. dissecta Nutt.

Locally distributed, southern end of Vancouver Island-in the vicinity of Victoria, Macom!.

Ligusticuat (Lovage).

L. scothicum L.

Locally distributed, ocean coast, Vancouver Island; Queen Charlotte Islands.

L. vulgare Iinn.

Introduced, Victuria, V.I.

LIL EOPSIS.

L. occidentalis (. \& R.

Common. Tancourel Island in most saline marshes-Comox; Alberni; Nanaimo.

Loyatium (Hog Fennel).

L. macrocarpum (N̈utt.) C. \& R.

Mount Finlayson, T.I., 1916. Dr. C. F. Nencombe.

L. Martindalei var. angustatum C. \& R.

Locally distributed in monntains, southern half of Vancouver Island-

Mount Arrowsmith: Henderson Lake.

L. nndicaule Pursh. (Indian Consumption Plant.

Locally distributed, southern half of Vanconver Island-Alberni; Wellington; Victoria.

L. triternatum C. \& R.

Rare. Tzonhalem Monntain. Y.I., Glendenning.

L. utriculatum Nutt.

Common. Southern half of Vancourer Island-Alberni ; Wellington; Victoria.

Oinantife (Water Parsley).

O. sarmentosa Presi.

Common. Sonthern half of Vancourer Island-Comox; Alberni ; Shawnigan; Sidner. Queen Charlotte 1slands, Dr. C. F. Nercombe.

Osmorrinza (Sweet Cicely).

o. brevipes.

Sanuicli Peninsula, Macomm.

O. divaricata Nutt. (Western Sreet Cicels.)

Common. Queen Charlotte Islands; Vancouver Island.

O. Leibergii C. \& R.

Common. Vancourer Island.

O. Jurpurea C. \& Ii.

Rare. Mount Arrowsmith, Y.I., Kermode and Carter; Queen Charlotte Islands, Dr. C. F. Newcombe.

Pastinaca (I'ilrsilin).

P. satira $\mathrm{I}$.

Common. Garden escape, Vincouver Island. 


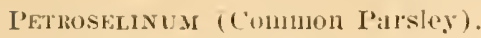

I. hortense IIofin.

Gablen escape. Ioeally distributed. Vanconver Island.

S.IXTctid (Sanicle).

S. allotopoides II. \& A.

Locally distributed, sonthern end of Vancourer Island-Victoria; Queen Charlotte Islands.

s. bipinnatifida Dougl.

Locally distributed, southern end of Vancouver Island-Cowichan; Vietoria.

s. Howellii C. \& R.

Generally distributed, sonther'n balf of Vancouver Island.

S. marilandical Linn. (Black Snakeroot.)

Vanconver Island, Macoun. (Piper \& Beattie's "Flora of the North-west ('oast.")

S. Menziesii Hook.

Common. Southelu halt of Vincouver Island-Comox; Alberni : Yictoria.

S. septentrionalis Greene.

Locally distributed, southern half of Vancouver Island-Alberni ; Trellington.

Sium (Water Parsnip).

S. suave Walt.

Common. Yancourel Islant.

Corsus (Dogwood).

\section{CORNACEE (Dogwood Family).}

C. canadensis. (Bunchbery.)

Generally distributed, Vancouver Island.

C. Nuttallif Audubon. (Flowering Dogwood.)

Common. Southern half of Vancouver Island.

C. stolonifera Michx. (Red-osier Dogwood.)

Common. Tancouver Island; Queen Charlotte Islands.

\section{ERICACEÆ (Heath Family).}

ALLOTROPA.

A. virgata T. \& G. (Striped Allotropa; Deril's Wand.)

Vanconver Island-Alberni; Comox; Wellington; Cowichan; Nanaimo: Malabat; Nitinat.

ANDROMEDA.

A. Polifolia L. (Wild Rosemary.)

Masset, Q.C.I.; Vancourer Island. (Henry"s "Flora of Southern British Columbia.")

Arbetes.

A. Menziesii Pursh. (Arbutus.)

Common throughont the southern half of Vancouver Island.

Arctostaphyos (Bearberry).

A. columbiana Piper. (Manzanita.)

This is the common northern form that has usually been referred to A. tomentosa LindI. Generally distributed, southern half of Vancourer Island-Beaufort Range; Cameron Lake; Cowichan; Wellington; Malahat. 
Arctostaphirlos-Continued.

A. media Greene.

Locally distributed, Vancouver Island, J. M. Macoun.

A. Cra-ursi (L.) Spreng. (Kinnikinick.)

Common. Queen Charlotte Islands; Vancouver Island.

Cassiope (Moss Heather).

C. Mertensiana (Bong.) D. Don. (White-flowered Moss Heather.)

Common in mountains, Vancouver Island; Queen Clarlotte Islands, osgood.

C. Stelleriana (Pall.) DC.

Queen Charlotte Islands, Osgood.

\section{CHIMAPHILA.}

C. Menziesii (R. Pr.) Spreng. (Menzie's Wintergreen.)

Generally distributed, southern half of Vancourer Island.

C. umbellata (L.) Nutt. (Pipsissewa: Prince's Pine.)

Common. Southern half of Vancourer Island.

\section{Cladothamnes.}

C. pyrolæeflorus Bong, (Copper Bush.)

Locally distributed in mountains, Queen Charlotte Islands. Vancourel Islant-Cowichan Lake; Mount Arrowsmith.

Gavltieria.

G. oratifolia Gray. (Western Teaberry.)

lount Arrowsmith, Vancourer Island.

G. shallon Pursh. (Salal.)

Common. Queen Charlotte Islands; Vancouver Island.

KaLmia (Sheep Laurel).

K. polifolia Wang. (Swanı Laurel.)

Generally distributed. Queen Charlotte Islands; Vanconver Island.

K. polifolia mierophyllia (Hook.) Piper.

Generally distributed, Vancouver Island.

Ledum (Labrador Tea).

I. gromlandicum Oeder.

Generally distributed, Queen Charlotte Islands: Vancoirer Island.

LOISELEURIA.

L. procumbens (L.) Desr. (Alpine; Trailing Azalea.)

Locally distributed, Queen Charlotte Islands (Osgood, "North American Fama," No. 21) ; Banks Island, Mcnzies.

MIEXZIESIA.

II. ferruginea Smith.

Queen Charlotte Islands, Dawson. Vancouver Island-Renfrew District; Cowichan Lake; Nount Arrowsmith; Henderson Lake.

Moneses.

1. uniflora (L.) Gray. (Single Delight.)

Locally distributed, Queen Charlotte Islands (Osgood, "North American Famna," No. 21). Vancouver Island; Ilolberg; Cameron Lake; Nitinat. 
Moxotrol's (Pinesilu).

M. muitlora I. (Indian Piue.)

Common. Vancouver Istand.

M. brevis small. (I'imesap.)

Ialle. Leatufort Range, Alberni, Y.I., W. R. Carter.

M. IIylopitys L. (I'inesap.)

Generally distributed. Queen Charlotte Islands; Vancouver Island.

II. fimbriata Glay. (I'iuesaju.)

Rale. Mount Arrowsmith, V.I., Macoun.

Newierrya.

N. congesta (Gray) Torr.

liare. I'ort IRenfrew. V.I., August 1st, 1902, Rosondahl; Alberni, V.I., IV. R. curter; J. F. Bledsoe.

P'IYLlodoce (Mountain Heatli).

F. empetriformis (Smith) I). Don. (Pink-flowered Momntain IIeath.)

Common in mountains. Vaucourer Island.

I'. glanduliflora (Hook.) (ov. (Tellowish Mountain Ifeath.)

Locally distributer in mountains, Vaucouver Island-Nount Arowsmith.

Queen ('harlotte Islauds. (Osgood, "North American Fauna," No. 21.)

PLECRicospora.

P. fiubriolata Gray.

Beaufort Range, above Horne Lake, V.I., IV. R. Carter.

Pterospora.

I. andromedea Nutt. (I'ine-rlroys.)

Locally distribnterl, sontlem half of Vancourer Island-Alberni; Cameron Lake; Malahat; Sidney.

PYrola (Tintergreen).

P. ajhylla Smith. (Leafless Wintergreen.)

Locally distributed, Vancourer Island-Cameron Lake; Mount Benson; Cowichan.

I'. bricteata Hook.

Common. Vancourer Island.

I. (llorantha Swartz. (Greenisli-flowered Wintergleen.)

Locally distributed. Vanconver Island-Alberni; Cowichan; Comox.

I. elata Nutt. (Bog Wintergreen,)

Generally distributed, Vancourer Islane?.

P. elliptica Nutt. (Shin Leaf.)

Rare. Vancouver Island. (Henry"s "Flor'a of Sonther"n British Columbia.")

P. picta Smith. (Spotted Pryola.)

Locally distributed, southern half of Vancourer Island-Alberni; Mount Benson; Cowichan; Malahat.

P. picta var. dentata (Smith) Pijer.

Locally distributed, Vancouver Island-Alberni ; Mount Bensou.

P. picta var. integra A. Gras.

Vanconver Island-Aberni, V.I., Mrs. Cory Wood.

I. secunda L. (One-sided Wintergreen.)

Common. Queen Charlotte Islands; Vancourer Island. 


\section{RHODOUENDRON.}

R. albiflorum Ifook. (White Mountain Rhododendron.)

Generally distributed in mountains, sonthern half of Vancourer Island.

Taccinius (Huckleberry; Blueberry; Bilbery; Cranberry).

V. Alaskaensis Howell.

Rare. Stratheona Park, V.I., J. M. Macoun.

V. deliciosum Piper.

Strathcona Park, V.I., J. M. Macomn.

Y. ciesplitosum Michx. (Dwarf Bilbery.)

Generally distributed, Queen Charlotte Islands; Vancouver Island.

V. macrophyllum (Hook.) Piper.

Generally distributed, Queen Charlotte Islands; Vancourer Island.

V. occidentale Gras.

Mount Mark, V.I., Macoun.

Y. ovalifolium Sm. (Tall or Oval-leaved Bilberry.)

Generally distributed, Queen Charlotte Islands; Vancouver Island.

x parvifolium.

Locally distributed, Strathcona Park, V.I., J. M. Macoun. .

V. ovatum I'ursh. (Evergreen Hucklberry.)

Locally distributed, coast of Vancouver Island; north point of Texada

Island. Occasionally found inland on Vancouver Island-Alberni; Sproat Lake, W. R. ('arter.

V. Oxycoccus var. intermedium A. Gray. (Bog Cranberry.)

Generally distributed, Queen Charlotte Islands; Vancourer Islaud.

V. parvifolium smitl. (Red Huckleberry.)

Common. Queen Charlotte Islands; Vanconver Island.

V. uliginosum L. (Bog Bilberry.)

Locally distributed, Queen Charlotte Islands (Osgood, "North American Famna," No. 21) ; Triangle Island. Vancouver Island-Cowichan.

V. Vitis-Idaea L. ( Rock Cranberry.)

Locally distributed, Ucluelet, V.I.; Queen Charlotte Islands.

\section{PLUMBAGINACE巴 (Leadwort Family).}

Statice.

S. Armeria L. (Sea Lavender; Thrift.)

Common along the coast of Vanconver Island.

\section{PRIMULACE王 (Primrose Family).}

Anagallis (Pimpermel).

A. arrensis L. (Poor Man's Weatherglass.)

Locally distributed, Sidney. Y.I., Macomn.

Centunculus (Chaffweed).

C. minimus $\mathbf{L}$.

Iocally distributed, Vancouver Island-Sproat Lake; Sidney, Maconn.

Dodecatineor (Shooting Star).

D. frigidum Hook.

IRare. Mount Arrowsmith, Macoun; Strathcona Park, V.I., J. M. Macomn;

Limestone Island; Queen Charlotte Islands, D). C. F. Neucombe.

D. Latifolium (IIook.) Piper.

Generally distributerl, Vancouver Islanel. 
DODECATILOX-Continucd.

D. pauciflorm (Durand) Greene.

Common. Vancouver Island.

D. tetrandrum Suksilort'.

Teluelet, $\mathrm{V}: \mathrm{I}$.

D. viriparmu Greene.

Locally distributed, Queen Charlotte IsIands, (Osgond. "North American

Fauna," No. 21.)

(ilatx (Sea Milkwort).

(i. maritima L.

Common, Queen Clarlotte Islands; Vaucouver Island,

Lrimacila (Loosestrife).

I. terestivis (I.) IBSP. (Bulb-bearing Loosestrife.)

Locally distributed, Ucluelet, T.I.

I. thrrsiflora I. (Tufted Loosestrife.)

Locally distributed, southern end of Vancouver Island-Comichan; Bearer

Lake.

Trimatals (Chickweed Wintergreen).

T. arctica Fisch. (Northern Star Flower.)

Locally distributed, Tasu Harbour, Queen CInarlotte Islands, Dr. r. F'. Teucombe. Tanconver Island-Renfew District; Alberui ; Lcluelet; Cowichan; Mount Finlayson.

T. Iatifolia Hook. (Star Flower.)

Common. Queen Charlotte Islauds; Vanconver Island.

\section{GENTIANACE $\mathbb{E}$ (Gentian Family).}

Cextauriua (Centauy).

C. centaurium I.

Locally distributed. Victoria. V.I.

Gextiana (Gentian).

G. acuta Michx. (Northern Gentian.)

Generally distributed, Queen Charlotte Islands; Tanconvel Island.

G. Douglasiana Bong. (Douglas' Gentian.)

Locally distributed, Vancomver Island-Renfrew District, Rosendahl:

Ecluelet. Queen Charlotte Islands, Fort MeLonghlin. Milbank Sound. Dr. Tolmie.

G. platyphylla Griseb.

Rare. Yakoun Lake, Queen Charlotte Islands, Dr. C. F. Teucombe.

G. propinqua Richards.

Cameron Lake, Y.I., T. R. Carter.

G. sceptrum Griseb. (Swamp Gentian.)

Generally distributed, Vancouver Island.

Mexianties (Buckbean).

M. crista-galli Menzies.

Locally distributed, Renfrew District, T.I., Rosendahl; Queen CharIotte Islands, $D r . C$. F. Netcombe.

II. trifoliata L.

Generally distributed, Queen Charlotte Islands; Tancourer Island. 


\section{APOCYNACE E (Dogbane Family).}

Apocrivu (Doghane).

A. androsiemifolinm L. (Spreading Dogbane.)

Generally distributed, southern half of Vaucouver Island.

A. camnabinum $\mathrm{I}_{\text {. }}$ (Indiau Hemp).)

Locally distributed. Cameron Lake, V.I., W. R. Carter; Cowichan River.

Vrinca (Periwinkle).

V. minor L.

Garden escape, Victoria, Y.I.

\section{CONVOLVULACE $Æ$ (Morning Glory Family).}

Convolvulus (Bindweed).

C, aremsis L. (Small Bindweed.)

Locally distributed, Vancouver Island-Victoria; Nanaimo; Cowichan.

C. sepium L. (Hedge or Great Bindweed.)

Locally distributed, Tancouver Island-Victoria ; Nanaimo.

C. Soldanella L.

Locally distributed, Savars Island. Dawson; Pachena Bay, V.I.

Cuscuta (Dodder).

C. arrensis Beyrich.

Locally distributed, Vancouver Island-Cobble Hill; Cowichan.

(. Elithymum Murl.

Locally distributed, Mayne Is]and, Mupoun.

(*. Salina Engelm. (Salt-marsh Dodder.)

Localle distributed, Vancouver Island-Alberni; Victoria.

C. squamigera Piper.

Locally distributed, Sidney, V.I., Macoun.

Collomia.

\section{POLEMONIACEE (Phlox Family).}

C. grandiflora Dougl.

Generally distributed, southern half of Vincouver Island-Comox;

Albelui; Parksville; Cowichan; Victoria.

C. heterophylla Hook.

common. Southern half of Vancouver Is]and.

(. linearis (Nutt.) Gray. (Narow-leared Pllox.)

Southern half of Pancouver Island; Mount Benson; Wellington.

Gillia.

G. achillerefolia Benth.

Locally distributed, Vancourer Island-Shawnigan.

(x. bicolor (Nutt.) Pipex.

Common. Southern half of Vancouver' Is]and.

G. capilala Hook.

Locally distributed, sonthern end of Vanconver Island-Victoria; Shaw-

nigan Lake; Malahat.

G. gracilis lougl.

Common. Sonthern half of Vancouver Island.

G. intertexta Stend.

Mount Douglas (" ('edar IIill"), V.I.

G. squarrosil (Escell.) II. \& A.

Locally distributed, sonthern end of Vancouver Island-Cowichan; Victoria. 
PIILOX.

P. diff̈usil Hook. (Douglas Phlox.)

Common in mountalus, Vancourer Island.

Polemonium (Greek Valerian).

P. elegans Greene.

Locally distributed, Inuncan. Y.1.. Mrs. II adken; Queen Chitrlotte Islands, Dr. C. I. Yercembe.

1. micrintlum bentl.

Rilre. Near Victoria, V.I., Fletcher.

\section{HYDROPHYLLACEE (Waterleaf Family).}

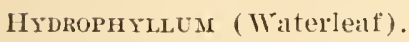

H. tenuipes Ileller.

Locilly distributed. Vincourer Island-Cameron Lake; Goldstream; Sooke River.

Nesrophila (Grove-lover).

x. micrantha Eastwood.

Locally distributed, Vancourer Island-Victoria; ('ameron Lake.

x. parriflora Dougl.

Common. Southern half of Tancourer Island.

న. pedunculata Dongl.

Locally distributed, Vanconver Island-Cowichan (Glendenning's List, 1918) ; Observatury IIIll.

x. sepulta Parish.

Locally distributed. southern half of Vancourer Island-Ninoose; Cowichan; Victoria.

Phacelis.

P. heterophylla Pursh.

Locally distributed. Cowichan Lake, Y.I.; Mount Arrowsmith, Y.I,

I. hispida Gria.

Iare. Nanaimo, Y.I., Ifucoun; introduced from California.

P. linearis (Pursh.) Holzinger.

Locally distributed, Galiano Island, Jucoun.

I. sericeal Gray.

Rare. Vaneonver Islind-Dount Arrowsmith. MIncoun.

Iomanzolfia (Mist Maidens).

R. sitchensis Bong.

Locally distributed. Queen ('harlotte Islands, I)r. C. F. Trucombe. Vancouver Island-Cimeron Lake; Mount Arrowsmith.

R. unalaschkensis Cham.

Locally distributed, Albert Head, V.I., Dr. C. F. Yrucombe: ['cluelet, T.I., Iracoun.

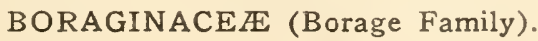

Allocaria.

A. californiea (DC) Greene.

Generally distributed, southern half of Vancourer Island-Alberni; Nanaimo; Cowichan; Saanich ; Victoria. 
Allocaria-C'Ontilluct.

1. divaricata Piper splo nov.

Trpe, T.S. National Herbarium, collected on exposed rocks on the seashore, Victoria, V.I., by Wm. Palmer. Probably taking the place of what we have known as A. plebeja (Cham.) Greene, which is an Alaskan species.

A. media Piper sp. nov.

Generally distributed, sonthern half of Vancouver Island-Alberni ; Cowichan Lake, Roscudahl; Victoria. 'Type, U.S. National Herbarium, collected Port Angeles, Wash. This appears without doubt our common form. Under a recent study of the Allocarya by Professor C. V. Piper, A. chorisiana (Cham.) Greene is eliminated from our list, and the specinens from Mount, Douglas ("Cedar IIill"), Victoria, V.I., collected by Macoun are leferled to here as $A$. media.

A. Scouleri (DC) H. \& A.

Locally distribnted, southern half of Tancouver Island-Northfield; Nanaimo.

Ausinckia.

A. barbata Greene.

Locally distributel, Cameron Lake, V.I.

A. intermedia F. \& $\mathrm{M}$.

Generally distributed, Vanconver Island.

A. lycopsoides Lehm.

Locally distributed, Vancouver Island-Parksville; Cowichan; Sidney;

Texada Island. Queen Charlotte Islands, Dr. C. F. Neuęombe.

Borago.

B. officinailis L. (Borage.)

Rare. William Head, Victoria, V.I., Miss Barbura Cox.

C'RYPTANTHA.

C. ambigua (A. Gray) Greene.

Nanaimo, T.J., Macoun.

LAPPLLA (Stickseed).

L. cupulata (A. (xray) Rrab.

Vicinity of Victoria, V.I., Fleteher. (Maconn's "Catalogue of Plants.")

L. echinata Gil.

Locally distributed, Cowichan, V.I.

L. occidentalis (Wats.) Rrab.

Lacally distributed, Victoria, V.I., Macomn.

Mertensia (Lmogrot).

M. maritima (J.) S. F. Gray.

Queen Clislotte Islands, Dursom.

Mrosotis (Forget-me-not; Scorpion Grass).

M. arensis (L.) Ilill. (Field Forget-me-not.)

Generally distributed, southeru half of Yancouver Island-Alberui ; Comox; Cowichan: Victoria.

11. laxa Lothm. (Small Forget-me-not.)

frenerally distributed, sonthern lialf of Vanconver Island-Alberni;

Nanorse; Nillainlo; Elk Lake.

I. macrosperma (Engelu.) Fernald. (Wnite Forget-me-not.)

lare. Vir.inity of Victoria, V.I., Macoun: Mount Donglas ("Cedar IIill "), Y.I., Fleteher; cowichan River, I'.I., Glemleming. 
Mrosotis-c'ontinued.

1. scorpioides L. (Forget-me-not.)

Albermi, V.I.

1. rersiculor (Pers.) Sm. (Yellow and Blue Scorlion Grass.)

Generally distributed, southern end of Vaucouver Island-Sidney; Esqui-

malt. Mayne Islancl.

\section{VERBENACE无 (Vervain Family).}

Verbexa (Terrain).

V. hastata L. (Blue Verrain; Wild Myssop.)

Rare. Vancourer Island-Victoria; Alberni. Macoun.

LAMIUM.

\section{LABIAT $\mathbb{E}$ (Mint Family).}

L. amplexicanle L. (Henbit; Dead Nettle.)

Locally distributel, Comox and Saanich, T.I.

Lrcoits (Bugle Weed).

L. americanus Muhl. (C'nt-leaved Water Horehound.)

Common. Vancourer Island.

L. uniflorus Michx. (Northern Bugle Weed.)

Generally distributed, Vancouver Island.

Markebium (Holehound.)

II. vulgare L. (Thite or Common Horehound.)

Locally distributed, southern half of Vancouver Island-Wellington; Victoria.

Mextua (Mint).

I. arvensis L. var. canadensis (L.) Briquet. (Field Mrint.)

Common. Vancouver Island.

II. canadensis L. var. glabrata Benth.

Generally distributed, Vancouver Island-Sproat Lake, Macoun.

var. lanata Piper. (Wild Mint.)

Common. Vancouver Island.

1I. pipcrita L. (Peppermint.)

Locally distributed. Sidney and Comox, V.I.. Wacoum.

M. spicata L. (Spearmint.)

rancouver Island-Sidney, Macoun.

M. riridis $\mathrm{L}$.

Locally distributed, Vancourer Island-Victoria.

Mí RoMeria.

11. Chamissonis (Benth.) Greene. (Yerba Buena.)

Common. Tancouver Island.

Nepeta (Cat Mint).

I. Caturia L. (Catnip.)

Locally distributed, Vacnourer Island-Cowichan Bay; Victoria.

X. hederacea (L.) Trev. (Ground Iry.)

Locally distributed, Vanconver Island-Sidney; Wellington; Victoria.

Pruxella (Self-heal).

P. rulgaris L. (Self-heal.)

Common. Vanconver Isimd, Queen Charlotte Islanrls. 
Prunella-Continued.

forma alba.

Locally distributed, Alberni and Fitzgerald, Y.I., W. R. Carter.

forma erubescens J. K. Henry.

Locally distributed, C:meron Lake, V.I.

Scétellaria (Skulleap).

S. angustifolia Pursh. (Narrow-leared Skullap.)

Victoria, Y.I., Macoun.

S. galericulata L. (Marsh Sliulleap.)

Common. Vancourel Island.

S. lateriflora I. (Mad Dog; Iilue Skulleap.)

Locally distributed, Vaucouver Island-Alberni ; Cowichan ; Iaslam Creek.

Stacirs (IIe(Ige Nettle).

S. ciliata Dougl.

Common. Vancouver lsland.

S. ciliatar var. pubeus (Gray) Heller.

Locally distributed, ast coast of Queen Clarlotte Islands, Dawson.

Vancouver Island-Icluelet.

\section{SOLANACE⿸ (Potato Family).}

Solanum (Nightshade).

S. nigrmm L.' (Deadly Niglitshade.)

Locally distributed, Vanconver Island-Wellington; Victoria.

s. nigrum villosmin Lam.

Ju(ally distributed, Vancourel Island-romox: Victorial.

\section{SCROPHULARIACEE (Figwort Family).}

Antiriminga (Smapdragon).

A. Orontium L. (Lesser Snap(Iragon.)

Garten escape, Vancourer Island-Saanich.

Castilleja (Scarlet Paint-brush).

C. angustifolia Nutt, var. Bradburii Feruald.

Generally distributed, southern half of Vancouver Island-Alberni;

Nootka; Wellington; Saanich.

(C. Dixoni Fernald.

Locally distributed, Alberni, V.I.; Triangle Island.

C. levisecta Greenman. (Yellow Paint Brush.)

Locally distributed, southern end of Vancourer Island-Sidney; Victoria.

C. niniata I ougl. (Red Paint Brush.)

(')mmon. Vameouver Island; Queen Charlotte Islands.

C. pallirla (L.) Spreng. (White Paint Brush.)

Locally distributed, (2ucen Clazrlotte Islands, Dateson. Yancouver Island

-Alberni; Tchucklesit.

(.. palriflora Rong.

Locally distributed, Queen Charlotte Islands, manson.

C. Phexifolia Iiydb.

Locally distributed, ('owichin Lake, I.I.

Cinelone (Turtleheid).

(.. nemorosai Dongl.

Locally distributed, Vancouver Island-Renfrew, Rosendahl; Cameron Like; Cowi(chin Jake. 


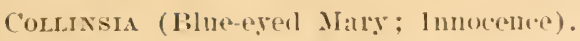

C. gramblifluran Dourel.

Commen. Vanconver Islanul.

rar. lussillat ris:ay.

Common. Vanconvel Islanil.

C. tenellar Inongl.

Generally distributed. Vanconver Island; Queen C'harlotte Islands, Osgond.

Digitalis (Foxglove).

D. purpuren L. (Puple Foxglove.)

Lacally distributed. Vancourer Island-Alberni.

Gratiola (Herlge Hyssop).

G. ebracteata Bentli.

Locally distributed, Vanconver Island-Sproat River: Alberni: Victoria.

G. rirginiana L. (Clammy Hedge Iyssop.)

Iocally distributed. Vancourer Island-Sproat Lake; Alherui ; Comox; Cowichan: Victoria.

Lisoselia (Mndwort).

L. tenuifolia (Tolf) Pers.

Iocally distributed. Alberni. V.I. ; romox, V.I.

IAxAria (Toadflax).

I. camadensis (I.) Dumont. (Wild Toadflax.)

Rare. Departure Bay. V.I.; Isquimalt, V.T.

L. Cymbalaria $\mathbf{I}$.

Locally distribnted. Vancourer Island-garden escapre, Nanajmo.

L. rmlgaris Ilill. (Butter \& Egas.)

Locally distributed. Vanconver Island-Nberni ; Cameron Lalie: Namaimo.

Melampyou (Cow Wheat).

M. lineare Lam. (Narrow-leared Cow Wheat.)

Iocally distributed. Vancouver Island-Cameron Lake: Englishman's River.

Mrutct's (Monkes Flower).

M. alpinus (Gray) I'iler. (Alpine Monkey Flower.)

Locally distribnted. Mount Arrowsmith. Y.I., Fermole and C'arter.

I. alsinoides Dougl.

Comsuon. Vancourer Island.

I. grandiflorns Iowell.

Locilly distributed. Sidney. V.I.. Jarmm.

II. Langsdorffii Dom.

Common. Vancourer Island; Qneen Charlotte Islands.

var. platyphyllus Greene. (Langsdorff's Yellow Monkey Flower.) Nootka.

V.I.; Trelnelet, I'I.

M. Lewisỉi Pursh. (lied Monkey Flower.)

Locally distributed, Cowichan Lake, V.I.; Henderson Lake. V.I.

I. moschatus Dongl. (Musk Flower.)

Generally distributed, Vancourer Island.

II. uasutus Greene.

Common. Vancourer Island.

II. pilosus (Benth.) Wats.

Locally distributed, Sman Lake. V.I. 
Orthocarpus (Lesser Paint-brush).

O. attenuatus Gray.

Locally distributed, southern end of Vancouver Island, vicinity of Victoria.

O. bracteosus Benth.

Locally distributed, southern end of Tancouver Island-Victoria; Sidney.

O. castilleoides Benth.

Locally distributed, southern half of Vanconver Island-Alberni ; Victoria ; Sidney.

O. erianthus Benth.

Locally distributed, southern end of Vancourer Island, vicinity of Victoria.

O. faucibarbatus Gray.

Locally distributed, Vancouver Island-Tictoria.

O. hispidus Benth.

Locally distributed, southern end of Vanconver Island-Cj]lands, Victoria, Macoun.

O. pusillus Benth.

Generally distributed, sonthern half of Tancouver Island-Alberni; Wel-

lington; Cowichan; Victoria.

Pedicularis (Lousemort).

P. lanata Willd.

Locally distributed, Queen Charlotte Islands.

P. pedicellata Bunge.

Locally distributed, Queen Charlotte Islands. "Appears to be identical with P. ornithorhyncha Benth." (C. V. Piper.)

P. ornithorhyncha Benth.

Mount Arrowsmith, V.I., W. R. Carter. (Type locality, Mount Rainier.)

P. racemosa Hook. (White Lousewort.)

Generally distributed, Tancouver Island.

Pentstemon (Beard-tongue).

P. diffusus Dougl.

Loeally distributed, southern half of Vancouver Island-Beaufort Range;

Alberni; Cameron Iake; Cowichan Lake.

I'. Menziesii Iook.

Common in mountains, Tancouver Island-near shore, Nootka Island and Effingham Inlet, Barkley Sound.

Rimnanthos (Yellow Rattle).

1R. Crista-galli $\mathrm{I}$.

liare. Vancouver Island-Tnion Bay; Cedar Hill, Macoun. Common, Masset, Qucen Charlotte Islands, Dauson.

SCropitularia (Figwort).

S. californica Cham.

Locally distributed, west coast of Vancourer Island-Uchucklesit; Clayoquot; Alberni.

Yerbascum (Mullein).

V. Blattaria L. (Moth Mu!lein.)

Comox, V.I., W. B. Anderson.

Verotica (speedwell).

V. alpina L. (Alpine Specdwell.)

Common in momtains, Queen Charlotte Islands; Vancouver Island. 
Venoxica-Continuct.

V. americaua Schwein. (Brooklime.)

Common. Queen Charlotte Islands; Vancouver Islant.

r. arensis I. (Corn sieedwell.)

Common. Vancouver Island.

V. peregrima L. (Neckweed.)

Generally distrihuted, Vaneourer Island.

V. serpyllifolia L. (Thyme-Ieaved Speedwell.)

Common. Vancouver Island.

V. scutellata L. (Marsh Speedwell.)

Common. Vincouvel Island.

var. villosi Schum,

Locally distributed. Sproat Iake, V.I., IIcmry.

V. Tournefortii C. C. Gmel.

Locally distributed, southern end of Tanconver Island-Saanichton, Itcoun ; Victoria.

\section{LENTIBULARIACEÆ (Bladderwort Family).}

Pixgeticlea (Butterwort).

P. vulgaris L. (Common Butterwort or Bog Violet.)

Generally distributed, chiefly in wet mountain marshes in the central and northern portions of Vancouver Island. On Nootka Island it appears almost at sea-lerel. Queen Charlotte Islands.

LTRICUlaria (Bladderivort).

U. intermedia Hasne. (Flat-leaved Bladderwort.)

Locally distributed, Icluelet, V.I.

T. minor L. (Lesser Bladderwort.)

Locally distributed, Somenos Lake, V.I.

C. occilentalis A. Gray.

Locally distributed, Ccluelet, V.I.

U. vulgaris L. (Greater Bladderwort.)

Locally distributed, Prospect Lake, Y.I.; Shawnigan Lake. T.I.

\section{OROBANCHACEA (Broom-rape Family).}

Boschinakia (Poque).

F. Hookeri Walp.

Locally distributed, Yancouver Island-Alberni; Comox: Cameron Lake;

Nanoose; Cowichan; Shawnigan Lake; Mount Finlayson.

Orobaxcire (Broom-rape; Cancer-root).

O. comosa Hooli.

Locally distributed, southern half of Vancouver Island-Wellington;

Sidney; Victoria.

O. fasciculata Nutt. (Clustered Cancer-root.)

Rare. Menzies Creek: Cowichan, Glendemming: Observatory Ilill, V.I.

O. Iudoviciana Nutt. (Louisiana Broom-rape.)

Victoria, V.I.. ('ovele!).

O. pinorum Gey.

Rare. Cowichan River. V.I.. Glendemning.

O. uniflora L. (One-flowered Cancer lioot.)

Locally distributed. sonthern lalf of Vancourer Island-suroat Lake Falls,

Mrs. Cory Wood; Cowichan; Victoria. 


\section{PLANTAGINACE $\mathbb{E}$ (Plantain Family).}

Plantago (Plantain).

P. Bigelovii Gray.

I cocally distributed, southern end of Vancouver Island-Sidney; Victoria.

P. lanceolata L. (Rib Grass.)

Common. Vancouver Island.

P. macrocarja C. \& S.

Common. Vancourel Island; Queen Charlotte Islands.

P. major L. (Common Plantain.)

Common. Vanconver Island; Queen C'harlotte Islands.

var. minima Dene.

Wellington, V.I.; Victoria. V.I.

P. maritima I. (Seasile l'lantain.)

Common. Queen C'harlotte Islands; Vancouver Island.

\section{RUBIACEE (Madder Family).}

Galium (Bedistraw; Cleavers).

G. Aparine L. (Cleavers.)

Common. Vancouver Island.

G. Aparine var. Vaillantii Kóoll.

Port lienf rew, V.I.; Triangle Island, Ir. C. F. Tercombe.

G. boreale L. (Northern Redstraw.)

Generally distributed, Vancourer Island.

G. kamtschaticum (Stell.) var. oreganum Isitton. (Northern Wild Liquorice.) Locally distributed. Cowichan Lake, I.I.

G. trifidum L. (Small liedstraw.)

Common. Vancouver Island.

var. pacificum Wiegand.

Common. Tancouver Island.

G. triflorum Michx. (Sweet-scented Bedstraw.)

Common. Tancouver Island. Queen Charlotte Islands.

Silerardia (Spulrwort).

S. arensis I.

Locally distributed, Victoria, Sidney, V.I.

\section{CAPRIFOLIACEE (Honeysuckle Family).}

LINx.eA ('Twinflower).

L. botealis L. var. americana (Forbes) Rehter. (American Twinflower.)

Common. Vancourer Island; Queen rharlotte Islands.

Loviceri (Inneysuckle).

L. ciliosa (Pursh.) Poir. (Orange IIoneșuckle.)

Common. Vanconver Island.

I. lispidula Dougl. (Puple Honeysuckle.)

Locally distributed, southern half of Vancouver Islaud-Nanoose; Departure Bay; Malahat; Sidney.

L. iuroulcrata (Richalds) Banks. (Involncred Fly Honersuckle.)

Common. Queen ('harlotte Islands; Vancourer Island.

Sambets (Flder).

S. callical']n Greene. (Iied-fruited Elder.)

Common. Queen C'luarlotte Islands: Vancouver Island. 
SAMLCO'S- 'rutintied.

s. slatual Nutt. (Tree Elder: B]ue-fruited Elder.)

Locally distrihuted. soutleru lualf of Vancouver Is]and-Allerni ; Nanaino ; I) (111)аเи.

Srmplomicarpos (Snow Berry; Wax Rerry).

L. racemosus Miclix.

Common. Queen Charlotte Islands; Vancouver Is]and.

var. pauciflorus robbins.

Common. Vincourer Island.

Vheratal (Arrown-wood).

V. panciflorum Pylaie. (Few-flowered Cranberry Tipe.)

Locally distributed, Queen ('harlotte Islands (Osgood, "North American

Fauna," No. 21). Vaneonver Island-Cowirhan Ialke; IInderson Lake.

\section{VALERIANACEÆ (Valerian Family).}

Valeriaxa (Valerian).

I. septentrionalis Iisulb.

Henderson Lake. V.I., T. A. Tencrombr.

V. sitchensis Bong.

Generally distributed, Queen Charlotte Jslauds; Vanconver Island,

var. Scouleri (Rylb.) I'iper.

Locally distributed. Duncan, V.I.

Yalerianella (Colu Salad; Lambs Lettuce).

r. congesta LindI, (Sea Bunsh.)

Common. Vancouver Island.

T. Locusta (L.) Betcke.

Victoria. V.I.

V. samolitolia (Dr') Grisy.

Generally distributed, Queen Charlotte Islands. Vinconver IslandAlberui ; Salutlam.

\section{ECHINOCYSTIS.}

\section{CUCURBITACEAE (Gourd Family).}

E. oregana (Torr \& Gray) ('ogn. (Western Falsan Aplue.)

Rale. Saanich, Y.I., J. K. Androson: Hacoun.

Campanula.

\section{CAMPANULACE王 (Bluebell Family).}

C. Langsdorffiuna Fiscll.

Locally distributed, Queen ('har'lotte Islands. (Osgood, "North American Fauna," No. 21.)

C. Iasiocarya Cham.

Queen. Charlotte Islands. (Menry"s " Flora of Southern British Columbia.")

C. rotundifolia L. (IIarebell.)

Common. Vancouver Island.

Var. alaskana Gray. (Harebell.)

Tlell River, Queen Charlotte Islands, Dawson; Mount Mark, V.I., Mucum.

c. Scouleri Hook. (Sconler's Harebe]l.)

Common. Vaucouver Island. 
Specularia.

S. perfoliata (I.) A. DC. (Venus' Looking-glass.)

Rare. Southern end of Vancourer Island-Maple Bar; Victoria.

HETEROCONOX.

H. rariflorum Nutt.

Rare. Vicinity of Victoria, V.I., Couley.

\section{LOBELIACEA.}

LOBELIA.

L. Dortmanna L. (IVater Lobelia.)

Locally distributed, southern half of Vancouver Island-Sproat Lake; Cameron Lake; Shawnigan Lake.

\section{COMPOSIT死 (Composite Family).}

ACHILleA (Yarrow).

A. borealis Rons. (Torthern Yarrow.)

Locally distributed, Ycluelet. V.I.: Queen Charlotte Islands. (Osgood,

"North American Fauna," No. 21.)

1. millefolium L. (Milfoil ; Yarrow.)

Vancourer Island; Queen Charlotte Islands.

A. californica Pollard.

Rare. Saanich Arm, V.I., J. R. Anderson.

Adenocaulon (Silvel-green).

A. bicolor IIook.

Common throughout settled and cleared areas, Vancourer Island.

Agoseris (False Dandelion).

A. aurantiaca (Hook.) Gleene.

Locally distributed in mountains, Vancouver Island-Mount Arrowsmith.

A. glauca (Pursh.) Steud. (Large-flonered Fall Dandelion.)

Rare. (Mount Arrowsmith, V.I.), Fermode and Carter.

A. heterophylla (Nutt.) Greene.

Locally distributed, Vancourer Island-Victoria, Fletcher; Skirt Mount Sahtlam; Nanoose.

A. laciniata (Nutt.) Greene.

Tanconver Island-Alberni; Malahat; Sidney; Victoria.

rar. grandiflor'a Nutt.

Locally distributed, Vancouver Island-Cameron Lake; Qualicum; Nanaimo; Duncan.

A nibrosia (Ragweed).

A. trifida I. (Great Hawkweed.)

Locally distributed, Victoria, V.I.

Anaphalis (Pearly Everlasting).

1. margalitacea (I.) B. \& Ir. (Pearly or Large-flowered Everlasting.)

Common, Vancouver Island.

ras: occidentalis Greene.

Comnon. Vancouver Island.

val. subalpina (Gray) Iiydb.

Elk Ialke, V.I. 
AxTexxakia (Erelasting).

1. chlorintha Greene.

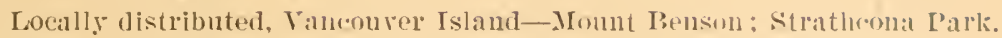

1. erigeroides Greene.

Locilly distributed, Iount Beuson, T.I.

1. eximia Greene.

Locally distributed, IIount Benson, Y.I.

1. Howellii Greene. (P'ussy's Toes.)

Common. Vinconvel Island.

1. medial Greene.

Lncally distributed, Mount Arrowsmith, V.I.

A. racemosa Hook.

Locally distributed, Stratlucona Park, V.I., I. M. M/uroum.

A. rosea Greene.

Rare. Monnt Arrowsmith, Y.I., Kermode and curter.

Axtrexis (chamomile).

1. arrensis L. (Field Chamomile.)

Common. Vancourer Island.

1. Cotula L. (May-weed; Fetid Chamomile.)

Common. Vancouver Island.

APARgIDIUM.

A. boreale (Boug.) T. \& G.

Locally distributed. Celuelet, V.I.; Queen Charlotte Islands and Bankis Island, $D r, C . F$. Neucombe.

Arctium (Burdock).

1. Lappa. I. (Great Burdock.)

Common. Vancouver Island.

A. minus Bernh. (Common Burdock.)

Common. Vancourer Island.

ARgEMIONE.

A. mexicana Linn.

Rare. Tictoria, J. R. Anderson.

ARNiCA.

A. amplexicaulis Nutt.

Tancouver Island-Mount Arrowsmith; Corrichan River.

A. aspera Greene.

Tancouver Island-Gordon River; Cowichan Lake.

A. betoniciefolia Greene.

Locally distributed, Cowichan Lake, V.I., J. R. Anderson; Henderson Lake.

1. Chamissonis Less.

Locally distributed, southern half of Tancouver Islaud-Comox; Kolsilah.

A. cordifolia Hook. (Heart-leaved Aruica.)

Rare. Mount Fimlasson, V.I.. Dr. C. F. Teucombe.

A. gracilis Rydb.

Mount Benson. Y.I., Hacomn.

A. latifolia Bong.

Common in mountains, Vancouver Island-Mount Alrowsmith: Stratheona

Park; Chemaiuns River; Cowichan Lake.

A. Macomii Greene.

Locally distributed, Comox. V.I., Macoun; Alberni Canal. Y.I. 
ARxica-c'ontinucel.

A. mollis Hook. (Hairy Arnica.)

Locally distributed, Yakoun Lake, Q.C.I., Dr. C. F. Texcombe; Alberni; Henderson Lake: Qualicum Falls; Quatsino. T.I.

Artemisia (Sagebrush; Wormwood).

A. Absinthilm L. (Common wormwood; Absinth.)

Locally distributed, Tancouver Island-Victoria; North Saanich.

A. canadensis Miclux. (Canada Wormwood.)

Locally distributed, Comox Spit, Y.I., Macoun; Victoria, T.I.

A. Indoviciana Nutt. (Dark-leaved Mugwort.)

Jocally distributed, Vanconver Island-Cowichan, Glendcnning; Departure Bas.

A. pracifica Nutt.

Victoria, T.I.

A. Sulkstorfii Piper.

Generally distributed, Vanconver Island.

A. rulgaris L. (Common Mugwort.)

Locally distributed, Victoria, Y.I.

Aster (Aster).

A. chilensis (yees.) DC:

Generi)lly distributed, Vancouver Island.

d. Cusickii Gray.

Locally distributed, Victoria, Y.I.

A. Donglasii Lindl.

Common. Vanconver Island.

A. Eatonii (Gray) Howell.

Locally distributerl, Elk Lake, T.I.

A. Engelmanui Gray.

Locilly distributed, Colwood, T.I.

A. foliaceus frondeus Gray. (Leafy-bracted Aster.)

Loc:ally alistributed, Yakom Lake, Queen Charlotte Islands, Dr. C. F.

Fencombe. Taucouver Island-cameron lake.

A. microlonchus Greene.

Locilly distributed, Victoria, V.I.

1. modestus Lindl. (Great Northeru Aster.)

Generally distributed, southern half of Vancouver Island-Albel'ui ; Wellington.

A. occidentalis Nutt.

Locally distributed, Namaino River, V.I., J.R. Anderson.

vir. intermedius Gray.

Locally distributed, Vancouver Island-Elk Lake.

A. oregatulus Nutt.

Locally distributed. Sidney, V.I., Macoun.

A. radulinus.

Ioc:ally distributed, Vanconver Island-Victoria; Horne Lake; Mount Finlilysun, Hucoum.

BERIA.

I;. maritima Gray.

- Barkley Sound; Solander Island; Triangle Island, Dr. C. F. Nexcombe.

Balsamolinza (Balsam Root).

B. Geltoikea Nutt.

Locally distributed, sonthern half of Tancouver Island-Cowichan; Cedar

IIill; V"ictoria, Macoun. Valdes Island, Dawson. 


\section{likLus (Daisy).}

B. percunis I. (Common Daiss.)

Common. Vanconver Island.

Bunks (Bur Marigold).

13. amplissima Greene.

Locally distributer, Vaneonver Island-Alberni.

Ii. Malcomili Greene.

Iocally distributed, southern half of Tancumver Island.

PIIUIA.

indeliu.

Experintental Farm, Siduey, V.I.

('AlENDULA.

C. urecusis $I_{\text {. }}$

Victoria, B.C.

('butaural (Star Thistle).

C. Calcitrupu L. (Star Thistle.)

I.ocally distributed, Vanconver Island-Nanaimo.

('. Cyunus L. (Curn Flower' ; Bachelor's Button.)

Locally distributed. Vanconver Island-Comox; Siduey; Cameron Lake. ('. Juceel L.

Locally distributed. Vanconver Island-Victoria.

(". melitensis J. (Tocilote.)

Locallỵ distributed, Vancouver Island-Nanaino; Escuimalt.

(. puiculutu $\mathrm{L}$.

locally distributed, Vanconver Island-Tictoria.

CHRYSANTHEMUM.

('. Leneduthrmum L. (Ox-ege Daiss.)

Common. Vaneouver Island.

r. Purthruium (I.) liernh.

Common. Vaucouver Istand.

('ichorilom (chicoly).

('. Intybus I. (('Inicoly; Blue Sailors.)

Generally distributed, Vancouver Island.

Constral (Thist](e).

(. arense (J.) Scop. (Canada Thistle.)

contuon. Vincouver Island.

('. edule Nitt. (Edible Thistle.)

Common. Vancourer Island; Queen Charlotte Islands. (Osgood, "North

American Fauma." No. 21.)

('. laurcolutum (L.) Scop. (Common Bur or Spear Thistle.)

Generally distributed, Tancouver Island.

C'OTULA (Mud Disk).

C. coronopifolia I.

Locally distributed, Vanconver Island-Nanaimo; Nanoose; Sidney.

('peepis (Halwlis-beard).

r. biemuis L. (Rough Hawk's-beard.)

Commun. Vancourer Island. 


\section{Creprs-Continued.}

C. capillaris (L.) Wallr. (Smooth Hawk's-beard.)

Common. Vancouver Island.

c. virens $\mathrm{L}$.

Chemanus and Cowichan, V.I., Macoun.

\section{Crocinfuar.}

C. multicaule Hook.

Iocally distributed, near Tictoria, V.I Macoun.

Frigron (Fleabane).

E. eamadensis I. (IIorse-weed.)

Common. Vancouver Island.

E. compositus Pursh.

Rare. Momut Arowsmith. T.I., Fumingliam.

F. phitadelphicus r. (Michaclmas Daisy.)

Common, Vancouver Islant.

F. peregrinus (I'ursh.) Greene.

Loeally distributed, Queen CIarlotte Islinds, Vancouver Island-MIount Arrowsmith, Mucoun.

E. ramosus septentrionalis (Felmald \& Wreg). (Daisy Fleabane.)

Locally distributed, Vanconvel Island-Alberni, Hacomn; Chemanus River.

E. salsuginosus (Richards) A. Gray.

Momnt Arrowsmith, V.I.; Chat ta Village, west roast, Q.C.I.

Friopitylum (Woolly sunflower).

E. C'usickii Eastman.

Salitlam, Y.I., E. M. Anrterson.

E. lanatum (Pursh.) Forbes.

(ommon. Vanconver Island. A rayless form plentifully collected in Victoria (Henry) may belong to this species.

E. lanatum var. Iencophylla $D($ :

Neal Victoria, Y.I. (Macoun and Fletcher). (Macomn's "Catalogue of Plants.")

EucepilaLus.

1). confinis.

I) ally distributed, Stratheona Park, V.I., J. W. Macomn.

N. Macomii Greene.

Locally distributed, Victoria, V.I., Mucrum.

Fraxslita (Samd-bur).

l. bipimatifida Nintt.

Locally distributed, Queen ('harlotte Islands, Damson. Yancourer Island - (ape Lazo) Victoria; isug Beach.

F. ('hamissonis (Toss.) $\mathrm{DC}$ '.

Jocally distributed on the ocean coast of Vuncourer lsland to Californla.

I'clnelet, Muromn.

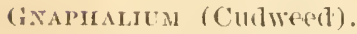

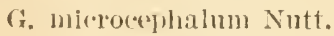

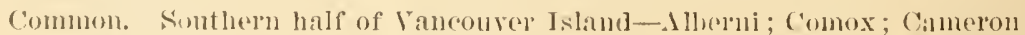

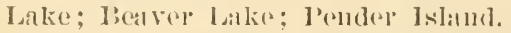

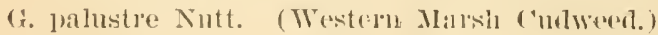

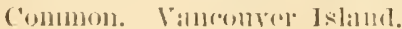


Gxaphalius-- C'ontinucl.

(i. lurlmlenm $\mathrm{I}$.

Common. Vimeotrer Island,

G. Sprengelii llook. \& Aru.

Victoria, V.I., I'letrher. (Macoun's " "alalogue of I'lants.")

G. uligrinosum (Ls) (Isw émberl; Waterwort.)

('ommon. Vancomver Islaunt.

Gimindelia (Gum-weel).

G. Anclesonii I'ij火'r.

Locally distributed, Sitanich In]ct, V.I., J. R. Luderson.

(i. collina J. K. Henry.

Vancouver Island. (IIenry"s "Flora of Somtlern IBritish Columbia.")

(x. integrifolia DC:

Gencrally distributed, soutlern half of Vaneouver Island.

(i. lanata Greene.

Locally distributed, Vanconver Island-Victoria.

G. nana Nutt.

Locally distributed, Vanconver Islind-Cowichan. (Gloudenning's List, 1918.)

G. oregana Gray. ? (i. strictal DC.

Gencrally listributed, sonthern half of Vanconver 1slaud. Our common form on the high seat-heathes. Masset, (..C'.I.

G. oregana Wilkesiana r'iper.

Queen Charlotte Islands, Osgood.

G. squarrosil (Pursh.) Duval. (Broad-leaved Gum-veed.)

Iare. A single specinen, Esquimalt of Namimo Railway, V.I. The identification of our Griuldelia is somewlat uncertain, but must remain as such until a revision of the Genus has been completed.

Helexiud (Sneezeweed).

H. antumnale I. (Swampl Sunflower.)

Iocally distributed, sonthern end of Vancourer Island-Victoria.

Helantiles (simflower).

II. Ma.rimiliani Schrad.

Locally distributer, Vinumver Island-Victoria, Macoun.

1I. petiotaris Nutt. (Prairie Sunflower.)

Locally distributed, Fancouver Islaud-Nanaimo, Macoun.

Hieraciua (Ilawkweed).

II. albiflorum Hook.

Common. Queen C'harlotte Islands; Vancouver Islaud.

II. canadense Michx. (Canada Hawkweed.)

Victoria, V.I., Jacoun.

H. gracile Hook. (. Ipine Hawkweed.)

Common in mountains. Vancourer Island.

H. Scouleri IIook.

Departure Bay, V.I. (Meehan). (Macoun's "Catalogue of Plants.")

H. triste Cham.

Dawson IIarbour, west coast, Queen Charlotte Islands, Dr. C. F. Newcombe.

HYтоснеRIS (Cat's-ear).

H glabra $\mathrm{L}$.

Common, Vancouver Islancl. 


\section{HYPOCHERIS-CONtinued.}

\section{If. radicata $\mathrm{L}$.}

Common. Vancourer Islant.

JAIMEA.

J. carmosu (Less.) Griy.

In salt milshes along the sea-shore Vanconver Island to Califormia. (Piper aud Beattic's "Flora of the Northwest Coalst.")

LACTLCA (Lettuce).

L. muratis $\mathrm{L}$.

Locally clistributed, Walugh Creek, V.I., J. R. Anderson; Shawnigan Lake, V.I.

L. scariola I. (Prickly Lettuce.)

Common. Vancouver Island.

L. spicata (Lam.) Hitch. (Tall Plue Lettuce.)

Fenerally distributed, southern halt of Vaucourer Island-Comox;

Alberni; Wellington; Elk Lake.

Ladipsana (Niplewort).

L. communis $\mathbf{I}$.

Locally distributerl, southern end of Vancouver lsland-Victoria; North Saanich.

Leontodon (Fall Dandelion; Hawkbit).

L. hastilis L. var. vulgatis Koch. (Common IIawkit.)

Generally distributed, Vanconver Island.

L. mudicaulis (L.) Banks. (Rough or Hairy Hawkbit.)

Generally distributed, Vancourer Island.

LUINA.

I. hypolenca Benth.

Generally distributed, southern half of Vincomer Island-Great Central

Lake; Koksilah; Sooke Rivel; Cameron Lake.

Matricaria (Pimeapple-weed).

M. suateolens (P'ush.) Buchanan.

Generally distributed, Qmeen ('luarlotte Islands; Vancouver Island.

Madia ('Tallweed).

I. dissiliflora (Nutt.) T. \& G.

Locally distributed, Sidney, V.I., Macomu.

M. exigua Smith.

Generally distributed, southern half of Vancouvep Island-Alberni;

Victoria.

M. glomerata llook.

Generally distributer, southern half of Vancouver Island.

M. madioides Nitt.

Common. Southern half of Vancourer Island.

M. racemosa (Nutt.) 'T. \& (t.

Common. Southern halt of Vancouver Island.

M. sativa Mol.

Generally distribufed, southern half of Vancouver Island-Alberni to Victoria. 
MITROSERIS.

M. Bigelovii Graty.

Locally distributed, soutlern half of Vancouver Island-Ninoose; Victoria.

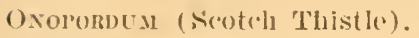

\section{U. Acullhimu I.}

Locally distributed, Vancouver Island-Nanaimo; Cowichan River.

I'ETAsites (Coltsfoot; Futterbur).

P. frifrida (I.) Fries. (Mpine Coltsfoot.)

Locally distributed in the mountains, Vancouver Island-Mount Arrowsmith.

I'. sperciosil (Nutt.) Piprer. (Palmate-leaf Sweet Coltsfoot; Butterbur.)

Commen. Vancouver Island.

Prexantmas (liattlesulie Root).

P. hastatal (Less.) A. Gray.

Common. Queen Charlotte Islands; Vanconver Island.

Psilocarpilis.

P. elatior Gray.

Victoria, V.I.; Sidney, V.I., ILcoun.

P. oreganus Nutt:

Samichton. V.I., Mucoun.

P. tenellus Nutt.

Victoria, V.I.; Deel Cove, V.I., Macoun.

liUdeEckia (C'one-flower).

R. hiva L. (Blackeyed Śusan; Yellow Daisy.)

Locally distributed, Comox, V.I.; Cowichan. V.I.

SAUSSUREA (Saw-wolt).

S. densa (Hook.) Rydb.

Locally distributed, Mount Bemson, V.I.

Senecio (Groundsel; Ragwort).

S. Balsamitie Muhl.

Iocally distributed. Vancouver Island-Comox, north, on the east side of the Island.

S. fastigiatus Nutt. rar. Maromii (rreene.) Greenman.

Generally distributed, Vancouver Island, chiefly on the enst side.

S. Newcombei Greene.

Locally distributed, West Coast, Queen Clualotte Islands, Dr. C. $F$. Neucombe.

S. Pseudo-Arnica Less. (Sea-beach Senecio.)

I ocally distributed, Vancouver Island; Queen Charlotte Islands, Dauson.

S. resedifolius Less.

Locally distributed, Queen Cliarlotte Islands. (Osgood, "North American Fauna," No. 21.)

S. sulvaticus L. (Tood Groundsel.)

Commoin. Vancouver Island.

S. triangularis Hook.

Locally distributed, Vancouver Island-Mount Arrowsmith; Chemainus liver.

S. vutratis I. (('ommon Groundsel.)

Common. Vilncouver Island. 
SERICocarpl's (White-toljed Aster).

S. rigidus Lind].

Locally distributen, Vancouver Island--Wellington; Cowichan; Victoria.

Silybun (Milk Tlistle).

S. marianmm (L.) Gaertu.

Locally distributed, Nanaimo, Y.I.

Solidago (Goldell-lod).

s. glutinosi Nintt.

Locally distributed, Vanconver Island-Comox, Macoun; Aberni.

S. humilis Pursh.

Iocally distribnted, Momt Penson, V.I., J. R. Anterson.

S. Iepitta $\mathrm{DC}$.

Common. Vancouver Island; Skidegate, Queen Charlotte Islands.

vas. elonsata (Nutt.) Fernald.

Common. Vincourel' Islanel.

S. Sillebrosa Piper.

Oak Bay, Victoria, V.I., Di. C. F. Nexcombe.

Soverus (Sow Thistle).

S. arrensis L. (Ammal Sow Thistle.)

Common. Vanconvel Islant.

s. asper (L.) Hill. (Spiny Sow Thistle.)

Common. Vanconver Island.

s. ulcracens J. (Halle's Lettace.)

Common. Vancourer Island.

S'TEPHANOMERIA.

S. minor Nutt.

The only record. Camplell River, V.I., J. R. Anderson.

TANACETUM ('Tansy).

T. huroneuse Nutt. (Lalke JImron Tausy.)

Locilly distributerl, Qneen Charlotte Islands, Dauson. Vanconver IslandRenfrew Iistrict; Ueluelet; Clayoquot; Nootka.

T. inlyare $\mathrm{I}$.

Gencrally distribated, Vincouver Island.

Taraxactam (Dandelion).

T. officinule Weber.

Common. Vancourer Island.

Tragorogon (Salsify ; Oystel Plant).

T. porrifolius I. (Purple Oyster Plant.)

Locally tistributed, Vancouver Island-Victoria; Cowichan.

\section{Xanthium (Cocklebur).}

X. spinosum (Spiny Burweed.)

Rare. Vancouver Island-Namaimo, Macomn. 


\section{ADDENDA.}

Oil liane 9 .

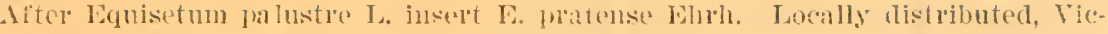
turia, Alberni, c'amerom l,ike, V.I.

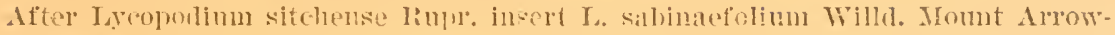
sulll, Y.1., Jaromn.

On page 11.

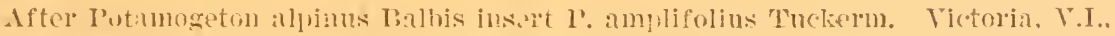
J/(เ) un.

On page 13.

Insert Agrostis exarata Trin. in place of $\Lambda$. exarata $x$ alloa.

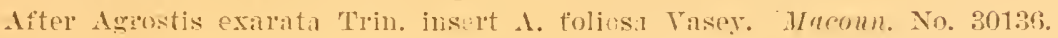

Onit Agrostis infata Shribner, whirh is a spnonym of A. glumerata (Presl.) Fimth.

Insert Arrostis Rosse Vasep for A. Vilinins Trin.

After Acrostis stolnifera r. omit vilr. maritima.

Cmit Agrostis rulgaris With.. a variety of A. alloa $I_{\text {. }}$.

Alopecurus aristulatus Torr.. a variety of $A$. geniculatis.

On page 14 .

Rromus commutatus Selnrad. introduced.

After Bromus eximius (Slear.) I'ipej insert B. Hookeriants 'Thulber. Gencrally distributed, sonthern half of Vancouver Islant.

On page 20.

After Polypogon littoralis (With) Smith insert P. Monspeliensis (L.) Macf. Iracomil, both of which are introduced.

On page 23.

Omit Carex leiocarpa C. 1. Meyer, a symonym of $\mathrm{C}$. anthoxantha Presl.

On page 2 ?f.

Omit Juncus nodosus I.

On page 30 .

Cerastimm risensum introduced.

On lage 42.

Omit Brassica sinapistrmm Toiss. a synnym of R. arrensis (LA.) Titze.

On lage 46 .

After Sedum stenopetalum Pursu, onit Qheen C'harlotte Islanris.

On page 49

Potentilla albida Rydi). introduced.

On page .14.

Vicia lathroides L. introdnced.

On page 55.

After Euplorbia hirsuta (Torr.) Wiegand insert E. Latlyrus L. Victoria. V.I. 
On page 57.

Elodea campanulata Pursh. is Irpericum viroinicum of Gray"s List.

On page 62.

Osmorrhiza brevipes (C. \& R.) Suksilorf.

On page 69.

Omit Allocarsa californica (Dr.) Treene; not credited to Washington or British Columbia by Professor C. T. Piper.

On page 71.

Omit Mentha viridis L., a synonym of M. spicata L.

On page $7 \pi$.

Omit Valeriana septentrionalis Rydb.

On page $s 0$.

After Artenisia ludoviciana Nutt. insert A. occidentalis Nutt. Victoria, V.I.

On page 84 .

After Madia sativa Mol. insert M. sativa capitata (Nutt.) Piper. Piner \& Beattie's "Flora of the Northwest Coast"; probably our common form referred by us to M. sativa Mol.

In conclusion, we are largely indebted to Plofessor C. Y. Piper, of Washington, D.C., for the identification of many of our plants which would not otherwise have appeared in this List. 


\section{INDEX TO FAMILIES.}

\begin{tabular}{|c|c|}
\hline Aceraces $\ldots \ldots \ldots \ldots \ldots \ldots \ldots \ldots .56$ & I. (2)unilacea \\
\hline Alismacea $\ldots \ldots \ldots \ldots \ldots \ldots \ldots \ldots$ 1: & Lentibulariacea .............. T5 \\
\hline maranthaceas $\ldots \ldots \ldots \ldots \ldots \ldots \ldots$. & Liliaces $\ldots \ldots \ldots \ldots \ldots \ldots \ldots$ \\
\hline nacardinacea $\ldots \ldots \ldots \ldots \ldots \ldots \ldots$. & Limnanthacere $\ldots \ldots \ldots \ldots \ldots \ldots$ \\
\hline 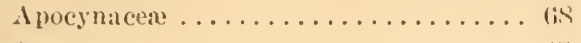 & Iinacex $\ldots \ldots \ldots \ldots \ldots \ldots \ldots \ldots$ \\
\hline raced $\ldots \ldots \ldots \ldots \ldots \ldots \ldots \ldots \ldots \ldots$ & Iobeliacese $\ldots \ldots \ldots \ldots \ldots \ldots \ldots$ \\
\hline ralistcer $\ldots \ldots \ldots \ldots \ldots \ldots \ldots \ldots$ & Iomanthacese $\ldots \ldots \ldots \ldots \ldots \ldots \ldots$.32 \\
\hline ristolochiaceat $\ldots \ldots \ldots \ldots \ldots \ldots \ldots$ & Lscopodiacear $\ldots \ldots \ldots \ldots \ldots \ldots \ldots$ \\
\hline rerberidacea $\ldots \ldots \ldots \ldots \ldots \ldots \ldots \ldots$ & Iythracex $\ldots \ldots \ldots \ldots \ldots \ldots \ldots$ \\
\hline etulacea $\ldots \ldots \ldots \ldots \ldots \ldots \ldots . .$. & Malvacese $\ldots \ldots \ldots \ldots \ldots \ldots \ldots \ldots$ \\
\hline oraginacese $\ldots \ldots \ldots \ldots \ldots \ldots \ldots \ldots$ & Myricaces $\ldots \ldots \ldots \ldots \ldots \ldots \ldots$ \\
\hline Cactacex $\ldots \ldots \ldots \ldots \ldots \ldots \ldots \ldots$ & Najadacese $\ldots \ldots \ldots \ldots \ldots \ldots \ldots \ldots$ \\
\hline Callitrichacex $\ldots \ldots \ldots \ldots \ldots \ldots \ldots$ & inacese.$\ldots \ldots \ldots \ldots \ldots \ldots \ldots$ \\
\hline Campanulacea $\quad \ldots \ldots \ldots \ldots \ldots \ldots \ldots$ & Nymphreacere ................... 39 \\
\hline ('ip)rifoliacese $\ldots \ldots \ldots \ldots \ldots \ldots \ldots$ & Onagracese $\ldots \ldots \ldots \ldots \ldots \ldots \ldots$ \\
\hline phyllacex $\ldots \ldots \ldots \ldots \ldots \ldots \ldots$ & ssacere $\ldots \ldots \ldots \ldots \ldots \ldots$ \\
\hline Celastracese $\ldots \ldots \ldots \ldots \ldots \ldots \ldots \ldots$ & $\ldots \ldots \ldots \ldots \ldots \ldots$ \\
\hline acese $\ldots \ldots \ldots \ldots \ldots \ldots$ & асељ..$\ldots \ldots \ldots \ldots$ \\
\hline Chenopodiaces $\ldots \ldots \ldots \ldots \ldots \ldots \ldots$. & $\ldots \ldots \ldots \ldots \ldots \ldots$ \\
\hline itse $\ldots \ldots \ldots \ldots \ldots \ldots \ldots \ldots \ldots$ & ex $\ldots \ldots \ldots \ldots \ldots \ldots \ldots, 41$ \\
\hline acere $\ldots \ldots \ldots \ldots \ldots \ldots \ldots$ & $x \ldots \ldots \ldots \ldots \ldots \ldots \ldots \ldots \ldots \ldots \ldots$ \\
\hline cor'uacee $\ldots \ldots \ldots \ldots \ldots \ldots \ldots \ldots$ 6.; & inacese $\ldots \ldots \ldots \ldots \ldots \ldots \ldots$ \\
\hline cee $\ldots \ldots \ldots \ldots \ldots \ldots \ldots \ldots$ & Plumbaginace $\ldots \ldots \ldots \ldots \ldots \ldots \ldots$. 66 \\
\hline Crucifere $\ldots \ldots \ldots \ldots \ldots \ldots \ldots \ldots$ & сея $\ldots \ldots \ldots \ldots \ldots \ldots 6$. \\
\hline ссея $\ldots \ldots \ldots \ldots \ldots \ldots \ldots$. & Polsgonacex $\ldots \ldots \ldots \ldots \ldots . . . . . .39$ \\
\hline cea $\ldots \ldots \ldots \ldots \ldots \ldots \ldots \ldots$. & odiaceæ $\ldots \ldots \ldots \ldots \ldots \ldots \ldots \ldots$ \\
\hline Droseracere $\ldots \ldots \ldots \ldots \ldots \ldots \ldots \ldots, 45$ & ere $\ldots \ldots \ldots \ldots \ldots \ldots \ldots$ \\
\hline cere $\ldots \ldots \ldots \ldots \ldots \ldots \ldots \ldots$ & Primulace $\ldots \ldots \ldots \ldots \ldots \ldots \ldots$ \\
\hline Elintinacese $\ldots \ldots \ldots \ldots \ldots \ldots \ldots \ldots .57$ & ceea $\ldots \ldots \ldots \ldots \ldots \ldots \ldots$ \\
\hline Empetraces $\ldots \ldots \ldots \ldots \ldots \ldots \ldots \ldots$ & $\operatorname{cex} \ldots \ldots \ldots \ldots \ldots \ldots \ldots$ \\
\hline Equisetacea $\ldots \ldots \ldots \ldots \ldots \ldots \ldots \ldots$ & Rosacere ......... \\
\hline 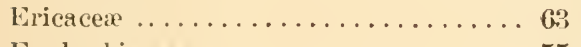 & cer $\ldots \ldots \ldots \ldots \ldots \ldots \ldots \ldots$ \\
\hline Auphorbiacese .................. & Salicaceæ $\ldots \ldots \ldots \ldots \ldots \ldots \ldots$ \\
\hline$\ldots \ldots \ldots \ldots$ & lacex $\ldots \ldots \ldots \ldots \ldots \ldots \ldots$. 32 \\
\hline riacese $\ldots \ldots \ldots \ldots \ldots \ldots \ldots \ldots+1$ & 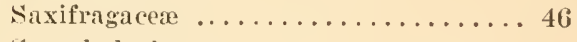 \\
\hline Gentiauacere $\ldots \ldots \ldots \ldots \ldots \ldots \ldots \ldots 6$. 6 . & Scrophulariaced $\ldots \ldots \ldots \ldots \ldots$ \\
\hline Geraniaces $\ldots \ldots \ldots \ldots \ldots \ldots \ldots \ldots .65$ & Selaginellacese $\ldots \ldots \ldots \ldots \ldots \ldots \ldots$ \\
\hline Graminer $\ldots \ldots \ldots \ldots \ldots \ldots \ldots \ldots \ldots, 12$ & ${ }^{\prime} \ldots \ldots \ldots \ldots \ldots \ldots \ldots$ \\
\hline Haloragidacese $\ldots \ldots \ldots \ldots \ldots \ldots \ldots, 60$ & Sparganiacene $\ldots \ldots \ldots \ldots \ldots \ldots \ldots$ \\
\hline Hydrophyllacex $\ldots \ldots \ldots \ldots \ldots$ & Taxacese ................... \\
\hline Hspericacet $\ldots \ldots \ldots \ldots \ldots \ldots \ldots .57$ & Typhacese ................. \\
\hline Iridacea $\ldots \ldots \ldots \ldots \ldots \ldots \ldots \ldots \ldots \ldots$ & Umbelliferse $\ldots \ldots \ldots \ldots \ldots \ldots \ldots$. 60 \\
\hline 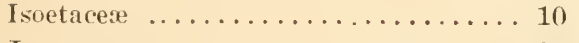 & Urticaceæ $\ldots \ldots \ldots \ldots \ldots \ldots \ldots \ldots . \ldots . \ldots$ \\
\hline Juncice:e $\ldots \ldots \ldots \ldots \ldots \ldots \ldots \ldots .25$ & Valerianacex $\ldots \ldots \ldots \ldots \ldots \ldots \ldots$ it \\
\hline Juncaginacer $\quad \ldots \ldots \ldots \ldots \ldots \ldots \ldots$ 12 & aасељ...$\ldots \ldots \ldots \ldots \ldots \ldots$ \\
\hline$\ldots \ldots \ldots \ldots$ & Violacey $\ldots . . . \ldots \ldots \ldots \ldots . . . \ldots$ \\
\hline & \\
\hline
\end{tabular}








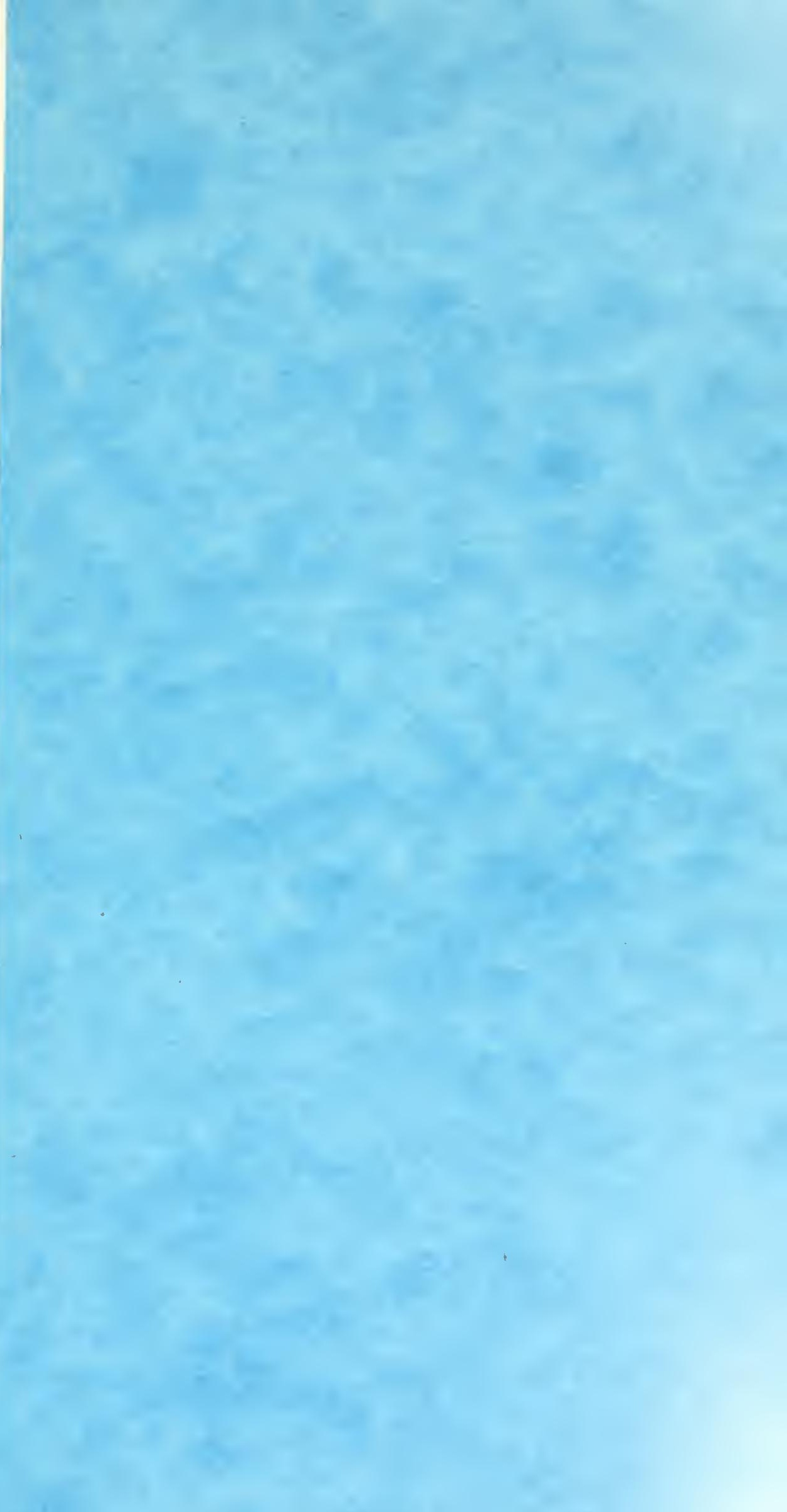


